\title{
Decaimento dos autovalores de operadores integrais positivos gerados por núcleos Laplace-Beltrami Diferenciáveis
}





\title{
Decaimento dos autovalores de operadores integrais positivos gerados por núcleos Laplace-Beltrami diferenciáveis
}

\author{
Mario Henrique de Castro
}

Orientador: Prof. Dr. Valdir Antonio Menegatto

Co-orientadora: Profa. Dra. Ana Paula Peron

Tese apresentada ao Instituto de Ciências Matemáticas e de Computação - ICMC-USP, como parte dos requisitos para obtenção do título de Doutor em Ciências - Matemática . VERSÃO REVISADA

USP - São Carlos

Agosto de 2011 
Ficha catalográfica elaborada pela Biblioteca Prof. Achille Bassi e Seção Técnica de Informática, ICMC/USP, com os dados fornecidos pelo(a) autor(a)

de Castro, Mario Henrique
Decaimento dos autovalores de operadores
integrais positivos gerados por núcleos Laplace-
Beltrami diferenciáveis / Mario Henrique de Castro;
orientador Valdir Antonio Menegatto -- São Carlos,
2011. 68.
p.
Tese (Doutorado - Programa de Pós-Graduação em
Matemática)-- Instituto de Ciências Matemáticas e
de Computação, Universidade de São Paulo, 2011.
1. Decaimento de autovalores. 2. Operadores
integrais. 3. Derivação termo a termo. 4. Teoria de
Mercer. I. Menegatto, Valdir Antonio, orient. II.
Título.


Aos meus pais, Carlos e Madalena. 



\section{Agradecimentos}

Apesar da tese levar somente o meu nome na autoria, ela não estaria pronta sem a ajuda, direta ou indireta, de várias pessoas. Desta forma, expresso minha gratidão ao meu orientador, Prof. Dr. Valdir A. Menegatto, e à minha co-orientadora, Profa. Dra. Ana Paula Peron, pela orientação e dedicação; aos meus pais, Carlos e Madalena, e irmãos, Aline e Fernando, que sempre acreditaram no meu potencial acadêmico e me incentivaram a continuar meus estudos; aos meus amigos, pelo companheirismo e lealdade; aos professores e funcionários do ICMC-USP que me ajudaram no ato de suas obrigações trabalhistas, e principalmente aos que o fizeram por prazer; aos colegas de trabalho da FAMAT-UFU, pelo incentivo; e à CAPES, pelo apoio financeiro de agosto de 2008 a janeiro de 2011. 

Neste trabalho obtemos taxas de decaimento para autovalores e valores singulares de operadores integrais gerados por núcleos de quadrado integrável sobre a esfera unitária em $\mathbb{R}^{m+1}, m \geq 2$, sob hipóteses sobre ambos, certas derivadas do núcleo e o operador integral gerado por tais derivadas. Este tipo de problema é comum na literatura, mas as hipóteses geralmente são definidas via diferenciação usual em $\mathbb{R}^{m+1}$. Aqui, as hipóteses são todas definidas via derivada de Laplace-Beltrami, um conceito genuinamente esférico investigado primeiramente por W. Rudin no começo dos anos 50. As taxas de decaimento apresentadas são ótimas e dependem da dimensão $m$ e da ordem de diferenciabilidade usada para definir as condições de suavidade. 

In this work we obtain decay rates for singular values and eigenvalues of integral operators generated by square integrable kernels on the unit sphere in $\mathbb{R}^{m+1}, m \geq 2$, under assumptions on both, certain derivatives of the kernel and the integral operators generated by such derivatives. This type of problem is common in the literature but the assumptions are usually defined via standard differentiation in $\mathbb{R}^{m+1}$. Here, the assumptions are all defined via the Laplace-Beltrami derivative, a concept first investigated by $\mathrm{W}$. Rudin in the early fifties and genuinely spherical in nature. The rates we present are optimal and depend on both, the differentiability order used to define the smoothness conditions and the dimension $m$. 



\section{Sumário}

Introdução $\quad$ Xv

\begin{tabular}{lll}
\hline & Preliminares & 1
\end{tabular}

1.1 Topologia e análise . . . . . . . . . . . . . . . . . . . . . . . . . . . 1

1.2 Teoria da medida $\ldots \ldots \ldots \ldots$. . . . . . . . . . . . . . 3

1.3 Análise funcional . . . . . . . . . . . . . . . . . . . . . 5

1.4 Os harmônicos esféricos . . . . . . . . . . . . . . . . . . . . . . . . . . . 13

1.5 O operador de Laplace-Beltrami . . . . . . . . . . . . . . . . . . . . . . 15

1.6 A derivada de Laplace-Beltrami . . . . . . . . . . . . . . . . . . . . . 17

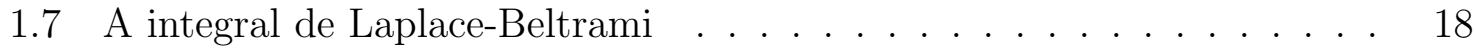

2 Teoria de Mercer em espaços topológicos 21

$2.1 \quad$ Representação em forma de série para os núcleos geradores . . . . . . . 21

2.2 Núcleos geradores suaves $\ldots \ldots \ldots$. . . . . . . . . . . . . . . . . . . 28

2.3 Nuclearidade . . . . . . . . . . . . . . . . . . . . . . . . . . . . . 31

3 Núcleos Laplace-Beltrami diferenciáveis 33

3.1 Positividade e a derivada de Laplace-Beltrami . . . . . . . . . . . . . . 35

3.2 Derivação termo a termo . . . . . . . . . . . . . . . . . . . . 39

4 Decaimento de autovalores

4.1 Resultados principais . . . . . . . . . . . . . . . . . . . . . . . . 43

4.2 Otimalidade dos resultados . . . . . . . . . . . . . . . . . . . . . . 51

4.3 Uma família de exemplos . . . . . . . . . . . . . . . . . . . . 56

\begin{tabular}{ll}
\hline Referências Bibliográficas & 59
\end{tabular}

$\begin{array}{ll}\text { Índice Remissivo } & 67\end{array}$ 

Sejam $m$ um inteiro positivo maior ou igual a 2 e $S^{m}$ a esfera unitária em $\mathbb{R}^{m+1}$ munida com a medida de Lebesgue $\sigma_{m}$. Neste trabalho consideramos operadores integrais definidos pela expressão

$$
\mathcal{K}(f)=\int_{S^{m}} K(\cdot, y) f(y) d \sigma_{m}(y)
$$

onde o núcleo gerador $K: S^{m} \times S^{m} \rightarrow \mathbb{C}$ é um elemento de $L^{2}\left(S^{m} \times S^{m}, \sigma_{m} \times \sigma_{m}\right)$. Neste caso, o operador obtido é compacto sobre $L^{2}\left(S^{m}, \sigma_{m}\right)$. Para simplificar a notação, denotamos os espaços recém mencionados por $L^{2}\left(S^{m} \times S^{m}\right)$ e $L^{2}\left(S^{m}\right)$, respectivamente.

Se $K$ é positivo definido no sentido de que

$$
\int_{S^{m}} \int_{S^{m}} K(x, y) f(x) \overline{f(y)} d \sigma_{m}(x) d \sigma_{m}(y) \geq 0, \quad f \in L^{2}\left(S^{m}\right)
$$

então $\mathcal{K}$ também é autoadjunto e o teorema espectral para operadores compactos e autoadjuntos (ou Teorema de Hilbert-Schmidt) é aplicável e garante que

$$
\mathcal{K}(f)=\sum_{n=0}^{\infty} \lambda_{n}(\mathcal{K})\left\langle f, f_{n}\right\rangle_{2} f_{n}, \quad f \in L^{2}\left(S^{m}\right),
$$

onde $\left\{\lambda_{n}(\mathcal{K})\right\}$ é uma sequência de números reais não negativos (possivelmente finita) decrescendo para 0 e $\left\{f_{n}\right\}$ é uma base ortonormal de $L^{2}\left(S^{m}\right)$. Os números $\lambda_{n}(\mathcal{K})$ são os autovalores de $\mathcal{K}$ e a sequência $\left\{\lambda_{n}(\mathcal{K})\right\}$ leva em consideração possíveis repetições impostas pela multiplicidade algébrica de cada autovalor. A ortogonalidade mencionada se refere ao produto interno usual $\langle\cdot, \cdot\rangle_{2}$ de $L^{2}\left(S^{m}\right)$. A positividade definida de $K$ significa nada mais que a positividade do operador integral $\mathcal{K}$. Por estar relacionada com o produto interno de $L^{2}\left(S^{m}\right)$, esta propriedade costuma ser chamada de $L^{2}$ - positividade definida. 
Adiantamos que a adição de continuidade do núcleo $K$ ao contexto implica que $\mathcal{K}$ é um operador nuclear (veja o Teorema 2.3.1), isto é,

$$
\sum_{f \in B}\left\langle\mathcal{K}^{*} \mathcal{K}(f), f\right\rangle_{2}^{1 / 2}<\infty
$$

qualquer que seja a base ortonormal $B$ de $L^{2}\left(S^{m}\right)$. Em particular,

$$
\sum_{n=1}^{\infty} \lambda_{n}(\mathcal{K})<\infty,
$$

e podemos extrair a mais elementar taxa de decaimento para autovalores de tais operadores, $\lambda_{n}(\mathcal{K})=o\left(n^{-1}\right)$, ou seja, $\lim _{n \rightarrow \infty} n \lambda_{n}(\mathcal{K})=0$.

Se o operador compacto não se encaixa no contexto do teorema espectral (o caso mais comum ocorre quando o operador não é autoadjunto), o foco das atenções passa a ser a sequência dos valores singulares do operador. Se $T$ é um operador compacto sobre $L^{2}\left(S^{m}\right)$, então o operador $|T|:=\left(T^{*} T\right)^{1 / 2}$ é compacto, positivo e autoadjunto sobre o mesmo espaço. Por definição, os valores singulares de $T$, aqui denotados por $s_{n}(T)$, são os autovalores de $|T|$. Claramente, a sequência $\left\{s_{n}(T)\right\}$ pode ser ordenada de forma decrescente, incluindo repetições de acordo com sua multiplicidade algébrica como autovalores de $|T|$. Desta forma, a caracterização de nuclearidade de um operador compacto e positivo $T$ sobre $L^{2}\left(S^{m}\right)$ se reduz a

$$
\sum_{n=1}^{\infty} s_{n}(T)<\infty
$$

e, quando este for o caso, obtemos como antes $s_{n}(\mathcal{K})=o\left(n^{-1}\right)$.

O estudo do comportamento assintótico de autovalores e valores singulares de operadores é um problema clássico. A primeira menção de estimativas de autovalores parece ter sido feita no início do século XX por I. Fredholm em [32]. Ainda na primeira metade do século passado, Hille e Tamarkin ([40]), Gel'fond ([33]) e Weyl ([85]) deram importantes contribuições para a teoria, que depois também seria abordada por Grothendieck, Gohberg e Krein, e König em [37], 34] e [43], respectivamente. No começo, o problema consistia em obter condições sobre um operador $T$ que garantissem que a sequência $\left\{\lambda_{n}(T)\right\}$ de autovalores pertencesse a algum subconjunto de $c_{0}$. No contexto dos operadores integrais, tais condições foram dadas em termos de propriedades de integrabilidade e diferenciabilidade do núcleo gerador. Resumindo, o objetivo era provar que quanto mais suave o núcleo, mais rápida a convergência de $\left\{\lambda_{n}(T)\right\}$.

$\mathrm{O}$ caso em que o núcleo gerador $K$ é uma função definida no produto cartesiano de dois intervalos compactos (em geral $[0,1] \times[0,1])$ já está consolidado. Além das referências clássicas do parágrafo anterior, também gostaríamos de citar [19, 38, 39, 
44, 62, 64, 65, 69, 70, 83. Os casos em que os intervalos são substituídos pela reta real ou subconjuntos abertos de espaços euclidianos foram estudados por Buescu e Paixão em [9], e Menegatto e Ferreira em [28]. Resultados da mesma natureza podem ser encontrados em muitas outras referências e não necessariamente no contexto discutido aqui. Em particular, mencionamos o uso de hipóteses envolvendo a continuidade de Hölder sobre $K$ em [18, 45, 46]. Recentemente, V. Menegatto e colaboradores obtiveram êxito na análise do decaimento dos autovalores de operadores integrais gerados por núcleos $K: S^{m} \times S^{m} \rightarrow \mathbb{C}$ assumindo condições de suavidade do tipo Lipschitz sobre as derivadas usuais de $K([30])$.

No Capítulo 4 investigamos a mesma questão analisada nestas referências, mas utilizando diferenciabilidade no sentido de Laplace-Beltrami para definir a hipótese básica de suavidade para o núcleo gerador. Além disso, também mostramos que as taxas de decaimento obtidas são as melhores possíveis (sobre o método de verificação da otimalidade do decaimento, recomendamos a leitura de [71, 72]). Pelo que sabemos, esta abordagem é nova e mais apropriada visto que tal noção de diferenciabilidade é um conceito genuinamente esférico, apresentando muitas propriedades interessantes e aplicações em conexão com Teoria da Aproximação (veja [2, 86, 87] e as referências lá contidas). A propósito, a derivada de Laplace-Beltrami foi introduzida por Rudin em [75] e depois desenvolvida por Wherens em [86, 87], mas somente para o caso $m=2$. O caso geral é amplamente discutido em [54, 66].

O Capítulo2 é reservado ao estudo de representações em série para núcleos positivos definidos. Resultados deste tipo encaixam-se no que denominamos Teoria de Mercer, em homenagem ao matemático inglês James Mercer que, em 1909, publicou seu famoso artigo entitulado "Functions of positive and negative type and their connection with the theory of integral equations" contendo o seguinte resultado, hoje conhecido como Teorema de Mercer:

Teorema de Mercer [55]. Todo núcleo positivo definido $K:[0,1] \times[0,1] \rightarrow \mathbb{R}$, contínuo e simétrico, possui uma representação em série da forma

$$
K(x, y)=\sum_{n=1}^{\infty} \lambda_{n}(\mathcal{K}) \phi_{n}(x) \overline{\phi_{n}(y)}, \quad x, y \in[0,1],
$$

onde $\left\{\lambda_{n}(\mathcal{K})\right\}$ é uma sequência de números reais não negativos convergente para 0 e $\left\{\phi_{n}\right\}$ é um conjunto ortonormal em $L^{2}([0,1])$, formado por funções contínuas.

Muitas versões do Teorema de Mercer surgiram desde a publicação de sua versão original. Algumas destas generalizações e aplicações do Teorema de Mercer podem ser encontradas em [4, 5, 10, 12, 26, 29, 42, 59, 60, 68, 77, 81, 82]. Apresentamos aqui uma versão do teorema para o caso em que $X$ é um espaço topológico primeiro-enumerável e 
localmente compacto, algo que não encontramos na literatura e que abrange, com folga, nosso contexto esférico. Este resultado gera uma forma mais simples para a verificação da nuclearidade de operadores integrais já que ele determina uma relação entre o traço do operador e uma integral envolvendo exclusivamente o núcleo do operador (veja o Teorema 2.3.1).

No Capítulo 3, voltamos ao ambiente esférico tratando da ação da derivada de Laplace-Beltrami sobre núcleos suficientemente suaves - aqueles definidos por expansões absolutamente e uniformemente convergentes geradas por famílias de funções que são ao menos contínuas (como no Teorema de Mercer). Os principais resultados deste capítulo descrevem condições que tornam possível a troca da derivada de LaplaceBeltrami com o somatório da série que representa o núcleo e apresentam condições necessárias para que certas derivadas de núcleos positivos definidos também definam núcleos positivos definidos. Mais uma vez, a importância destes resultados está no fato de oferecerem condições e ferramentas para que verifiquemos se determinados núcleos satisfazem, ou não, as hipóteses dos nossos principais resultados quando procuramos por exemplos.

Resultados conhecidos utilizados no decorrer do texto são enunciados no Capítulo 1 juntamente com as referências de origem. 


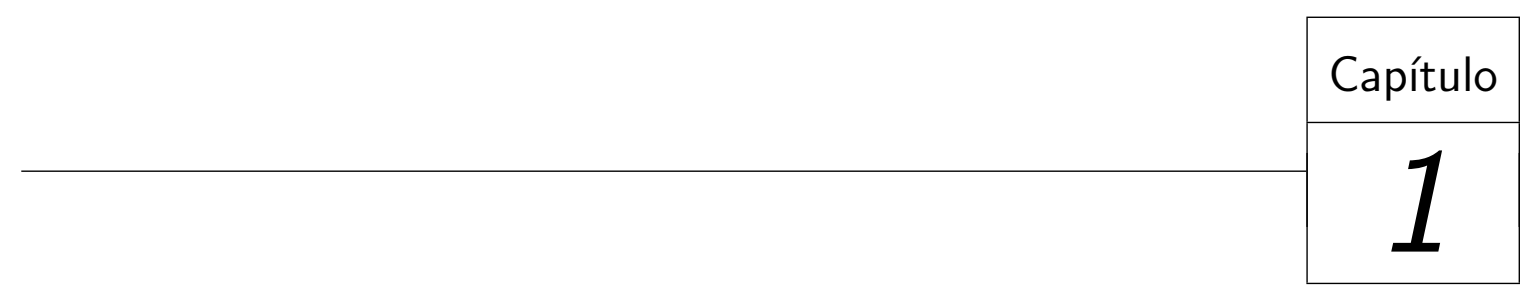

Preliminares

Neste capítulo apresentamos conceitos matemáticos básicos e resultados já conhecidos utilizados no decorrer da tese. Sempre que possível oferecemos uma referência para o resultado. O leitor interessado nos resultados inéditos da tese pode optar pela omissão deste capítulo, indo diretamente para o próximo.

\subsection{Topologia e análise}

Começamos relembrando alguns conceitos topológicos que utilizamos no estudo da Teoria de Mercer e vários resultados relacionados com a convergência de séries e sequências de números ou funções. A referência básica para os conceitos topológicos é [57].

Definição 1.1.1. Seja X um espaço topológico. Dizemos que um ponto $x$ de $X$ tem uma base enumerável se existe uma coleção $\left\{U_{n}\right\}$ de vizinhanças (conjuntos abertos) de $x$ tal que qualquer vizinhança de $x$ contém no mínimo um dos conjuntos $U_{n}$.

Definição 1.1.2. Um espaço topológico $X$ é primeiro enumerável se cada ponto de $X$ possui uma base enumerável.

Exemplos de tais espaços são os espaços métricos.

Definição 1.1.3. Sejam $X$ e $Y$ espaços topológicos. Uma aplicação $f: X \rightarrow Y$ é sequencialmente contínua quando preserva limites sequenciais: se uma sequência $\left\{x_{n}\right\}$ converge para $x$ em $X$, então a sequência $\left\{f\left(x_{n}\right)\right\}$ converge para $f(x)$ em $Y$. 
O próximo resultado, encontrado em [57], relaciona estes conceitos.

Teorema 1.1.4. Sejam $X$ e $Y$ espaços topológicos. Se $f: X \rightarrow Y$ é uma aplicação contínua, então $f$ é sequencialmente contínua. A recíproca vale quando $X$ é primeiro enumerável.

Definição 1.1.5. Um espaço topológico $X$ é localmente compacto se para cada $x \in X$ existe um subconjunto compacto $C_{x}$ de $X$ que contém uma vizinhança de $x$.

No que diz respeito à convergência uniforme de sequências e séries de funções, utilizamos os seguintes resultados encontrados em [50, 76].

Teorema 1.1.6 (Teorema de Dini). Sejam X um espaço topológico compacto e $f_{n}: X \rightarrow$ $\mathbb{R}$ uma sequência de funções contínuas convergindo pontualmente para uma função contínua $f: X \rightarrow \mathbb{R}$. Se cada sequência $\left\{f_{n}(x)\right\}, x \in X$, é monótona crescente, então $\left\{f_{n}\right\}$ converge uniformemente para $f$ em $X$.

Teorema 1.1.7 (Critério de Cauchy). Sejam $X$ um espaço métrico e $E \subset X$. Uma sequência $\left\{f_{n}\right\}$ de funções reais definidas sobre $E$ converge uniformemente sobre $E$ se, e somente se, para cada $\varepsilon>0$ existe um inteiro $N$ tal que $m \geq N$ e $n \geq N$ implicam

$$
\left|f_{n}(x)-f_{m}(x)\right|<\varepsilon, \quad x \in E
$$

Para sequências numéricas, usamos os seguintes símbolos para tratar da ordem de convergência.

Definição 1.1.8. Sejam $\left\{a_{n}\right\}$ e $\left\{b_{n}\right\}$ duas sequências numéricas. Suponha que existe $n_{0} \in \mathbb{N}$ tal que $b_{n} \neq 0, n \geq n_{0}$. Denotamos $a_{n}=o\left(b_{n}\right)$ no caso da sequência $\left\{a_{n} b_{n}^{-1}\right\}$ convergir para 0 . Se $\left\{a_{n} b_{n}^{-1}, n=n_{0}, n_{0}+1, \ldots\right\}$ é limitada, escrevemos $a_{n}=O\left(b_{n}\right)$.

Os próximos resultados seguem diretamente da definição anterior. Demonstrações podem ser encontradas em [13].

Proposição 1.1.9. Nas condições da Definição 1.1.8, com $n \geq n_{0}$, existe uma constante $c>0$ tal que $\left|a_{n}\right| \leq c\left|b_{n}\right|$ se, e somente se, $a_{n}=O\left(b_{n}\right)$.

Corolário 1.1.10. Sejam $a, b \in(0, \infty)$ e $\left\{a_{n}\right\}$ uma sequência de termos reais positivos tal que $\lim _{n \rightarrow \infty} a_{n}=\infty$. Então, $a a_{n}+b=O\left(a_{n}\right)$. Além disso, existe uma constante positiva c tal que $a a_{n}+b \leq c a_{n}, k \in \mathbb{N}$.

No que segue, consideramos uma série numérica $\sum_{n} a_{n}$. A convergência desta série implica na convergência de $\left\{a_{n}\right\}$ para 0 . Menos conhecido é o fato de que se $\left\{a_{n}\right\}$ é decrescente, contém somente termos positivos e $\sum_{n} a_{n}$ converge, então a sequência 
$\left\{n a_{n}\right\}$ converge para 0 (veja Teorema 1.1 .9 em [27]). O próximo teorema, encontrado em [34, 44], generaliza este resultado e mostra como obter a ordem de convergência de uma sequência através da convergência de séries.

Teorema 1.1.11. Sejam $\left\{a_{n}\right\}$ uma sequência decrescente de números reais positivos e $\alpha$ e $\beta$ constantes positivas. Se $\sum_{n=1}^{\infty} n^{\alpha} a_{n}^{\beta}$ converge, então $a_{n}=o\left(n^{-(\alpha+1) / \beta}\right)$.

Propriedades aritméticas das séries serão muito utilizadas nas demonstrações de nossos resultados sobre decaimento de autovalores. Por este motivo, relembramos algumas definições e resultados retirados de [49]. Começamos com a associatividade: dada uma série convergente, a inserção de parênteses entre seus termos não altera o seu limite. O mesmo não ocorre com a dissociatividade, pois a série obtida ao dissociarmos os termos de uma série convergente pode não convergir. Por exemplo, a série $0+0+\ldots$ é convergente. Por outro lado, escrevendo $0=1-1$ obtemos, por dissociação, a série $1-1+1-1+\ldots$, que é divergente. Entretanto, a dissociatividade é possível no caso da série em questão ser absolutamente convergente e seus termos serem decompostos em somas finitas com parcelas com o mesmo sinal, pois neste caso o limite não é alterado. A soma de séries convergentes também é uma operação válida, isto é, se $\sum_{n} a_{n}=t$ e $\sum_{n} b_{n}=s$, então $\sum_{n}\left(a_{n}+b_{n}\right)=t+s$. Finalmente, dizemos que uma série $\sum_{n} a_{n}$ é comutativamente convergente quando, para toda bijeção $\varphi: \mathbb{N} \rightarrow \mathbb{N}$, a série $\sum_{n} a_{\varphi(n)}$ é convergente e $\sum_{n} a_{n}=\sum_{n} a_{\varphi(n)}$. Toda série absolutamente convergente é comutativamente convergente.

\subsection{Teoria da medida}

Nesta seção recordamos um pouco da terminologia básica sobre teoria da medida e resultados importantes que são utilizados em demonstrações presentes no texto. Estas e outras informações sobre o tema podem ser encontradas em [25, 31].

Definição 1.2.1. Seja $(X, \mu)=(X, \mathcal{M}, \mu)$ um espaço de medida. Se $\mu(X)<\infty(e$, consequentemente, $\mu(E)<\infty$, para todo $E \in \mathcal{M})$ dizemos que a medida $\mu$ é finita. Se $X=\cup_{n \geq 1} E_{n}$, com $E_{n} \in \mathcal{M}$ e $\mu\left(E_{n}\right)<\infty$, para todo $n \in \mathbb{N}$, então dizemos que $\mu$ é $\sigma$-finita ou que $X$ é $\sigma$-finito com relação a $\mu$ (ou simplesmente $\sigma$-finito). Se $E \in \mathcal{M} e$ $\mu(E)=0$, dizemos que $E$ tem medida nula. Quando uma afirmação sobre elementos de $X$ é verdadeira em todo $X$ exceto em um conjunto de medida nula, dizemos que tal afirmação vale quase sempre (e abreviamos q.s.), ou para quase todo $x \in X$. No caso em que $X$ é um espaço topológico e $\mu$ é uma medida de Borel, dizemos que $\mu$ é localmente finita quando $\mu(K)<\infty$, para todo $K \subset X$ compacto e dizemos que $\mu$ é não degenerada se $\mu(A)>0$, para todo subconjunto aberto não-vazio $A$ de $X$. 
Agora, relembramos um pouco sobre os espaços $L^{p}$.

Definição 1.2.2. Sejam $(X, \mu)$ um espaço de medida e $p \in(0, \infty]$. Definimos

$$
L^{p}(X, \mu):=\left\{f: X \rightarrow \mathbb{C}: f \text { é } \mu \text {-mensurável e }\|f\|_{p}<\infty\right\}
$$

onde

$$
\|f\|_{p}:=\left[\int_{X}|f(x)|^{p} d \mu(x)\right]^{1 / p}, \quad 0<p<\infty
$$

$e$

$$
\|f\|_{\infty}:=e s s \sup _{x \in X}\{|f(x)|\}=\inf \{a \geq 0: \mu(\{x \in X:|f(x)|>a\})=0\} .
$$

O conjunto $L^{p}(X, \mu)$, para $p \in(0, \infty]$, torna-se um espaço vetorial quando identificamos quaisquer duas funções $f$ e $g$ de $L^{p}(X, \mu)$ que coincidem q.s. (e, neste caso, escrevemos $f=g$ q.s.). No caso em que $X$ é $\mathbb{R}^{m+1}$, ou algum subconjunto de $\mathbb{R}^{m+1}$ (a esfera, por exemplo), sempre usaremos a medida de Lebesgue usual de $\mathbb{R}^{m}$ ou, respectivamente, a medida de Lebesgue induzida. No contexto de $L^{p}(X \times Y, \mu \times \nu)$, a medida $\mu \times \nu$ é a medida produto correspondente. Propriedades importantes dos espaços $L^{p}$ estão presentes no teorema seguinte.

Teorema 1.2.3. Para $p \geq 1$, valem as seguintes propriedades:

(i) O espaço $\left(L^{p}(X, \mu),\|\cdot\|_{p}\right)$ é um espaço de Banach;

(ii) $L^{2}(X, \mu)$ é um espaço de Hilbert com produto interno dado por

$$
\langle f, g\rangle_{2}:=\int_{X} f(x) \overline{g(x)} d \mu(x), \quad f, g \in L^{2}(X, \mu) .
$$

No desenvolvimento do trabalho fazemos muitas manipulações de integrais, por este motivo terminamos a seção com alguns resultados úteis sobre o assunto.

Teorema 1.2.4 (Desigualdade de Hölder). Sejam $(X, \mu)$ um espaço de medida e $p \in$ $[1, \infty]$. Considere o expoente conjugado de $p$, ou seja, o número q que satisfaz $p^{-1}+$ $q^{-1}=1$. Se $f$ e g são funções mensuráveis em $X$, então

$$
\|f g\|_{1} \leq\|f\|_{p}\|g\|_{q}
$$

Em particular, se $f \in L^{p}(X, \mu)$ e $g \in L^{q}(X, \nu)$, então $f g \in L^{1}(X, \mu)$.

Teorema 1.2.5 (Convergência Dominada). Seja $\left\{f_{n}\right\}$ uma sequência em $L^{1}(X, \mu)$ que satisfaz:

(i) $\lim _{n \rightarrow \infty} f_{n}=f$ q.s.; 
(ii) Existe uma função $g \in L^{1}(X, \mu)$ tal que $\left|f_{n}\right| \leq g$ q.s., para todo $n$.

Então, $f \in L^{1}(X, \mu) e$

$$
\int_{X} f(x) d \mu(x)=\lim _{n \rightarrow \infty} \int_{X} f_{n}(x) d \mu(x)
$$

O próximo teorema garante a iteração de integrais em espaços produto.

Teorema 1.2.6 (Fubini). Sejam $(X, \mu)$ e $(Y, \nu)$ espaços de medida completos (ou $\sigma$ finitos) e $f \in L^{1}(X \times Y, \mu \times \nu)$. Neste caso, $f(x, \cdot) \in L^{1}(Y, \nu)$ para quase todo $x e$ $f(\cdot, y) \in L^{1}(X, \mu)$ para quase todo $y$. As funções definidas quase sempre,

$$
g(x)=\int_{Y} f(x, y) d \nu(y) \quad e \quad h(y)=\int_{X} f(x, y) d \mu(x),
$$

são elementos de $L^{1}(X, \mu)$ e $L^{1}(Y, \nu)$, respectivamente. Além disso, vale a fórmula

$$
\int_{X \times Y} f d(\mu \times \nu)=\int_{X}\left[\int_{Y} f(x, y) d \nu(y)\right] d \mu(x)=\int_{Y}\left[\int_{X} f(x, y) d \mu(x)\right] d \nu(y) .
$$

Teorema 1.2.7 (Desigualdade de Minkowski para integrais). $\operatorname{Sejam}(X, \mathcal{M}, \mu)$ e $(Y, \mathcal{N}, \nu)$ espaços com medidas $\sigma$-finitas e $f$ uma função $(\mathcal{M} \otimes \mathcal{N})$-mensurável sobre $X \times Y$. Se $1 \leq p \leq \infty, f(\cdot, y) \in L^{p}(\mu)$ q.s., e a função $y \mapsto\|f(\cdot, y)\|_{p}$ é $\nu$-integrável, então $f(x, \cdot) \in L^{1}(\nu)$ q.s., a função $x \mapsto \int f(x, y) d \nu(y)$ pertence a $L^{p}(\mu) e$

$$
\left\|\int_{Y} f(\cdot, y) d \nu(y)\right\|_{p} \leq \int_{Y}\|f(\cdot, y)\|_{p} d \nu(y) .
$$

Encerramos esta seção apresentando um ambiente que garante a existência de bases ortogonais enumeráveis para espaços de funções de quadrado integrável ([52, p. 92]).

Teorema 1.2.8. Seja $(X, \mathcal{M}, \mu)$ um espaço de medida. Suponha que a $\sigma$-álgebra $\mathcal{M}$ é enumeravelmente gerada (a menos de conjuntos com medida nula) e $X$ é $\sigma$-finito. Então, $L^{2}(X, \mu)$ é separável.

\subsection{Análise funcional}

Esta seção contém os pré-requisitos de análise funcional utilizados ao longo do trabalho. Detalhes e resultados adicionais podem ser encontrados nas referências [31, 35, 61, 84.

Denotamos por $\mathcal{X}$ um espaço vetorial normado, munido da norma $\|\cdot\|_{\mathcal{X}}$. Além disso, escrevemos $\|x\|_{\mathcal{X}}^{2}=\langle x, x\rangle_{\mathcal{X}}, x \in \mathcal{X}$, sempre que $\mathcal{X}$ estiver munido de um produto interno $\langle\cdot, \cdot\rangle_{\mathcal{X}}$. Tratamos apenas dos espaços vetoriais de dimensão infinita sobre $\mathbb{R}$ ou $\mathbb{C}$. Começamos relembrando a clássica desigualdade de Cauchy-Schwarz. 
Teorema 1.3.1 (Desigualdade de Cauchy-Schwarz). Se $\mathcal{X}$ é um espaço com produto interno $\langle\cdot, \cdot\rangle_{\mathcal{X}}$, então

$$
\left|\langle x, y\rangle_{\mathcal{X}}\right| \leq\|x\|_{\mathcal{X}}\|y\|_{\mathcal{X}}, \quad x, y \in \mathcal{X}
$$

Os próximos resultados estão relacionados com propriedades de conjuntos ortonormais de espaços de Hilbert.

Teorema 1.3.2 (Desigualdade de Bessel). Se $\mathcal{H}$ é um espaço de Hilbert e $\left\{x_{\alpha}\right\}_{\alpha \in A}$ é um subconjunto ortonormal de $\mathcal{H}$, então

$$
\sum_{\alpha \in A}\left|\left\langle y, x_{\alpha}\right\rangle_{\mathcal{H}}\right|^{2} \leq\|y\|_{\mathcal{H}}^{2}, \quad y \in \mathcal{H}
$$

Lembramos que o somatório acima representa de fato a soma de uma série, ou seja, os elementos desta soma podem ser não nulos apenas em um conjunto enumerável de índices. O mesmo comentário se aplica a outros somatórios do texto. Este resultado pode ser melhorado quando o conjunto em questão é uma base ortonormal do espaço.

Teorema 1.3.3 (Identidade de Parseval). Se $\mathcal{H}$ é um espaço de Hilbert $e\left\{x_{\alpha}\right\}_{\alpha \in A}$ é uma base ortonormal de $\mathcal{H}$, então

$$
y=\sum_{\alpha \in A}\left\langle y, x_{\alpha}\right\rangle_{\mathcal{H}} x_{\alpha}, \quad y \in \mathcal{H}
$$

$e$

$$
\|y\|_{\mathcal{H}}^{2}=\sum_{\alpha \in A}\left|\left\langle y, x_{\alpha}\right\rangle_{\mathcal{H}}\right|^{2}, \quad y \in \mathcal{H}
$$

Além disso, se $\left\{c_{n}\right\}$ é uma sequência de números complexos tal que $\sum_{n=1}^{\infty}\left|c_{n}\right|^{2}<\infty$, então $x:=\sum_{n=1}^{\infty} c_{n} x_{\alpha_{n}}$ é um elemento de $\mathcal{H}$ e $c_{n}=\left\langle x, x_{\alpha_{n}}\right\rangle_{\mathcal{H}}$.

O próximo resultado é um caso particular da segunda parte do resultado anterior encontrado em [76, p. 330].

Teorema 1.3.4 (Teorema de Riesz-Fischer). Sejam $\left\{\phi_{n}\right\}$ uma sequência ortonormal em $L^{2}(X)$ e $\left\{c_{n}\right\}$ uma sequência de números complexos tais que $\sum_{n}\left|c_{n}\right|^{2}<\infty$. Defina $s_{n}=c_{1} \phi_{1}+\cdots+c_{n} \phi_{n}$. Então, existe $f \in L^{2}(X, \mu)$ tal que $\left\{s_{n}\right\}$ converge para $f$ em $L^{2}(X)$.

Seguimos recordando alguns resultados sobre transformações lineares. Sejam $\mathcal{X}$ e $\mathcal{Y}$ espaços vetoriais normados. O espaço $\mathcal{L}(\mathcal{X}, \mathcal{Y})$ de todas as transformações lineares de $\mathcal{X}$ em $\mathcal{Y}$ é ele mesmo um espaço vetorial. Denotamos por $\mathcal{B}(\mathcal{X}, \mathcal{Y})$ o subespaço formado 
pelas transformações lineares limitadas (ou contínuas). Podemos munir $\mathcal{B}(\mathcal{X}, \mathcal{Y})$ com a norma

$$
\|T\|_{\mathcal{B}(\mathcal{X}, \mathcal{Y})}:=\sup \left\{\|T(x)\|_{\mathcal{Y}}: x \in \mathcal{X},\|x\|_{\mathcal{X}}=1\right\}
$$

Quando $\mathcal{X}=\mathcal{Y}$, escrevemos $\mathcal{L}(\mathcal{X}, \mathcal{Y})=\mathcal{L}(\mathcal{X})$ e $\mathcal{B}(\mathcal{X}, \mathcal{Y})=\mathcal{B}(\mathcal{X})$ e chamamos seus elementos de operadores lineares.

Teorema 1.3.5. Sejam $\mathcal{X}$ um espaço vetorial normado e $\mathcal{Y}$ um espaço de Banach. Então, $\mathcal{B}(\mathcal{X}, \mathcal{Y})$ é um espaço de Banach.

Por simplicidade, denotamos $\|T\|:=\|T\|_{\mathcal{B}(\mathcal{X}, \mathcal{Y})}$. No caso em que $\mathcal{X}$ é um espaço de Hilbert e $\mathcal{Y}$ é $\mathbb{R}$ ou $\mathbb{C}$, o teorema anterior pode ser melhorado pelo Teorema da Representação de Riesz que garante a existência de um elemento $y=y(T) \in \mathcal{X}$ tal que

$$
T(x)=\langle x, y\rangle_{\mathcal{X}}, \quad x \in \mathcal{X},
$$

para cada $T \in \mathcal{B}(\mathcal{X}, \mathcal{Y})$. Logo, $\mathcal{B}(\mathcal{X}, \mathcal{Y})$ é isomorfo a $\mathcal{X}$ quando $\mathcal{Y}$ é igual a $\mathbb{R}$ ou $\mathbb{C}$.

Uma importante classe de transformações se origina do seguinte teorema.

Teorema 1.3.6. Sejam $\mathcal{H}_{1}$ e $\mathcal{H}_{2}$ espaços de Hilbert. Se $T \in \mathcal{B}\left(\mathcal{H}_{1}, \mathcal{H}_{2}\right)$, então existe uma única transformação linear $T^{*} \in \mathcal{B}\left(\mathcal{H}_{2}, \mathcal{H}_{1}\right)$ tal que

$$
\langle T(x), y\rangle_{\mathcal{H}_{2}}=\left\langle x, T^{*}(y)\right\rangle_{\mathcal{H}_{1}}, \quad x \in \mathcal{H}_{1}, \quad y \in \mathcal{H}_{2}
$$

A transformação $T^{*}$ descrita no teorema anterior é denominada transformação adjunta de $T$. Um operador $T$ é autoadjunto quando $T=T^{*}$.

Outra classe de operadores indispensável aos nossos estudos é apresentada a seguir.

Definição 1.3.7. Seja $\mathcal{X}$ um espaço de Banach. Um operador $T \in \mathcal{B}(\mathcal{X})$ é compacto se a imagem de cada sequência limitada de $\mathcal{X}$ possui uma subsequência convergente. Denotamos o espaço dos operadores compactos por $\mathcal{B}_{0}(\mathcal{X})$.

Exemplos elementares de operadores compactos são os operadores de posto finito. O resultado seguinte mostra, além de outras coisas, que todo operador compacto pode ser aproximado por operadores de posto finito.

Teorema 1.3.8. Sejam $\mathcal{H}$ um espaço de Hilbert e $T \in \mathcal{B}(\mathcal{H})$. Então, $T$ é compacto se, e somente se, $T^{*}$ é compacto. Além disso, o conjunto dos operadores de posto finito é denso no espaço (de Banach) dos operadores compactos.

Outra maneira de obtermos operadores compactos é sugerida pelo teorema abaixo. 
Teorema 1.3.9. Sejam $\mathcal{X}$ um espaço de Banach e $T$ e $S$ operadores limitados. Se $T$ ou $S$ é compacto, então as composições $S T$ e TS são operadores compactos.

Vejamos agora a principal classe de operadores lineares a ser usada neste trabalho.

Definição 1.3.10. Seja $\mathcal{H}$ um espaço de Hilbert. Um operador $T \in \mathcal{B}(\mathcal{H})$ é positivo quando

$$
\langle T(x), x\rangle_{\mathcal{H}} \geq 0, \quad x \in \mathcal{H}
$$

Se $T \in \mathcal{B}(\mathcal{H})$ é positivo, escrevemos $T \geq 0$. Se $T_{1}, T_{2} \in \mathcal{B}(\mathcal{H})$, escrevemos $T_{1} \geq T_{2}$ para indicar que $T_{1}-T_{2} \geq 0$. Se $T \in \mathcal{B}(\mathcal{H})$, então $T^{*} T \geq 0$, uma vez que

$$
\left\langle T^{*} T(x), x\right\rangle_{\mathcal{H}}=\langle T(x), T(x)\rangle_{\mathcal{H}}=\|T(x)\|_{\mathcal{H}}^{2} \geq 0, \quad x \in \mathcal{H}
$$

Além disso, é fácil ver quer $T^{*} T$ é autoadjunto. O próximo resultado apresenta um contexto onde este fato pode ser visto de forma mais geral.

Teorema 1.3.11. Sejam $\mathcal{H}$ um espaço de Hilbert complexo e $T \in \mathcal{B}(\mathcal{H})$. Se $T$ é positivo, então $T$ é autoadjunto.

Outra importante característica dos operadores positivos é o fato de possuirem uma única raíz quadrada positiva ([79, p.231]). Com maior generalidade, temos os seguinte teorema.

Teorema 1.3.12 (Lema da Raíz $n$-ésima). Sejam $\mathcal{H}$ um espaço de Hilbert e $T \in \mathcal{B}(\mathcal{H})$. Se $T$ é positivo e n é um inteiro maior que 0, então existe um único operador positivo $\mathcal{S}$ em $\mathcal{B}(\mathcal{H})$ tal que $\mathcal{S}^{n}=T$.

O operador $\mathcal{S}$ descrito acima é usualmente denotado por $\sqrt[n]{T}$ ou $T^{1 / n}$ e chamado de raíz $n$-ésima de $T$. Se $T \in \mathcal{B}(\mathcal{H})$, definimos $|T|:=\sqrt{T^{*} T}$. Observe que $|T|=T$ quando este é autoadjunto e positivo.

Teorema 1.3.13. Sejam $\mathcal{H}$ um espaço de Hilbert e $T \in \mathcal{B}(\mathcal{H})$. Então, $T$ é compacto se, e somente se, $|T|$ é compacto.

Estamos prontos para apresentar as principais ferramentas que utilizamos no estudo do comportamento assintótico de autovalores de operadores compactos e positivos. A maioria destes resultados pertence a um ramo da Análise Funcional conhecido como teoria espectral de operadores compactos. As referências básicas onde tais resultados podem ser encontrados são [35, 61, 79, 84]. No caso de operadores compactos sobre espaços de Hilbert, o resultado mais básico é o teorema seguinte. 
Teorema 1.3.14 (Hilbert-Schmidt). Seja $T \in \mathcal{B}_{0}(\mathcal{H})$. Se $T$ é autoadjunto, então existem um subconjunto ortonormal $\left\{x_{n}\right\}$ de $\mathcal{H}$ e $\left\{\lambda_{n}(T)\right\} \subset \mathbb{R}$ tais que

$$
T(x)=\sum_{n=1}^{\infty} \lambda_{n}(T)\left\langle x, x_{n}\right\rangle_{\mathcal{H}} x_{n}, \quad x \in \mathcal{H},
$$

$\operatorname{com}\left|\lambda_{1}(T)\right| \geq\left|\lambda_{2}(T)\right| \geq \cdots \geq 0$ e $\lim _{n \rightarrow \infty} \lambda_{n}(T)=0$.

No teorema anterior, o símbolo $\lambda_{n}(T)$ representa os autovalores do operador $T$ e a sequência $\left\{\lambda_{n}(T)\right\}$ leva em consideração possíveis repetições geradas pela multiplicidade algébrica de tais autovalores.

Corolário 1.3.15. Nas condições do Teorema de Hilbert-Schmidt:

(i) Se $T \geq 0$, então $\lambda_{n}(T) \geq 0, n=1,2, \ldots$;

(ii) Se $\mathcal{H}$ é separável, então podemos supor que $\left\{x_{n}\right\}$ é uma base ortonormal do espaço.

Demonstração: Para provar $(i)$ basta notar que se $T(x)=\lambda(T) x$ e $x \neq 0$, então $0 \leq\langle T(x), x\rangle_{\mathcal{H}}=\lambda(T)\langle x, x\rangle_{\mathcal{H}}$. O item $(i i)$ segue da equivalência entre a separabilidade de $\mathcal{H}$ e a existência de base ortonormal enumerável.

Se $T$ é um operador compacto sobre um espaço de Hilbert, já sabemos que $|T|$ é autoadjunto, compacto e positivo. Logo, o Teorema de Hilbert-Schmidt é aplicável para este operador.

Definição 1.3.16. Seja $T \in \mathcal{B}_{0}(\mathcal{H})$. Os valores singulares $s_{n}(T)$ de $T$ são os autovalores de $|T|$, isto é, $s_{n}(T)=\lambda_{n}(|T|)$.

Por mais claro e repetitivo que isto possa parecer, gostaríamos de ressaltar que a disposição dos valores singulares de $T \in \mathcal{B}_{0}(\mathcal{H})$ segue a ordenação imposta pelo Teorema de Hilbert-Schmidt, em ordem decrescente e levando em consideração a multiplicidade algébrica dos mesmos quando vistos como autovalores de $|T|$.

O próximo resultado descreve algumas propriedades dos valores singulares que utilizamos em nossas demonstrações. Elas podem ser encontradas provadas em referências sobre teoria de operadores, como por exemplo [34, 35, 43, 67], e dependem da ordenação dos valores singulares na forma descrita anteriormente.

Teorema 1.3.17. Seja $T \in \mathcal{B}_{0}(\mathcal{H})$. As seguintes afirmações são verdadeiras.

(i) Se $A \in \mathcal{B}(\mathcal{H})$, então

$$
\max \left\{s_{n}(A T), s_{n}(T A)\right\} \leq\|A\| s_{n}(T), \quad n=1,2, \ldots ;
$$

(ii) Se $A \in \mathcal{L}(\mathcal{H})$ possui posto no máximo l, então

$$
s_{n+l}(T) \leq s_{n}(T+A), \quad n=1,2, \ldots ;
$$


(iii) Se $A \in \mathcal{B}_{0}(\mathcal{H})$, então

$$
s_{n+k-1}(A T) \leq s_{n}(A) s_{k}(T), \quad n, k=1,2, \ldots .
$$

O próximo resultado mostra um contexto onde podemos relacionar os valores singulares e os autovalores de operadores compactos.

Teorema 1.3.18. Se $T \in \mathcal{B}_{0}(\mathcal{H})$ é autoadjunto, então

$$
s_{n}(T)=\left|\lambda_{n}(T)\right|, \quad n=1,2, \ldots
$$

De acordo com os resultados apresentados anteriormente, se $T$ é um operador compacto, autoadjunto e positivo sobre um espaço de Hilbert, então $s_{n}(T)=\lambda_{n}(T)$, $n=1,2, \ldots$

Outra classe de operadores utilizada em nossos resultados é a classe formada pelos operadores nucleares que descrevemos a seguir.

Definição 1.3.19. Sejam $\mathcal{H}$ um espaço de Hilbert e $\left\{x_{\alpha}\right\}_{\alpha \in A}$ uma base ortonormal de H. Se $T \in \mathcal{B}(\mathcal{H})$, o traço de $T$ é definido por

$$
\operatorname{tr}(T):=\sum_{\alpha \in A}\left\langle T^{*} T\left(x_{\alpha}\right), x_{\alpha}\right\rangle_{\mathcal{H}}^{1 / 2}
$$

Pode-se provar que o traço de um operador independe da escolha da base de $\mathcal{H}$. Mais ainda, se $\mathcal{H}$ é separável e $T$ é compacto e positivo, então o traço de $T$ se reduz a

$$
\operatorname{tr}(T):=\sum_{n=1}^{\infty} s_{n}(T)
$$

Seguimos a seção introduzindo mais uma categoria de operadores. Começamos com uma definição.

Definição 1.3.20. Seja $\mathcal{H}$ espaço de Hilbert. Um operador $T \in \mathcal{B}_{0}(\mathcal{H})$ é nuclear quando $\operatorname{tr}(|T|)<\infty$. O espaço dos operadores nucleares é denotado por $\mathcal{B}_{1}(\mathcal{H})$.

É possível provar que $\mathcal{B}_{1}(\mathcal{H})$ é um subespaço vetorial de $\mathcal{B}(\mathcal{H})$.

No restante da seção abandonamos o cenário geral de espaços de Hilbert e apresentamos o tipo de operador a ser analisado no decorrer da tese.

Definição 1.3.21. Considere um operador linear $T: L^{2}(X, \mu) \rightarrow L^{2}(X, \mu)$. Se existir uma aplicação $K: X \times X \rightarrow \mathbb{C}$ para a qual

$$
T(f)(x)=\int_{X} K(x, y) f(y) d \mu(y), \quad f \in L^{2}(X, \mu), \quad x \in X \text { q.s. }
$$

dizemos que $T$ é um operador integral sobre $L^{2}(X, \mu)$. Neste caso, escrevemos $T=\mathcal{K}$ e dizemos que $K$ é o núcleo gerador deste operador. 
Quando o núcleo $K$ pertence a $L^{2}(X \times X, \mu \times \mu)$, onde $\mu$ é $\sigma$-finita, o Teorema de Fubini e a desigualdade de Cauchy-Schwarz garantem que

$$
\begin{aligned}
\|\mathcal{K}(f)\|_{2}^{2} & =\int_{X}|\mathcal{K}(f)(x)|^{2} d \mu(x) \\
& =\int_{X}\left|\int_{X} K(x, y) f(y) d \mu(y)\right|^{2} d \mu(x) \\
& \leq \int_{X}\left(\left(\int_{X}|K(x, y)|^{2} d \mu(y)\right)^{1 / 2}\left(\int_{X}|f(y)|^{2} d \mu(y)\right)^{1 / 2}\right)^{2} d \mu(x) \\
& =\|K\|_{2}^{2}\|f\|_{2}^{2}, \quad f \in L^{2}(X, \mu),
\end{aligned}
$$

ou seja, $\|\mathcal{K}\| \leq\|K\|_{2}$, o que significa que $\mathcal{K}$ é limitado. Outra propriedade destes operadores é dada a seguir.

Teorema 1.3.22. Seja $K$ um núcleo em $L^{2}(X \times X, \mu \times \mu)$. Então, $\mathcal{K}$ é compacto.

O seguinte resultado adicional sobre valores singulares de operadores integrais está provado em [43].

Teorema 1.3.23. Sejam $(X, \mu)$ um espaço de medida finita e $K \in L^{2}(X \times X)$, então

$$
\sum_{n=1}^{\infty} s_{n}^{2}(\mathcal{K})=\|K\|_{2}^{2}
$$

Lembramos que um núcleo $K$ é hermitiano quando $K(x, y)=\overline{K(y, x)}, x, y \in X$. Dito isto, temos o seguinte resultado que motiva o estudo realizado no Capítulo 2 .

Teorema 1.3.24. Seja $K$ um núcleo hermitiano em $L^{2}(X \times X, \mu \times \mu)$. Suponha que toda base ortonormal $\left\{\phi_{\alpha}\right\}_{\alpha \in A}$ de $L^{2}(X, \mu)$ é tal $\left\{\phi_{\alpha} \otimes \phi_{\beta}\right\}_{\alpha, \beta \in A}$ é base ortonormal de $L^{2}(X \times X, \mu \times \mu)$. Então, existe uma sequência $\left\{\lambda_{n}(\mathcal{K})\right\} \subset \mathbb{R}$ e um conjunto ortonormal $\left\{\phi_{n}\right\}$ de $L^{2}(X, \mu)$ tais que

$$
K=\sum_{n=1}^{\infty} \lambda_{n}(\mathcal{K}) \phi_{n} \otimes \overline{\phi_{n}}
$$

com convergência em $L^{2}(X \times X, \mu \times \mu)$.

Demonstração: Como $K$ é hermitiano, então $\mathcal{K}$ é autoadjunto. O Lema 1.3 .22 revela que $\mathcal{K}$ é compacto. O Teorema 1.3 .14 garante que existem um conjunto ortonormal $\left\{\phi_{n}\right\}$ de $L^{2}(X, \mu)$ e uma sequência $\left\{\lambda_{n}(\mathcal{K})\right\} \subset \mathbb{R}$, convergente para 0 , tais que $\mathcal{K}\left(\phi_{n}\right)=$ $\lambda_{n}(\mathcal{K}) \phi_{n} \mathrm{e}$

$$
\mathcal{K}(f)=\sum_{n=1}^{\infty} \lambda_{n}(\mathcal{K})\left\langle f, \phi_{n}\right\rangle_{2} \phi_{n}, \quad f \in L^{2}(X, \mu)
$$


Usando o Lema de Zorn podemos completar, se necessário, o conjunto $\left\{\phi_{n}\right\}$ para obter uma base ortonormal $\left\{\phi_{\alpha}\right\}_{\alpha \in A}$ de $L^{2}(X, \mu)$. Como o conjunto $\left\{\phi_{\alpha} \otimes \overline{\phi_{\beta}}\right\}_{\alpha, \beta \in A}$ é uma base ortonormal de $L^{2}(X \times X, \mu \times \mu)$, a identidade de Parseval garante que

$$
K=\sum_{\alpha, \beta \in A}\left\langle K, \phi_{\alpha} \otimes \overline{\phi_{\beta}}\right\rangle_{2} \phi_{\alpha} \otimes \overline{\phi_{\beta}}=\sum_{n=1}^{\infty} \lambda_{n}(\mathcal{K}) \phi_{n} \otimes \overline{\phi_{n}}
$$

uma vez que, pelo Teorema de Fubini,

$$
\delta_{\alpha, \beta} \lambda_{\alpha}(\mathcal{K})=\left\langle\mathcal{K}\left(\phi_{\alpha}\right), \phi_{\beta}\right\rangle_{2}=\left\langle\mathcal{K}, \phi_{\alpha} \otimes \overline{\phi_{\beta}}\right\rangle_{2}, \quad \alpha, \beta \in A,
$$

onde

$$
\delta_{\alpha, \beta}= \begin{cases}1 & , \alpha=\beta \\ 0 & , \alpha \neq \beta\end{cases}
$$

e $\lambda_{\alpha}(\mathcal{K})=0$ para $\phi_{\alpha}$ ortonormal a $\left\{\phi_{n}\right\}$.

Definição 1.3.25. Dizemos que um núcleo $K \in L^{2}(X \times X, \mu \times \mu)$ é $L^{2}(X, \mu)$-positivo definido se

$$
\int_{X} \int_{X} K(x, y) f(x) \overline{f(y)} d \mu(x) d \mu(y) \geq 0, \quad f \in L^{2}(X, \mu) .
$$

Em outras palavras, $K$ é $L^{2}(X, \mu)$-positivo definido quando o operador integral gerado por $K$ é positivo. Neste caso, segue do Teorema 1.3.11 que $\mathcal{K}$ é autoadjunto.

Definição 1.3.26. Um núcleo $K: X \times X \rightarrow \mathbb{C}$ é positivo definido ([3, 55]) quando

$$
\sum_{i, j=1}^{n} \overline{c_{i}} c_{j} K\left(x_{i}, x_{j}\right) \geq 0
$$

para quaisquer $n \geq 1, x_{1}, x_{2}, \ldots, x_{n} \in X$ e $c_{1}, c_{2}, \ldots, c_{n} \in \mathbb{C}$.

Os próximos resultados, encontrados em [27, p.23], mostram as relações entre estes dois conceitos.

Teorema 1.3.27. Seja $X$ um espaço de Hausdorff localmente compacto e munido de uma medida de Radon $\mu$. Se $K: X \times X \rightarrow \mathbb{C}$ é positivo definido, contínuo e gera um operador integral limitado em $L^{2}(X, \mu)$, então ele é $L^{2}(X, \mu)$-positivo definido.

Teorema 1.3.28. Seja $X$ um espaço topológico munido de uma medida estritamente positiva $\mu$. Se $K: X \times X \rightarrow \mathbb{C}$ é $L^{2}(X, \mu)$-positivo definido e contínuo, então ele é positivo definido. 


\subsection{Os harmônicos esféricos}

Nesta seção, apresentamos alguns conceitos e resultados pertinentes à análise em $S^{m}, m \geq 2$. Mais precisamente, recordamos um pouco da teoria clássica de harmônicos esféricos. Uma ampla discussão sobre este tópico, incluindo provas e aplicações, pode ser encontrada nas referências [1, 36, 56, 78.

Denotamos por $x=\left(x_{1}, x_{2}, \ldots, x_{m+1}\right)$ um ponto genérico do espaço euclidiano $\mathbb{R}^{m+1}$, por $\|x\|=\sqrt{x_{1}^{2}+x_{2}^{2}+\cdots+x_{m+1}^{2}}$ a norma euclidiana de $x$ e por

$$
x \cdot y=x_{1} y_{1}+x_{2} y_{2}+\cdots+x_{m+1} y_{m+1}
$$

o produto interno usual em $\mathbb{R}^{m+1}$. Como mencionado anteriormente, o conjunto $S^{m}=$ $\left\{x \in \mathbb{R}^{m+1}:\|x\|=1\right\}$ é a esfera unitária $m$-dimensional centrada na origem de $\mathbb{R}^{m+1}$.

Seja $d \sigma_{m}$ o elemento de medida não normalizado sobre $S^{m}$ induzido pela medida de Lebesgue. Isto significa que

$$
\int_{S^{m}} d \sigma_{m}=\sigma_{m}
$$

onde $\sigma_{m}$ denota o valor da medida de $S^{m}$. Por exemplo, $\sigma_{2}=4 \pi$ e, no caso geral, $\sigma_{m}=2 \pi^{(m+1) / 2} / \Gamma((m+1) / 2)$, onde $\Gamma$ é a função gama usual.

Dado um multi-índice $\alpha=\left(\alpha_{1}, \ldots, \alpha_{m+1}\right) \in \mathbb{N}^{m+1}:=\{0,1, \ldots\}^{m+1}$, definimos o operador diferencial $D^{\alpha}$ por

$$
D^{\alpha}:=\frac{\partial^{|\alpha|}}{\partial x_{1}^{\alpha_{1}} \cdots \partial x_{m+1}^{\alpha_{m+1}}}=\frac{\partial^{\alpha_{1}}}{\partial x_{1}^{\alpha_{1}}} \cdots \frac{\partial^{\alpha_{m+1}}}{\partial x_{m+1}^{\alpha_{m+1}}}, \quad|\alpha|:=\alpha_{1}+\cdots+\alpha_{m+1} .
$$

O operador diferencial

$$
\Delta=\frac{\partial^{2}}{\partial x_{1}^{2}}+\frac{\partial^{2}}{\partial x_{2}^{2}}+\cdots+\frac{\partial^{2}}{\partial x_{m+1}^{2}}
$$

é chamado de laplaciano sobre $\mathbb{R}^{m+1}$. Uma aplicação $f$ definida sobre um subconjunto aberto de $\mathbb{R}^{m+1}$ é harmônica se satisfaz a equação diferencial $\Delta f=0$. Dizemos que uma função $f$ é $k$-homogênea (ou homogênea de grau $k$ ) se $f(t x)=t^{k} f(x), t>0$. Escrevemos $\mathcal{P}_{k}\left(\mathbb{R}^{m+1}\right)$ para denotar o espaço dos polinômios $k$-homogêneos de $m+1$ variáveis e $\mathcal{P}_{k}^{\Delta}\left(\mathbb{R}^{m+1}\right)=\left\{f \in \mathcal{P}_{k}\left(\mathbb{R}^{m+1}\right): \Delta f=0\right\}$ para o espaço dos polinômios harmônicos $k$-homogêneos, onde os coeficientes dos polinômios são complexos e os polinômios são vistos como funções sobre $\mathbb{R}^{m+1}$. A restrição de um elemento de $\mathcal{P}_{k}^{\Delta}\left(\mathbb{R}^{m+1}\right)$ para $S^{m}$ é chamada de $k$-harmônico esférico de dimensão $m+1$. O conjunto destes elementos é denotado por $\mathcal{H}_{k}^{m+1}$.

Escrevemos $N(m, k):=\operatorname{dim} \mathcal{H}_{k}^{m+1}$ e observamos que tais valores são dados por $N(m, 0)=1, N(m, 1)=m+1 \mathrm{e}$

$$
N(m, k)=\left(\begin{array}{c}
m+k \\
m
\end{array}\right)-\left(\begin{array}{c}
m+k-2 \\
m
\end{array}\right), \quad k \geq 2 .
$$


Uma informação muito importante a respeito destes números (e que tem uma forte influência em nossos resultados) é o fato de que

$$
\lim _{k \rightarrow \infty} \frac{N(m, k)}{k^{m-1}}=\frac{2}{(m-1) !},
$$

o que, de acordo com a Definição 1.1 .8 , significa que $N(m, k)=O\left(k^{m-1}\right)$. A identidade 1.4.6) pode ser encontrada junto com uma demonstração em [13]. Outra propriedade útil para nosso trabalho é a seguinte ([56, p.18]):

$$
N(m+1, k)=\sum_{n=0}^{k} N(m, n) .
$$

Além disso, como o volume da esfera é finito, os espaços de harmônicos esféricos podem ser vistos como subespaços de dimensão finita de $L^{2}\left(S^{m}\right)$, o espaço de todas as funções complexas definidas sobre $S^{m}$ que possuem quadrado integrável. Lembramos que neste espaço, funções que coincidem quase sempre (q.s.) são identificadas. Seguindo o Teorema 1.2.3, munimos este espaço com o produto interno usual (com a medida normalizada) dado por

$$
\langle f, g\rangle_{2}:=\frac{1}{\sigma_{m}} \int_{S^{m}} f(x) \overline{g(x)} d \sigma_{m}(x), \quad f, g \in L^{2}\left(S^{m}\right) .
$$

e com a norma associada $\|\cdot\|_{2}$, obtendo um espaço de Hilbert.

Os subespaços de harmônicos esféricos são ortogonais com respeito ao produto interno definido em 1.4.8, o que em linguagem simbólica significa que $\mathcal{H}_{k}^{m+1} \perp \mathcal{H}_{n}^{m+1}$, $n \neq k$. É comum denotar uma base ortonormal de $\mathcal{H}_{n}^{m+1}$ por $\left\{Y_{n, 1}, Y_{n, 2}, \ldots, Y_{n, N(m, n)}\right\}$. Logo, temos $\left\langle Y_{k, i}, Y_{n, j}\right\rangle=\delta_{k n}$, para $i=1, \ldots, N(m, k)$ e $j=1 \ldots, N(m, n)$, onde $\delta_{k n}$ é o delta de Kronecker. Além disso, sabemos que o conjunto das funções complexas contínuas sobre $S^{m}$ é denso em $L^{2}\left(S^{m}\right)$. Juntando estas informações, podemos concluir que $\overline{\left[\cup_{n \in \mathbb{N}} \mathcal{H}_{n}^{m+1}\right]}=L^{2}\left(S^{m}\right)$, o que significa que os harmônicos esféricos formam um subconjunto fundamental de $L^{2}\left(S^{m}\right)$. Segue que qualquer elemento $f \in L^{2}\left(S^{m}\right)$ determina de forma única elementos $f_{n} \in \mathcal{H}_{n}^{m+1}, n=0,1, \ldots$, tal que

$$
f=\sum_{n=0}^{\infty} f_{n}
$$

na topologia de $L^{2}\left(S^{m}\right)$. Tal representação é chamada de expansão condensada de $f$ em harmônicos esféricos.

A projeção ortogonal de $L^{2}\left(S^{m}\right)$ sobre $\mathcal{H}_{n}^{m+1}$ é dada pela fórmula ([56, p.35]),

$$
\Pi_{n}(f)=f_{n}=\sum_{k=1}^{N(m, n)} \hat{f}(n, k) Y_{n, k}
$$


Os coeficientes de Fourier-Legendre aparecendo na equação anterior podem ser calculados pela fórmula

$$
\hat{f}(n, k)=\frac{1}{\sigma_{m}} \int_{S^{m}} f \overline{Y_{n, k}} d \sigma_{m}
$$

O resultado seguinte pode ser provado usando a ortogonalidade dos harmônicos esféricos ([56, p. 27]).

Teorema 1.4.1 (Teorema da Adição). Sejam $\left\{Y_{n, 1}, Y_{n, 2}, \ldots, Y_{n, N(m . n)}\right\}$ uma base ortonormal de $\mathcal{H}_{n}^{m+1}$ e $P_{n}^{m}$ o polinômio de Legendre de grau $n$ e dimensão $m+1$. Então,

$$
N(m, n) P_{n}^{m}(x \cdot y)=\sum_{j=1}^{N(m, n)} Y_{n, j}(x) \overline{Y_{n, j}(y)} .
$$

Segue quase imediatamente do teorema que

$$
\left|P_{n}^{m}(t)\right| \leq 1, \quad-1 \leq t \leq 1
$$

\subsection{0 operador de Laplace-Beltrami}

Nosso objetivo nesta seção é relembrar a noção de diferenciabilidade usual de aplicações definidas na esfera, o operador diferencial de Laplace-Beltrami e sua relação com os harmônicos esféricos. Informações mais detalhadas sobre este assunto podem ser encontradas em [1, 13, 36, 56, 78]. A origem de $\mathbb{R}^{m+1}$ será denotada por 0 , enquanto que $\mathbb{R}_{*}^{m+1}:=\left\{x \in \mathbb{R}^{m+1}: x \neq 0\right\}$.

Em geral, uma hipersuperfície $S \subset \mathbb{R}^{m+1}$ possui um operador laplaciano associado o qual é derivado do laplaciano $\Delta$ da seguinte forma: dada uma função qualquer $f$ definida sobre $S$, considere sua extensão $F$ que é constante ao longo de retas que são normais a $S$. O laplaciano de $f$ é obtido pela restrição de $\Delta F$ para a superfície original $S$. Em particular, quando $S=S^{m}$ obtemos o operador de Laplace-Beltrami definido a seguir.

Definição 1.5.1. Seja $f$ uma função complexa definida sobre $S^{m}$. A extensão radial de $f$ é a aplicação $\tilde{f}$ dada por $\tilde{f}(x):=f(x /\|x\|), x \in \mathbb{R}_{*}^{m+1}$.

Definição 1.5.2. Seja $r$ um inteiro não negativo. Uma aplicação $f: S^{m} \rightarrow \mathbb{C}$ é $r$ vezes diferenciável (respectivamente, continuamente diferenciável), se $\tilde{f}$ ér vezes diferenciável (respectivamente, continuamente diferenciável) em $\mathbb{R}_{*}^{m+1}$. Nestas condições, para cada multi-índice $\alpha$ satisfazendo $|\alpha| \leq r$, definimos $D^{\alpha} f:=\left.\left(D^{\alpha} \tilde{f}\right)\right|_{S^{m}}$.

O conjunto das funções complexas definidas sobre $S^{m}$ que são $r$ vezes continuamente diferenciáveis no sentido descrito acima é denotado por $C^{r}\left(S^{m}\right)$. 
Definição 1.5.3. Se $f: S^{m} \rightarrow \mathbb{C}$ é uma aplicação duas vezes diferenciável, então a expressão

$$
\Delta_{m} f:=\left.(\Delta \tilde{f})\right|_{S^{m}}
$$

está bem definida. O operador diferencial $\Delta_{m}$ é denominado operador de LaplaceBeltrami. Indutivamente, escrevemos $\Delta_{m}^{1}=\Delta_{m}$ e $\Delta_{m}^{r} f=\Delta_{m} \Delta_{m}^{r-1}$, para $r=2,3, \ldots$

A seguir, apresentamos um teorema que trata da relação existente entre o operador de Laplace-Beltrami e os harmônicos esféricos. Segundo este resultado, os espaços de harmônicos esféricos estão contidos em subespaços vetoriais de $L^{2}\left(S^{m}\right)$ que são autoespaços com relação a $\Delta_{m}$.

Teorema 1.5.4. Se $f$ pertence a $\mathcal{H}_{n}^{m+1}$, então $\Delta_{m} f=-n(n+m-1) f$.

Finalizamos a seção descrevendo como pretendemos derivar núcleos sobre $S^{m}$. Para multi-índices $\alpha$ e $\beta$ em $\mathbb{Z}_{+}^{n}$, usamos os símbolos $D_{y}^{\beta} D_{x}^{\alpha}$ para indicar a derivada de ordem $\alpha$ com respeito à variável $x$ seguida pela derivada de ordem $\beta$ com respeito à variável $y$. Em particular, para um núcleo $K$ com domínio $S^{m} \times S^{m}$, escrevemos

$$
D_{y}^{\beta} D_{x}^{\alpha} K=\left.\left(D_{y}^{\beta} D_{x}^{\alpha} \widetilde{K}\right)\right|_{S^{m} \times S^{m}}
$$

onde $\widetilde{K}$ é obtido de $K$ após uma expansão radial nas duas variáveis para $\mathbb{R}_{*}^{m+1}$. Para que $D_{y}^{\beta} D_{x}^{\alpha} K$ exista, é necessário que a derivada $D_{y}^{\beta} D_{x}^{\alpha} \widetilde{K}$ exista em $\mathbb{R}_{*}^{m+1} \times \mathbb{R}_{*}^{m+1}$. Continuidade de $D_{y}^{\beta} D_{x}^{\alpha} K$ significa continuidade de $D_{y}^{\beta} D_{x}^{\alpha} \widetilde{K}$ em $\mathbb{R}_{*}^{m+1} \times \mathbb{R}_{*}^{m+1}$ e assim por diante. A fórmula $D_{y}^{\alpha} D_{x}^{\beta} K=D_{x}^{\beta} D_{y}^{\alpha} K$ é verdadeira para $K$ suficientemente suave, o que sempre ocorre em nosso contexto. Utilizamos a seguinte notação condensada para a derivação no sentido usual: $D^{\alpha, \beta}:=D_{x}^{\alpha} D_{y}^{\beta}$.

Para tornar mais claras algumas informações, introduzimos a seguinte nomenclatura: se $r$ é um inteiro não negativo, dizemos que um núcleo $K: S^{m} \times S^{m} \rightarrow \mathbb{C}$ é $(s, t)$-diferenciável se a derivada $D^{\alpha, \beta} K$ existe e é contínua para $|\alpha| \leq s$ e $|\beta| \leq t$. O espaço de tais núcleos é denotado por $C^{s, t}\left(S^{m} \times S^{m}\right)$.

De forma análoga ao que acabamos de descrever, o símbolo $\Delta_{m}^{x}$ (respectivamente, $\left.\Delta_{m}^{y}\right)$ é usado para representar a ação do operador de Laplace-Beltrami com respeito à variável $x$ (respectivamente, $y$ ), enquanto mantém-se $y$ (respectivamente, $x$ ) fixado. Não há dificuldade em aceitar que

$$
\Delta_{m}^{y} \Delta_{m}^{x} K=\Delta_{m}^{x} \Delta_{m}^{y} K, \quad K \in C^{2,2}\left(S^{m} \times S^{m}\right),
$$

enquanto um argumento indutivo leva-nos a

$$
\left(\Delta_{m}^{y}\right)^{s}\left(\Delta_{m}^{x}\right)^{s} K=\left(\Delta_{m}^{y} \Delta_{m}^{x}\right)^{s} K, \quad K \in C^{2 s, 2 s}\left(S^{m} \times S^{m}\right) .
$$




\subsection{A derivada de Laplace-Beltrami}

A derivada de Laplace-Beltrami é uma variação da derivada usual sobre $S^{m}$ quando, na definição desta última, substituímos o operador translação usual pela translação esférica

$$
T_{\epsilon}^{m}(f)(x):=\frac{1}{\sigma_{m-1}\left(1-\epsilon^{2}\right)^{(m-1) / 2}} \int_{x \cdot y=\epsilon} f(y) d y, \quad x \in S^{m} .
$$

Aqui, $\epsilon \in(-1,1)$ e $d y$ denota o elemento de medida do aro $\left\{y \in S^{m}: x \cdot y=\epsilon\right\}$ da calota esférica $\left\{y \in S^{m}: x \cdot y \geq \epsilon\right\}$. Escrevendo $\Delta_{\epsilon}:=I-T_{\epsilon}^{m}$, onde $I$ denota o operador identidade, dizemos que uma função $f \in L^{2}\left(S^{m}\right)$ é diferenciável no sentido de Laplace-Beltrami se existir $\mathcal{D} f \in L^{2}\left(S^{m}\right)$ tal que

$$
\lim _{\epsilon \rightarrow 1^{-}}\left\|(1-\epsilon)^{-1} \Delta_{\epsilon}(f)-\mathcal{D} f\right\|_{2}=0 .
$$

A função $\mathcal{D} f$ é chamada de derivada de Laplace-Beltrami de $f$. Derivadas de ordens superiores são definidas recursivamente pelas fórmulas $\mathcal{D}^{1}=\mathcal{D}$ e

$$
\mathcal{D}^{r}:=\mathcal{D}^{1} \circ \mathcal{D}^{r-1}, \quad r=2,3, \ldots
$$

O espaço de todas a funções complexas sobre $S^{m}$ que são diferenciáveis até a ordem $r$ no sentido recém definido será denotado por $W_{2}^{r}$.

O operador $\mathcal{D}^{r}$ é um operador multiplicativo no sentido que passamos a descrever. O espaço $\mathcal{H}_{n}^{m+1}$ de todos os harmônicos esféricos de $n$-ésimo grau em $m+1$ variáveis é um subconjunto de $W_{2}^{r}$ e

$$
\mathcal{D}^{r} Y=\frac{n^{r}(n+m-1)^{r}}{m^{r}} Y, \quad Y \in \mathcal{H}_{n}^{m+1} .
$$

Ele age como um operador autoadjunto sobre elementos de $W_{2}^{r}$, isto é,

$$
\left\langle\mathcal{D}^{r} f, \bar{g}\right\rangle_{2}=\left\langle f, \overline{\mathcal{D}^{r} g}\right\rangle_{2}, \quad f, g \in W_{2}^{r} .
$$

Para mais informações sobre a derivada de Laplace-Beltrami, indicamos ao leitor as referências [54, 66] e outras lá mencionadas.

A ação da derivada de Laplace-Beltrami sobre núcleos é feita separadamente, mantendo fixa uma variável enquanto deriva-se com respeito a outra. O símbolo $\mathcal{D}_{y}^{r} K$ indica a derivada de ordem $r$ de um núcleo $K$ com respeito à variável $y$ (da mesma forma definimos a derivada com respeito à variável $x$, que não será utilizada neste texto).

Seguimos mostrando a relação existente entre a derivada usual e a de LaplaceBeltrami. Uma forma de ver esta conexão é considerando o operador projeção esférica $\mathcal{Y}_{n}: L^{2}\left(S^{m}\right) \rightarrow L^{2}\left(S^{m}\right)$ dado por

$$
\mathcal{Y}_{n}(f)(x):=\frac{N(m, n)}{\sigma_{m}} \int_{S^{m}} P_{n}^{m}(x \cdot y) f(y) d \sigma_{m}(y), \quad x \in S^{m}, \quad n=0,1, \ldots
$$


Como se sabe,

$$
\mathcal{Y}_{n}\left(\Delta_{m} p\right)=-n(n+m-1) \mathcal{Y}_{n}(p), \quad p \in \mathcal{H}_{n}^{m+1},
$$

enquanto alguns cálculos revelam que

$$
m \mathcal{Y}_{n}(\mathcal{D} f)=n(n+m-1) \mathcal{Y}_{n}(f), \quad f \in W_{2}^{1} .
$$

Assim, não é surpresa que o próximo resultado seja válido.

Teorema 1.6.1. Seja $f \in L^{2}\left(S^{m}\right)$. Se existe $g \in C^{2 r}\left(S^{m}\right)$ tal que $f=g$ a.e., então $\mathcal{D}^{r} f=m^{-r}\left(-\Delta_{m}\right)^{r} g$.

Demonstração: Se $g \in C^{2 r}\left(S^{m}\right)$ e $f=g$ q.s., definimos $g_{k}:=\sum_{n=0}^{k} \mathcal{Y}_{n}(g), k=$ $1,2, \ldots$ Como o espaço $W_{2}^{1}$ contém todos os espaços $\mathcal{H}_{n}^{m+1}$, segue que $\left\{g_{k}\right\} \subset W_{2}^{1}$. Além disso, vemos que

$$
\lim _{k \rightarrow \infty}\left\|g_{k}-g\right\|_{2}=\lim _{k \rightarrow \infty}\left\|\left(-\Delta_{m}\right)^{r} g_{k}-\left(-\Delta_{m}\right)^{r} g\right\|_{2}=0 .
$$

Por outro lado,

$$
\sum_{n=0}^{l}\left(-\Delta_{m}\right)^{r} \mathcal{Y}_{n}(g)=(m)^{r} \sum_{n=0}^{k} \mathcal{D}^{r} \mathcal{Y}_{n}(g)=(m)^{r} \mathcal{D}^{r} \sum_{n=0}^{l} \mathcal{Y}_{n}(g)=(m)^{r} \mathcal{D}^{r} g_{k} .
$$

Desta forma, deduzimos que

$$
\lim _{k \rightarrow \infty}\left\|\mathcal{D}^{r} g_{k}-(m)^{-r}\left(-\Delta_{m}\right)^{r} g\right\|_{2}=0 .
$$

Como $\mathcal{D}^{r}$ é um operador fechado, concluímos que $g \in W_{2}^{r}$ e $\mathcal{D}^{r} g=(m)^{-r}\left(-\Delta_{m}\right)^{r} g$. Finalmente, podemos afirmar que $\mathcal{D}^{r} f$ existe e $\mathcal{D}^{r} f=\mathcal{D}^{r} g$ em $L^{2}\left(S^{m}\right)$.

Como o Teorema 1.6.1 estabelece a ponte entre a derivada de Laplace-Beltrami e a derivada usual, registramos sua versão para núcleos.

Teorema 1.6.2. Seja $r$ um inteiro não negativo. Se $K \in C^{2 r, 2 r}\left(S^{m} \times S^{m}\right)$, então

$$
\mathcal{D}^{s, t} K=(-m)^{-(s+t)}\left(\Delta_{m}^{x}\right)^{s}\left(\Delta_{m}^{y}\right)^{t} K, \quad s, t \leq r .
$$

\subsection{A integral de Laplace-Beltrami}

Nesta seção estudamos um operador integral que age como uma inversa para a derivada de Laplace-Beltrami. Suas potências surgem quase que naturalmente em decomposições de $\mathcal{K}$ no caso em que o núcleo gerador $K$ satisfaz hipóteses de suavidade definidas via derivada de Laplace-Beltrami. Por esta razão, este operador integral entra nas demonstrações dos principais teoremas da tese. 
O operador integral de Laplace-Beltrami é a única aplicação linear $J: L^{2}\left(S^{m}\right) \rightarrow$ $L^{2}\left(S^{m}\right)$ definida pelas condições $J 1=1 \mathrm{e}$

$$
J Y=\frac{m}{n(n+m-1)} Y, \quad Y \in \mathcal{H}_{n}^{m+1}, \quad n=1,2, \ldots .
$$

A ação inversa a que nos referimos é no sentido de que

$$
\mathcal{D} J f=J \mathcal{D} f=f, \quad f \in \oplus_{n=1}^{\infty} \mathcal{H}_{n}^{m+1} .
$$

Também podemos definir este operador via convolução esférica, começando com a aplicação $F:(-1,1) \rightarrow \mathbb{R}$ dada por

$$
F(t)=m \int_{-1}^{t}\left(1-s^{2}\right)^{-m / 2} \int_{-1}^{s} d w_{m}(u) d s, \quad t \in(-1,1),
$$

onde $d w_{m}(u):=\left(1-u^{2}\right)^{(m-2) / 2} d u$. Poucos cálculos revelam que $F \in L^{1}\left([-1,1], w_{m}\right)$, enquanto a fórmula

$$
\mathcal{L}(t):=F(t)+1-\|F\|_{1, m}, \quad t \in(-1,1),
$$

define um elemento normalizado $\mathcal{L}$ de $L^{1}\left([-1,1], w_{m}\right)$ com o primeiro coeficiente de Fourier-Legendre igual a 1 . Aqui, $\|\cdot\|_{1, m}$ indica a norma usual em $L^{1}\left([-1,1], w_{m}\right)$. Além disso, pode-se provar que

$$
J f(x)=\int_{S^{m}} \mathcal{L}(x \cdot y) f(y) d \sigma_{m}(y), \quad x \in S^{m}, \quad f \in L^{2}\left(S^{m}\right) .
$$

Esta fórmula mostra que $J f$ coincide com a convolução esférica $\mathcal{L} * f$ de $\mathcal{L}$ e $f$.

As potências de $J$ são definidas recursivamente: $J^{0}$ é o operador identidade, enquanto $J^{r}:=J \circ J^{r-1}, r=1,2, \ldots$ Também é fácil deduzir a fórmula

$$
\left\langle J^{r} f, \bar{g}\right\rangle_{2}=\left\langle f, \overline{J^{r} g}\right\rangle_{2}, \quad f, g \in L^{2}\left(S^{m}\right),
$$

que implica a autoadjunticidade de $J^{r}$ com relação ao produto escalar usual de $L^{2}\left(S^{m}\right)$. Todos estes fatos estão provados em [54, 66]. O Teorema 3.1 a seguir descreve uma propriedade que não conseguimos encontrar justificada. Logo, dispomos uma demonstração.

Teorema 1.7.1. O operador $J^{r}$ é compacto.

Demonstração: Fixamos $r$ e uma base ortonormal $\left\{Y_{n, k}: k=1,2, \ldots N(m, n)\right\}$ de $\mathcal{H}_{n}^{m+1}, n=0,1, \ldots$ Considere uma função $f$ em $L^{2}\left(S^{m}\right)$ juntamente com sua expansão 
em harmônicos esféricos condensada $f \sim \sum_{n=0}^{\infty} \Pi_{n}(f)$. Podemos usar as identidades 1.7.6 e 1.7.1 para obter

$$
\Pi_{n}\left(J^{r} f\right)=\frac{m^{r}}{n^{r}(n+m-1)^{r}} \Pi_{n}(f), \quad n=0,1, \ldots
$$

Como o espaço dos operadores compactos sobre $L^{2}\left(S^{m}\right)$ é um subconjunto fechado do espaço de todos os operadores lineares sobre $L^{2}\left(S^{m}\right)$ com respeito à norma de operadores, a demonstração estará completa tão logo provarmos que a série

$$
\sum_{n=0}^{\infty} \frac{m^{r}}{n^{r}(n+m-1)^{r}} \Pi_{n}(f)
$$

converge para $J^{r} f$ na norma de $L^{2}\left(S^{m}\right)$. Alguns cálculos produzem as desigualdades

$$
\begin{aligned}
\left\|J^{r} f-\sum_{n=0}^{l} \frac{m^{r}}{n^{r}(n+m-1)^{r}} \Pi_{n}(f)\right\|_{2} & \leq \sum_{n=l+1}^{\infty} \frac{m^{r}}{n^{r}(n+m-1)^{r}}\left\|\Pi_{n}(f)\right\|_{2} \\
& \leq \sum_{n=l+1}^{\infty} \frac{m^{r}}{n^{r}(n+m-1)^{r}}\|f\|_{2} .
\end{aligned}
$$

Depois, usamos a desigualdade $n^{2 r} \leq n^{r}(n+m-1)^{r}$ e, finalmente, vemos que

$$
\left\|J^{r} f-\sum_{n=0}^{l} \frac{m^{r}}{n^{r}(n+m-1)^{r}} \Pi_{n}(f)\right\|_{2} \leq m^{r}\|f\|_{2} \sum_{n=l+1}^{\infty} n^{-2 r} .
$$

Claramente, esta última série aproxima-se de 0 quando $l \rightarrow \infty$. 


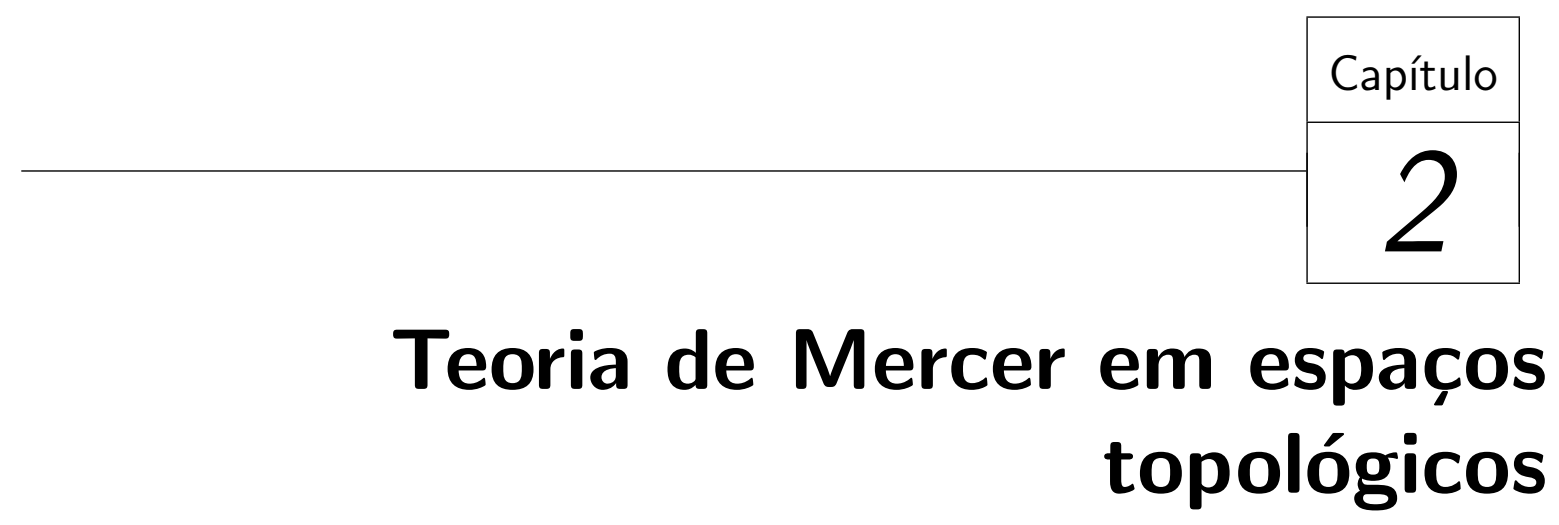

Neste capítulo estudamos operadores integrais sobre $L^{2}(X, \sigma)$ no caso em que $X$ é um espaço topológico primeiro-enumerável e $\sigma$ é uma medida de Borel não degenerada. Estabelecemos condições que garantem a validade de vários resultados associados ao Teorema de Mercer, mesmo sem exigir do espaço $X$ compacidade ou existência de métrica. Tomamos como hipótese básica a $L^{2}$-positividade definida do núcleo gerador e consideramos representações por séries para o núcleo, nuclearidade do operador e o cálculo do traço do operador integral por meio de uma fórmula de integração.

\subsection{Representação em forma de série para os núcleos geradores}

Começamos apresentando uma terminologia básica. Se $X$ é um conjunto não vazio, escrevemos $\Delta_{X}$ para denotar a diagonal do conjunto $X \times X$, isto é,

$$
\Delta_{X}:=\{(x, x): x \in X\}
$$

Neste capítulo, $X$ representa um espaço topológico que munimos com uma medida de Borel não degenerada $\mu$.

O conteúdo do lema a seguir deve ser conhecido, mas disponibilizamos uma demonstração devido à dificuldade de encontrá-lo provado em publicações conhecidas. 
Lema 2.1.1. Se $K \in L^{2}(X \times X, \mu \times \mu)$ é hermitiano e $f \in L^{2}(X, \mu)$, então

$$
\int_{X} \int_{X} K(x, y) f(x) \overline{f(y)} d \mu(x) d \mu(y)=\int_{X} \int_{X} \operatorname{Re}(K(x, y) f(x) \overline{f(y)}) d \mu(x) d \mu(y) .
$$

Demonstração: Segue da desigualdade de Hölder (Teorema 1.2.4) que a aplicação $(x, y) \in X \times X \mapsto K(x, y) f(x) \overline{f(y)}$ é integrável, enquanto que alguns cálculos mostram que

$$
\int_{X} \int_{X} K(x, y) f(x) \overline{f(y)} d \mu(x) d \mu(y)=\int_{X}\left(\int_{X} K(y, x) f(y) d \mu(y)\right) \overline{f(x)} d \mu(y) .
$$

Por outro lado, podemos mostrar que as aplicações $x \in X \mapsto K(x, y) f(x)$ e $x \in X \mapsto$ $\int_{X} K(x, y) f(x) d \mu(x)$ são integráveis para quase todo $y \in X$. Logo, pelo Teorema de Fubini, temos

$$
\overline{\int_{X} \int_{X} K(x, y) f(x) \overline{f(y)} d \mu(x) d \mu(y)}=\int_{X}\left(\int_{X} K(y, x) f(y) d \mu(y)\right) \overline{f(x)} d \mu(x) .
$$

Assim, vemos que

$$
\int_{X} \int_{X} \operatorname{Im}(K(x, y) f(x) \overline{f(y)}) d \mu(x) d \mu(y)=0,
$$

o que completa a demonstração.

No que segue, usamos o símbolo $\chi_{A}$ para denotar a função característica de um subconjunto $A$ de $X$.

Lema 2.1.2. Se $K$ é $L^{2}(X, \mu)$-positivo definido e contínuo em $\Delta_{X}$, então $K(x, x) \in \mathbb{R}$, $x \in X$.

Demonstração: Se $K$ é $L^{2}(X, \sigma)$-positivo definido, então $K$ é hermitiano $(\sigma \times \sigma)$ q.s. e, consequentemente, $K(x, x) \in \mathbb{R}$ q.s.. Portanto, se $\left.K\right|_{\Delta_{X}}$ é contínuo, então $K(x, x) \in \mathbb{R}, x \in X$.

Lema 2.1.3. Sejam $X$ um espaço topológico primeiro-enumerável munido com uma medida de Borel não degenerada $\mu$ e $K$ um núcleo $L^{2}(X, \mu)$-positivo definido. Se $K$ é contínuo em $\Delta_{X}$, então $\left.K\right|_{\Delta_{X}}$ é não negativo.

Demonstração: Segue do Lema 2.1 .2 que $K(x, x) \in \mathbb{R}, x \in X$. Completamos a prova mostrando que, nas hipóteses do lema, esta informação somada à continuidade de $K$ em $\Delta_{X}$ implica a não negatividade de $\left.K\right|_{\Delta_{X}}$. Fixe $x_{0} \in X$. Dado $\epsilon>0$, usamos a primeira-enumerabilidade para selecionar uma coleção $\left\{V_{1}, V_{2}, \ldots\right\}$ de vizinhanças de $x_{0}$ e um inteiro positivo $n_{0}$ tal que $\left|K(x, y)-K\left(x_{0}, x_{0}\right)\right|<\epsilon$, quaisquer que sejam 
$x \in V_{n}, y \in V_{m}$ e $m, n \geq n_{0}$. Segue que $\operatorname{Re} K(x, y)<\epsilon+K\left(x_{0}, x_{0}\right)$ sob as mesmas condições. Se $K\left(x_{0}, x_{0}\right)$ fosse negativo, poderíamos escolher $\epsilon \in\left(0,-K\left(x_{0}, x_{0}\right)\right)$ e usar os argumentos acima para concluir que

$$
\operatorname{Re} K(x, y)<0, \quad x, y \in V_{n}
$$

para $n$ arbitrariamente grande. Lembrando que $\mu$ é não degenerada, uma aplicação do Lema 2.1.1 é suficiente para mostrar que

$$
\begin{aligned}
\left\langle\mathcal{K}\left(\chi_{V_{n}}\right), \chi_{V_{n}}\right\rangle & =\int_{V_{n}} \int_{V_{n}} K(x, y) d \mu(x) d \mu(y) \\
& =\int_{V_{n}} \int_{V_{n}} \operatorname{Re} K(x, y) d \mu(x) d \mu(y)<0,
\end{aligned}
$$

para $n$ arbitrariamente grande, uma contradição com o fato de $K$ ser $L^{2}(X, \mu)$-positivo definido.

O Teorema 2.1.4 descreve propriedades básicas de operadores definidos por expansões somáveis com coeficientes não negativos.

Teorema 2.1.4. Sejam $(X, \mu)$ um espaço de medida e $\left\{f_{n}\right\}$ uma sequência ortonormal em $L^{2}(X, \mu)$. Suponha que existe uma sequência $\left\{a_{n}\right\}$ de números reais não negativos tais que $\left\{a_{n}\left\langle f, f_{n}\right\rangle f_{n}\right\}$ é somável em $L^{2}(X, \mu)$, para toda $f \in L^{2}(X, \mu)$. Então, a fórmula

$$
T(f)=\sum_{n=1}^{\infty} a_{n}\left\langle f, f_{n}\right\rangle f_{n}, \quad f \in L^{2}(X, \mu),
$$

define um operador linear limitado sobre $L^{2}(X, \mu)$ com as seguintes propriedades:

(i) Se $a_{n}>0$, então $f_{n}$ é um autovetor de $T$ com autovalor $a_{n}$;

(ii) $\langle T(f), f\rangle \geq 0, f \in L^{2}(X, \mu)$;

(iii) Se $T=\mathcal{K}$ para algum núcleo $K \in L^{2}(X \times X, \mu \times \mu)$, então $K$ é $L^{2}(X, \mu)$-positivo definido.

Demonstração: A linearidade de $T$ é óbvia e sua limitação segue do princípio da limitação uniforme. Não é difícil verificar a veracidade de $(i)$, e $(i i)$ segue de

$$
\langle T(f), f\rangle=\lim _{p \rightarrow \infty}\left\langle\sum_{n=1}^{p} a_{n}\left\langle f, f_{n}\right\rangle f_{n}, f\right\rangle=\lim _{p \rightarrow \infty} \sum_{n=1}^{p} a_{n}\left|\left\langle f, f_{n}\right\rangle\right|^{2} .
$$

Finalmente, (iii) segue de $(i i)$.

Se refinamos as hipóteses sobre $X$ e sobre a medida $\mu$, com um esforço extra, obtemos o próximo resultado. 
Teorema 2.1.5. Seja X um espaço topológico primeiro-enumerável munido com uma medida não degenerada $\mu$. Seja $\left\{f_{n}\right\}$ uma sequência ortonormal de funções contínuas de $L^{2}(X, \mu)$. Suponha que existe uma sequência $\left\{a_{n}\right\} \subset[0, \infty)$ tal que $\left\{a_{n}\left\langle f, f_{n}\right\rangle f_{n}\right\}$ é somável em $L^{2}(X, \mu)$, para toda $f \in L^{2}(X, \mu)$, e considere o operador $T$ definido em 2.1.4). Se $T=\mathcal{K}$, para algum $K \in L^{2}(X \times X, \mu \times \mu)$ contínuo em $\Delta_{X}$, então

$$
\sum_{n=1}^{\infty} a_{n}\left|f_{n}(x)\right|^{2} \leq K(x, x), \quad x \in X .
$$

Demonstração: Suponha que $T$ coincide com um operador integral $\mathcal{K}$ gerado por um núcleo $K \in L^{2}(X \times X, \mu \times \mu)$ e seja $p$ um inteiro positivo fixado. Devido à hipótese de somabilidade,

$$
\begin{aligned}
& \int_{X} \sum_{n=p+1}^{\infty} a_{n}\left\langle f, f_{n}\right\rangle f_{n}(x) \overline{f(x)} d \mu(x) \\
&=\sum_{n=p+1}^{\infty} a_{n}\left\langle f, f_{n}\right\rangle \int_{X} f_{n}(x) \overline{f(x)} d \mu(x), \quad f \in L^{2}(X, \mu),
\end{aligned}
$$

isto é,

$$
\int_{X} \sum_{n=p+1}^{\infty} a_{n}\left\langle f, f_{n}\right\rangle f_{n}(x) \overline{f(x)} d \mu(x)=\sum_{n=p+1}^{\infty} a_{n}\left|\left\langle f, f_{n}\right\rangle\right|^{2}, \quad f \in L^{2}(X, \mu) .
$$

Claramente, o núcleo $K_{p}$ definido por

$$
K_{p}(x, y):=K(x, y)-\sum_{n=1}^{p} a_{n} f_{n}(x) \overline{f_{n}(y)}, \quad x, y \in X,
$$

será um elemento de $L^{2}(X \times X, \mu \times \mu)$ contínuo em $\Delta_{X}$, sempre que $K$ também for. Usando (2.1.7), podemos ver que o operador integral $\mathcal{K}_{p}$ satisfaz

$$
\begin{aligned}
\left\langle\mathcal{K}_{p}(f), f\right\rangle & =\int_{X}\left[\mathcal{K}(f)(x)-\sum_{n=1}^{p} a_{n}\left\langle f, f_{n}\right\rangle f_{n}(x)\right] \overline{f(x)} d \mu(x) \\
& =\sum_{n=p+1}^{\infty} a_{n}\left|\left\langle f, f_{n}\right\rangle\right|^{2}
\end{aligned}
$$

para todo $f \in L^{2}(X, \mu)$. Em particular, $K_{p}$ é $L^{2}(X, \mu)$-positivo definido. Assim, se $K$ é contínuo em $\Delta_{X}$, uma aplicação do Lema 2.1.3 garante que $K_{p}(x, x) \geq 0, x \in X$. Como $p$ foi tomado de forma arbitrária, a desigualdade do enunciado do teorema está provada.

Estamos prontos para abordar o tema de maior interesse na teoria de Mercer, a representação de $K$ em forma de série. O Teorema 2.1.6 a seguir é o primeiro passo na direção deste objetivo. 
Teorema 2.1.6. Seja $X$ um espaço topológico primeiro-enumerável munido com uma medida não degenerada $\mu$. Seja $\left\{f_{n}\right\}$ um sequência ortonormal de funções contínuas de $L^{2}(X, \mu)$. Suponha que existe uma sequência $\left\{a_{n}\right\} \subset[0, \infty)$ tal que $\left\{a_{n}\left\langle f, f_{n}\right\rangle f_{n}\right\}$ é somável em $L^{2}(X, \mu)$, para toda $f \in L^{2}(X, \mu)$, e considere o operador $T$ definido em 2.1.4). Se $T=\mathcal{K}$ para algum $K \in L^{2}(X \times X, \mu \times \mu)$ contínuo em $\Delta_{X}$, então $\sum_{n=1}^{\infty} a_{n} f_{n}(x) \overline{f_{n}(y)}$ é absolutamente e uniformemente convergente sobre subconjuntos compactos de $X$ com respeito a uma variável, quando a outra é mantida fixa.

Demonstração: Suponha que $T=\mathcal{K}$ para algum $K \in L^{2}(X \times X, \mu \times \mu)$ contínuo em $\Delta_{X}$. Seja $x \in X$ fixado e $Y$ um subconjunto compacto de $X$. A desigualdade de Cauchy-Schwarz implica que

$$
\left|\sum_{n=p}^{q} a_{n} f_{n}(x) \overline{f_{n}(y)}\right|^{2} \leq \sup _{\zeta \in Y} K(\zeta, \zeta) \sum_{n=p}^{q} a_{n}\left|f_{n}(x)\right|^{2}, \quad y \in Y
$$

onde $1 \leq p \leq q$. Uma aplicação do critério de Cauchy para convergência uniforme, mais a desigualdade 2.1 .5 , implica que $\sum_{n=1}^{\infty} a_{n} f_{n}(x) \overline{f_{n}(y)}$ é absolutamente e uniformemente convergente em $Y$. Similarmente, se $y$ é fixado, a mesma série é absolutamente e uniformemente convergente sobre $Y$.

Se $X$ tem medida finita, o operador integral $\mathcal{K}$ gerado por um núcleo limitado e contínuo $K$ possui a propriedade adicional de que sua imagem contém apenas funções contínuas. No que diz respeito a este texto, o resultado equivalente é o que segue.

Teorema 2.1.7. Sob as mesmas hipóteses do Teorema 2.1.6, se $T=\mathcal{K}$ para algum $K \in L^{2}(X \times X, \mu \times \mu)$ contínuo em $\Delta_{X}$, então a imagem de $T$ contém somente funções continuas.

Demonstração: Fixe $f \in L^{2}(X, \mu)$ e $x \in X$. Considere uma sequência $\left\{x_{n}\right\} \subset X$ convergindo para $x$ e escreva $Y=\{x\} \cup\left\{x_{n}: n=1,2, \ldots\right\}$. Se $T=\mathcal{K}$ para algum $K \in L^{2}(X \times X, \mu \times \mu)$, a desigualdade de Cauchy-Schwarz e o Teorema 2.1.5 revelam que

$$
\begin{aligned}
\left|\sum_{n=p}^{q} a_{n}\left\langle f, f_{n}\right\rangle f_{n}(y)\right|^{2} & \leq \sum_{n=p}^{q} a_{n}\left|\left\langle f, f_{n}\right\rangle\right|^{2} \sum_{n=p}^{q} a_{n}\left|f_{n}(y)\right|^{2} \\
& \leq K(y, y) \sum_{n=p}^{q} a_{n}\left|\left\langle f, f_{n}\right\rangle\right|^{2}, \quad y \in Y .
\end{aligned}
$$


sempre que $1 \leq p \leq q$. Se, além disso, $K$ é contínua em $\Delta_{X}$, a compacidade de $Y$ permite deduzir que

$$
\begin{aligned}
\left|\sum_{n=p}^{q} a_{n}\left\langle f, f_{n}\right\rangle f_{n}(y)\right|^{2} & \leq \sup _{\zeta \in Y} K(\zeta, \zeta) \sum_{n=p}^{q} a_{n}\left|\left\langle f, f_{n}\right\rangle\right|^{2} \\
& \leq\|T\| \sup _{\zeta \in Y} K(\zeta, \zeta) \sum_{n=p}^{q}\left|\left\langle f, f_{n}\right\rangle\right|^{2}, \quad y \in X,
\end{aligned}
$$

para $1 \leq p \leq q$

A série $\sum_{n=1}^{\infty}\left|\left\langle f, f_{n}\right\rangle\right|^{2}$ é convergente pela desigualdade de Bessel. Logo, o critério de Cauchy para convergência mostra que $\sum_{n=1}^{\infty} a_{n}\left\langle f, f_{n}\right\rangle f_{n}(y)$ é uniformemente convergente, para todo $y \in Y$. Portanto, ela define uma função contínua sobre $Y$ e, em particular, $\left\{T(f)\left(x_{n}\right)\right\}$ converge para $T(f)(x)$. Como $X$ é primeiro-enumerável, isto implica a continuidade em $x$.

Outra contribuição para o círculo de idéias em torno do resultado anterior diz respeito à convergência em $L^{2}(X, \mu)$ da série aparecendo em 2.1.5. Isto pode ser obtido supondo compacidade local para $X$ e integrabilidade para $x \in X \mapsto K(x, x)$, como mostramos no próximo resultado.

Teorema 2.1.8. Seja X um espaço topológico primeiro-enumerável, localmente compacto e munido com uma medida não degenerada $\mu$. Seja $\left\{f_{n}\right\}$ uma sequência ortonormal de funções contínuas de $L^{2}(X, \mu)$. Suponha que existe uma sequência $\left\{a_{n}\right\} \subset[0, \infty)$ tal que $\left\{a_{n}\left\langle f, f_{n}\right\rangle f_{n}\right\}$ é somável em $L^{2}(X, \mu)$, qualquer que seja $f \in L^{2}(X, \mu)$, e considere o operator $T$ definido em (2.1.4). Se $T=\mathcal{K}$, para algum $K \in L^{2}(X \times X, \mu \times \mu)$ contínuo em $\Delta_{X}$, e $x \in X \mapsto K(x, x)$ é integrável, então $\sum_{n=1}^{\infty} a_{n}\left|f_{n}(x)\right|^{2}$ converge em $L^{2}(X, \mu)$ para a função $x \in X \rightarrow K(x, x)$.

Demonstração: Suponha que $T=\mathcal{K}$, com $K \in L^{2}(X \times X, \mu \times \mu)$ contínuo em $\Delta_{X}$. Lembrando de 2.1.5), vemos que

$$
\sum_{n=1}^{\infty}\left|a_{n} f_{n}(x)\right|^{2} \leq\|T\| \sum_{n=1}^{\infty} a_{n}\left|f_{n}(x)\right|^{2} \leq\|T\| K(x, x), \quad x \in X .
$$

Devido ao Teorema 1.3 .4 (de Riesz-Fisher) concluímos que, para todo $x \in X$, a soma $\sum_{n=1}^{\infty} a_{n} f_{n}(x) \overline{f_{n}}$ converge em $L^{2}(X, \mu)$ para uma função $K_{x} \in L^{2}(X, \mu)$. Como consequência,

$$
\begin{aligned}
\int_{X} K_{x}(y) f(y) d \mu(y) & =\sum_{n=1}^{\infty} a_{n}\left\langle f, f_{n}\right\rangle f_{n}(x) \\
& =T(f)(x)=\mathcal{K}(f)(x) \\
& =\int_{X} K(x, y) f(y) d \mu(y), \quad x \in X
\end{aligned}
$$


qualquer que seja $f \in L^{2}(X, \mu)$. Em particular, $K_{x}=K(x, \cdot)$ q.s.. Como as conclusões do Teorema 2.1.6 são válidas, invocamos a compacidade local de $X$ para selecionar, para cada $y \in X$, um subconjunto compacto $U_{y}$ de $X$ onde a convergência de $\sum_{n=1}^{\infty} a_{n} f_{n}(x) \overline{f_{n}}$ para $K_{x}$ é uniforme. Assim, $K_{x}$ é contínua em $U_{y}$, qualquer que seja $y \in X$. Em particular, a igualdade $K_{x}=K(x, \cdot)$ é verdadeira para todo $x \in X$. Desta forma, vemos que

$$
K(x, x)=K_{x}(x)=\sum_{n=1}^{\infty} a_{n}\left|f_{n}(x)\right|^{2}, \quad x \in X \text { q.s.. }
$$

Como

$$
\sum_{n=1}^{N} a_{n}\left|f_{n}(x)\right|^{2} \leq K(x, x), \quad x \in X \text { q.s. }
$$

a continuidade das funções envolvidas implica que a desigualdade é sempre verdadeira. Consequentemente, se $x \in X \mapsto K(x, x)$ é integrável, então obtemos um limite uniforme em $L^{1}(X, \mu)$ para a sequência de somas parciais acima. Isto é suficiente para garantir a convergência da série para $x \in X \mapsto K(x, x)$ em $L^{2}(X, \mu)$.

No último resultado da seção lidamos com a convergência da série $\sum_{n=1}^{\infty} a_{n} f_{n}(x) \overline{f_{n}(y)}$ no caso em que ambas as variáveis $x$ e $y$ percorrem $X$. A integrabilidade de $x \in X \mapsto$ $K(x, x)$ não é mais necessária.

Teorema 2.1.9. Seja X um espaço topológico primeiro-enumerável, localmente compacto e munido com uma medida não degenerada $\mu$. Seja $\left\{f_{n}\right\}$ uma sequência ortonormal de funções contínuas de $L^{2}(X, \mu)$. Suponha que existe uma sequência $\left\{a_{n}\right\} \subset[0, \infty)$ tal que $\left\{a_{n}\left\langle f, f_{n}\right\rangle f_{n}\right\}$ é somável em $L^{2}(X, \mu)$, para toda $f \in L^{2}(X, \mu)$, e considere o operador $T$ definido em (2.1.4). Se $T=\mathcal{K}$ para algum $K \in L^{2}(X \times X, \mu \times \mu)$ contínuo em $\Delta_{X}$, então a série $\sum_{n=1}^{\infty} a_{n} f_{n}(x) \overline{f_{n}(y)}$ converge absolutamente e uniformemente sobre subconjuntos compactos de $X \times X$.

Demonstração: A Fórmula 2.1.15 e o Teorema de Dini para espaços topológicos compactos implicam que a convergência de $\sum_{n=1}^{\infty} a_{n}\left|f_{n}(x)\right|^{2}$ para $K(x, x)$ é uniforme sobre subconjuntos compactos de $X$. Como a desigualdade de Cauchy-Schwarz implica que

$$
\left|\sum_{n=p}^{q} a_{n} f_{n}(x) \overline{f_{n}(y)}\right|^{2} \leq \sum_{n=p}^{q} a_{n}\left|f_{n}(x)\right|^{2} \sum_{n=p}^{q} a_{n}\left|f_{n}(y)\right|^{2}, \quad x, y \in X,
$$

$1 \leq p \leq q$, então são consequências automáticas as convergências uniforme e absoluta de $\sum_{n=1}^{\infty} a_{n} f_{n}(x) \overline{f_{n}(y)}$ sobre subconjuntos compactos de $X \times X$. 


\subsection{Núcleos geradores suaves}

A situação descrita nos resultados da seção anterior torna-se verdadeira sempre que o núcleo gerador do operador integral for suave no sentido motivado pelo lema abaixo. O último resultado da seção apresenta um conjunto de hipóteses sob o qual o núcleo gerador é automaticamente suave.

Como já observamos anteriormente, se $(X, \mu)$ é um espaço de medida e $K$ é $L^{2}(X, \mu)$ positivo definido, então o operador integral $\mathcal{K}$ correspondente é compacto e autoadjunto e o Teorema de Hilbert-Schmidt pode ser aplicado. Assim, vemos que existe uma sequência ortonormal $\left\{f_{n}\right\}$ em $L^{2}(X, \mu)$ e uma sequência não crescente $\left\{a_{n}\right\} \subset$ $[0, \infty)$ tais que se $f \in L^{2}(X, \mu)$, então a série $\sum_{n=1}^{\infty} a_{n}\left\langle f, f_{n}\right\rangle f_{n}$ converge em $L^{2}(X, \mu)$ para $\mathcal{K}(f)$. Se $L^{2}(X, \mu)$ é separável, então a sequência pode ser considerada um conjunto completo. O Lema 2.2.1 abaixo complementa a informação dada por este resultado quando uma hipótese de continuidade é adicionada ao contexto.

Lemma 2.2.1. Sejam $(X, \mu)$ um espaço de medida e $K$ um núcleo $L^{2}(X, \mu)$-positivo definido. Se $y \in X \mapsto K(x, y)$ pertence a $L^{2}(X, \mu)$, para todo $x \in X$, e $x \in X \mapsto$ $K(x, \cdot) \in L^{2}(X, \mu)$ é contínua, então as funções $f_{n}$ acima são contínuas quando $a_{n}>0$.

Demonstração: Como $\mathcal{K}\left(f_{n}\right)=a_{n} f_{n}, n=1,2, \ldots$, basta mostrar que a imagem de $\mathcal{K}$ contém somente funções contínuas. Mas, isto segue da desigualdade

$$
|\mathcal{K}(f)(x)-\mathcal{K}(f)(y)| \leq\|K(x, \cdot)-K(y, \cdot)\|_{2}\|f\|_{2}, \quad x, y \in X
$$

$f \in L^{2}(X, \mu)$, e das hipóteses do lema.

Definição 2.2.1. Dizemos que um núcleo $K: X \times X \rightarrow \mathbb{C} e ́$ suave se as três condições estiverem satisfeitas:

(i) $K$ é contínuo em $\Delta_{X}$;

(ii) Para cada $x \in X$, a função $y \in X \mapsto K(x, y)$ pertence a $L^{2}(X, \mu)$;

(iii) A função $x \in X \mapsto K(x, \cdot) \in L^{2}(X, \mu)$ é contínua.

Os Teoremas 2.1.6 e 2.1.9 podem ser re-enunciados da seguinte forma quando esta noção de suavidade é adicionada ao contexto.

Teorema 2.2.2. Sejam $X$ um espaço topológico primeiro-enumerável munido com uma medida não degenerada $\mu$ e $K$ um núcleo $L^{2}(X, \mu)$-positivo definido e suave. Então, valem as conclusões do Lema 2.2.1 e, além disso, a série $\sum_{n=1}^{\infty} a_{n} f_{n}(x) \overline{f_{n}(y)}$ converge absolutamente e uniformemente sobre subconjuntos compactos de $X$ com respeito a uma variável, quando a outra é fixada. 
Teorema 2.2.3. Seja X um espaço topológico primeiro-enumerável e localmente compacto, munido com uma medida não degenerada $\mu$. Seja $K$ um núcleo $L^{2}(X, \mu)$-positivo definido e suave. Então, valem as conclusões do Lema 2.2.1 e, além disso, a série $\sum_{n=1}^{\infty} a_{n} f_{n}(x) \overline{f_{n}(y)}$ converge absolutamente e uniformemente sobre subconjuntos compactos de $X \times X$.

No que segue, pretendemos obter suavidade do núcleo gerador a partir da hipótese de positividade definida, ainda mantendo o ambiente não métrico adotado até agora. Um conjunto de hipóteses que permite tal implicação é descrito no Teorema 2.2.4 a seguir.

Teorema 2.2.4. Seja $X$ um espaço topológico primeiro-enumerável e localmente compacto, munido com uma medida não degenerada e localmente finita $\mu$. Se $K$ é um núcleo contínuo e $L^{2}(X, \mu)$-positivo definido, então

$$
|K(x, y)|^{2} \leq K(x, x) K(y, y), \quad x, y \in X .
$$

Demonstração: Pretendemos aplicar o Teorema 2.2.2. Seja $K$ um núcleo contínuo e $L^{2}(X, \mu)$-positivo definido. Primeiro, fixamos $x_{0} \in X$. Se $y \in X$, podemos usar a compacidade local de $X$ para escolher vizinhanças compactas de $x_{0}$ e $y$ em $X$ tais que $\mu$ é não degenerada na união $X_{y}$ delas, quando munimos $X_{y}$ com a topologia induzida de $X$. Neste caso, também temos que $X_{y}$ é primeiro-enumerável. Desta forma, o subespaço topológico $X_{y}$ munido com $\mu$ satisfaz as hipóteses do Teorema 2.2.2. No próximo passo, pretendemos mostrar que a restrição $K_{y}$ de $K$ a $X_{y} \times X_{y}$ é suave. Como $K$ é contínuo, então também o são suas restrições a $X_{y} \times X_{y}$ e a $\Delta_{X}$. Em particular, vale a condição (i) na definição de suavidade. Como $X_{y} \times X_{y}$ é compacto em $X \times X$ e $K_{y}$ é contínua em $X_{y} \times X_{y}$, existe $M$ positivo tal que $\left|K_{y}(x, w)\right| \leq M, x, w \in X_{y}$. A finitude local de $\mu$ agora implica que

$$
\int_{X_{y}}\left|K_{y}(x, w)\right|^{2} d \mu(w) \leq M^{2} \mu\left(X_{y}\right)<\infty .
$$

Segue daí a condição (ii) para suavidade. Para lidar com a condição (iii), seja $\left\{x_{n}\right\}$ uma sequência em $X_{y}$ convergindo para $\bar{x}$. Pela continuidade,

$$
\lim _{n \rightarrow \infty}\left|K\left(x_{n}, w\right)-K(\bar{x}, w)\right|=0, \quad w \in X_{y}
$$

Como

$$
\left|K\left(x_{n}, w\right)-K(\bar{x}, w)\right|^{2} \leq 4 M^{2} \in L^{1}\left(X_{y}, \mu\right),
$$

fica justificado o uso do Teorema da Convergência Dominada para obter

$$
\lim _{n \rightarrow \infty} \int_{X_{y}}\left|K\left(x_{n}, w\right)-K(\bar{x}, w)\right|^{2} d \mu(w)=0 .
$$


Como $X_{y}$ é primeiro-enumerável, isto basta para provar a condição (iii). Para provar que $K_{y}$ é $L^{2}\left(X_{y}, \mu\right)$-positivo definido, tomamos $f \in L^{2}\left(X_{y}, \mu\right)$, definimos $f$ fora de $X_{y}$ como sendo zero, e obtemos $\tilde{f} \in L^{2}(X, \mu)$ tal que

$$
\begin{aligned}
& \int_{X_{y}} \int_{X_{y}} K_{y}(x, w) f(x) \overline{f(w)} d \mu(w) d \mu(x) \\
&=\int_{X} \int_{X} K(x, w) \tilde{f}(x) \overline{\tilde{f}(w)} d \mu(w) d \mu(x) .
\end{aligned}
$$

Desta forma, a $L^{2}(X, \mu)$-positividade definida de $K$ implica

$$
\int_{X_{y}} \int_{X_{y}} K_{y}(x, w) f(x) \overline{f(w)} d \mu(w) d \mu(x) \geq 0 .
$$

Agora, podemos tomar uma sequência ortonormal $\left\{\phi_{n}\right\}$ de funções contínuas em $X_{y}$ e uma sequência não crescente de números não negativos $\left\{a_{n}\right\}$ tais que

$$
\sum_{n=1}^{\infty} a_{n}\left\langle f, \phi_{n}\right\rangle \phi_{n}=\mathcal{K}_{y}(f), \quad f \in L^{2}\left(X_{y}, \mu\right) .
$$

Uma aplicação do Teorema 2.2 .2 e a utilização de argumentos usados na demonstração do Teorema 2.1.8 permitem deduzir que $\sum_{n=1}^{\infty} a_{n} \phi_{n}\left(x_{0}\right) \overline{\phi_{n}(w)}$ converge uniformemente para $K\left(x_{0}, w\right)$ em $X_{y}$. Usando a desigualdade de Cauchy-Schwarz, obtemos

$$
\begin{aligned}
\left|K\left(x_{0}, y\right)\right|^{2}=\left|\sum_{n=1}^{\infty} a_{n} \phi_{n}\left(x_{0}\right) \overline{\phi_{n}(y)}\right|^{2} & \\
& \leq \sum_{n=1}^{\infty} a_{n}\left|\phi_{n}\left(x_{0}\right)\right|^{2} \sum_{n=1}^{\infty} a_{n}\left|\phi_{n}(y)\right|^{2}=K\left(x_{0}, x_{0}\right) K(y, y) .
\end{aligned}
$$

Como $x_{0}$ e $y$ foram tomados arbitrariamente, a prova está completa.

Um bônus extraído da demonstração do Teorema 2.2.4 é uma configuração sob a qual a suavidade do núcleo gerador segue da $L^{2}(X, \mu)$-positividade definida.

Teorema 2.2.5. Seja X um espaço topológico primeiro-enumerável e localmente compacto munido com uma medida não degenerada e localmente finita $\mu$. Se $K$ é um núcleo contínuo e $L^{2}(X, \mu)$-positivo definido e $x \in X \mapsto K(x, x)$ é integrável, então $K$ é suave.

Demonstração: Se $K$ é contínuo e $L^{2}(X, \mu)$-positivo definido e $x \in X \mapsto K(x, x)$ é integrável, a fórmula deduzida no teorema anterior implica que

$$
\int_{X}|K(x, y)|^{2} d \mu(y)<\infty, \quad x \in X
$$


Isto equivale à condição (ii) na definição de suavidade. Para provar a continuidade de $x \in X \mapsto K(x, \cdot) \in L^{2}(X, \mu)$ em um ponto $x_{0} \in X$, tomamos uma sequência $\left\{x_{n}\right\}$ em $X$ convergindo para $x_{0}$. Como $K$ é contínuo, a sequência $\left\{K\left(x_{n}, y\right)\right\}$ converge para $K\left(x_{0}, y\right)$, para todo $y \in X$ fixado. Usando a última desigualdade da prova do Teorema 2.2 .4 , deduzimos que

$$
\begin{aligned}
\left|K\left(x_{n}, y\right)-K\left(x_{0}, y\right)\right|^{2} & \leq 2\left|K\left(x_{n}, y\right)\right|^{2}+2\left|K\left(x_{0}, y\right)\right|^{2} \\
& \leq 2 K(y, y)\left[K\left(x_{n}, x_{n}\right)+K\left(x_{0}, x_{0}\right)\right], \quad y \in X
\end{aligned}
$$

Agora, fica claro que

$$
\left|K\left(x_{n}, y\right)-K\left(x_{0}, y\right)\right| \leq 2 K(y, y) \sup \left\{K\left(x_{m}, x_{m}\right): m=0,1, \ldots\right\}, \quad y \in X
$$

Como $\left|K\left(x_{n}, \cdot\right)-K(x, \cdot)\right|^{2} \in L^{1}(X, \mu), n=1,2, \ldots, \mathrm{e} \lim _{n \rightarrow \infty}\left|K\left(x_{n}, y\right)-K(x, y)\right|^{2}=0$, o Teorema da Convergência Dominada implica a continuidade exigida na condição (iii).

\subsection{Nuclearidade}

Apresentamos agora condições necessárias para que um núcleo $L^{2}$-positivo definido seja nuclear. Além disso, obtemos uma fórmula integral para calcular o traço do operador integral gerado pelo núcleo.

Como vamos lidar com o conceito de nuclearidade, a partir de agora passamos a supor que hipóteses adicionais sobre $\mathcal{M}$ e $\mu$ foram feitas com o objetivo de tornar $L^{2}(X, \mu)$ separável. Uma opção conveniente para obter isto é supor que $\mathcal{M}$ é enumeravelmente gerado (a menos de conjuntos com medida $\mu$-nula) e $X$ é $\sigma$-finito (Teorema 1.2 .8 .

Teorema 2.3.1. Seja X um espaço topológico primeiro-enumerável e localmente compacto munido com uma medida não degenerada e localmente finita $\mu$. Se $K$ é um núcleo contínuo e $L^{2}(X, \mu)$-positivo definido e $x \in X \mapsto K(x, x)$ é integrável, então $\mathcal{K}$ é nuclear $e$

$$
\operatorname{tr}(\mathcal{K})=\int_{X} K(x, x) d \mu(x)
$$

Demonstração: Se $K$ é um núcleo contínuo e $L^{2}(X, \mu)$-positivo definido, o Teorema 2.2 .5 implica que $K$ é suave. O Lema 2.2.1 pode ser aplicado para obter a existência de uma sequência de funções contínuas ortonormal completa $\left\{f_{n}\right\}$ em $L^{2}(X, \mu)$ e uma sequência não crescente $\left\{a_{n}\right\} \subset[0, \infty)$ tais que $\sum_{n=1}^{\infty} a_{n}\left\langle f, f_{n}\right\rangle f_{n}$ é convergente para $\mathcal{K}(f)$ em $L^{2}(X, \mu)$, sempre que $f \in L^{2}(X, \mu)$. Como $\mathcal{K}$ é autoadjunto, após dispor os 
valores singulares de $\mathcal{K}$ em ordem decrescente e contando suas multiplicidades, isto é, $s_{1}(\mathcal{K}) \geq s_{2}(\mathcal{K}) \geq \ldots$, podemos deduzir que $a_{n}=s_{n}(\mathcal{K})([84$, p.204]). O Teorema 2.1.8 autoriza-nos a escrever

$$
\sum_{n=1}^{\infty} s_{n}(\mathcal{K})=\sum_{n=1}^{\infty} a_{n}\left\|f_{n}\right\|_{2}^{2}=\int_{X} K(x, x) d \mu(x),
$$

o que encerra a demonstração. 
Capítulo

\section{Núcleos Laplace-Beltrami diferenciáveis}

Neste capítulo, obtemos resultados que tratam da ação da derivada de LaplaceBeltrami sobre núcleos suficientemente suaves definidos na esfera, aqueles definidos por expansões absolutamente e uniformemente convergentes geradas por famílias de funções que são ao menos contínuas. Um dos principais resultados do capítulo descreve condições que tornam possível a troca da derivada de Laplace-Beltrami com o somatório da série que representa o núcleo. Em outro, mostramos que derivadas convenientes de núcleos positivos definidos ainda definem núcleos positivos definidos.

A idéia para o estudo destas propriedades dos núcleos positivos definidos vem de [41, 53]. Em [41], Kadota prova resultados semelhantes para núcleos reais, simétricos e positivos definidos tomando valores no quadrado $[0,1] \times[0,1]$. Em [53], Menegatto, Peron e Oliveira apresentam versões esféricas para os resultados de Kadota, no caso da diferenciabilidade usual, como apresentado na Definição 1.5.2. O Teorema 1.6.2 faz, então, a ligação entre os resultados demonstrados em [53] e os apresentados aqui.

Os núcleos com os quais pretendemos lidar possuem expansões absolutamente e uniformemente convergentes da forma

$$
K(x, y)=\sum_{k=0}^{\infty} a_{k} Y_{k}(x) \overline{Y_{k}(y)}, \quad x, y \in S^{m},
$$

onde $a_{k}>0$ para todo $k$ e $\left\{Y_{k}: k=0,1, \ldots\right\}$ é uma sequência ortonormal de funções contínuas complexas em $L^{2}\left(S^{m}\right)$. A convergência uniforme permite integrar $K$ e deduzir 
a fórmula de reprodução

$$
\sigma_{m} Y_{k}=\frac{1}{a_{k}} \int_{S^{m}} K(\cdot, y) Y_{k}(y) d \sigma_{m}(y) .
$$

Expansões como em 3.0.1 definem núcleos positivos definidos.

Usando fórmulas para derivadas parciais sobre a esfera, vemos que a composição $\Delta_{m}^{r}:=\Delta_{m} \circ \cdots \circ \Delta_{m}(r$ vezes $)$ pode ser escrita como

$$
\Delta_{m}^{r} f=\sum_{i_{1}=1}^{m+1} \sum_{i_{2}=1}^{m+1} \cdots \sum_{i_{r}=1}^{m+1} D^{2\left(e_{i_{1}}+\cdots+e_{i_{r}}\right)} f, \quad f \in C^{2 r}\left(S^{m}\right),
$$

onde os símbolos $e_{j}$ denotam os vetores de $\mathbb{R}^{m+1}$ tendo 1 na $j$-ésima componente e 0 nas demais.

Nosso passo inicial começa com a extração da diferenciabilidade de Laplace-Beltrami de $Y_{k}$ a partir da diferenciabilidade de $K$. No lema a seguir, apresentamos uma representação integral para $T_{\varepsilon}^{m}\left(Y_{k}\right)$.

Lema 3.0.1. Se $\epsilon \in(-1,1)$, então

$$
\sigma_{m} T_{\epsilon}^{m}\left(Y_{k}\right)=\frac{1}{a_{k}} \int_{S^{m}} T_{\epsilon}^{m}(K(\cdot, y)) Y_{k}(y) d \sigma_{m}(y), \quad k=1,2, \ldots
$$

Demonstração: Para cada $x \in S^{m}, k \in \mathbb{Z}_{+}$e $\varepsilon \in(-1,1)$, obtemos de 3.0 .2 que

$$
\begin{aligned}
T_{\varepsilon}^{m}\left(Y_{k}\right)(x) & =\frac{1}{\sigma_{m-1}\left(1-\epsilon^{2}\right)^{(m-1) / 2}} \int_{x \cdot w=\epsilon} \frac{\sigma_{m}^{-1}}{a_{k}} \int_{S^{m}} K(w, y) Y_{k}(y) d \sigma_{m}(y) d w \\
& =\frac{\sigma_{m}^{-1}}{a_{k}} \frac{1}{\sigma_{m-1}\left(1-\epsilon^{2}\right)^{(m-1) / 2}} \int_{x \cdot w=\epsilon} \int_{S^{m}} K(w, y) Y_{k}(y) d \sigma_{m}(y) d w
\end{aligned}
$$

O Teorema de Fubini garante que a integral dupla acima pode ser iterada. Assim, vemos que

$$
\begin{aligned}
T_{\varepsilon}^{m}\left(Y_{k}\right)(x) & =\frac{\sigma_{m}^{-1}}{a_{k}} \int_{S^{m}} \frac{1}{\sigma_{m-1}\left(1-\epsilon^{2}\right)^{(m-1) / 2}} \int_{x \cdot w=\epsilon} K(w, y) d \omega Y_{k}(y) d \sigma_{m}(y) \\
& =\frac{\sigma_{m}^{-1}}{a_{k}} \int_{S^{m}} T_{\varepsilon}^{m}(K(\cdot, y))(x) Y_{k}(y) d \sigma_{m}(y)
\end{aligned}
$$

como queríamos demonstrar.

Teorema 3.0.2. Seja s um inteiro positivo. Suponha que $\mathcal{D}_{x}^{s} K$ existe e tem quadrado integrável em $L^{2}\left(S^{m} \times S^{m}\right)$. Então, $\mathcal{D}^{s} Y_{k}$ também existe $e$

$$
\sigma_{m} \mathcal{D}^{s} Y_{k}=\frac{1}{a_{k}} \int_{S^{m}} \mathcal{D}_{x}^{s} K(\cdot, y) Y_{k}(y) d \sigma_{m}(y)
$$


Demonstração: Dispomos aqui o primeiro passo da demonstração por indução sobre $s$. Para isto é suficiente mostrar que $\lim _{\epsilon \rightarrow 1^{-}}\left\|M_{\epsilon}\right\|_{2}=0$, onde

$$
M_{\epsilon}=\frac{Y_{k}-T_{\epsilon}^{m}\left(Y_{k}\right)}{1-\epsilon}-\frac{1}{\sigma_{m} a_{k}} \int_{S^{m}} \mathcal{D}_{x} K(\cdot, y) Y_{k}(y) d \sigma_{m}(y)
$$

Lembrando de 3.0 .2 e do lema anterior, vemos que

$$
M_{\epsilon}=\frac{1}{\sigma_{m} a_{k}} \int_{S^{m}}\left(\frac{K(\cdot, y)-T_{\epsilon}^{m} K(\cdot, y)}{1-\epsilon}-\mathcal{D}_{x} K(\cdot, y)\right) Y_{k}(y) d \sigma_{m}(y) .
$$

Como $\mathcal{D}_{x}^{s} K \in L^{2}\left(S^{m} \times S^{m}\right)$, o Teorema de Fubini permite que usemos a desigualdade de Minkowski para integrais (Teorema 1.2.7) para obter

$$
\left\|M_{\epsilon}\right\|_{2} \leq \frac{1}{\sigma_{m} a_{k}} \int_{S^{m}}\left\|\frac{K(\cdot, y)-T_{\epsilon}^{m} K(\cdot, y)}{1-\epsilon}-\mathcal{D}_{x} K(\cdot, y)\right\|_{2}\left|Y_{k}(y)\right| d \sigma_{m}(y) .
$$

A fórmula do limite segue de uma aplicação do Teorema da convergência dominada. $\square$

Observação 3.0.3. Se $K$ é contínuo, $D^{\alpha, \alpha} K$ existe para algum $\alpha$ e $|2 \beta| \leq|\alpha|$, então $\mathcal{D}^{\beta, \beta} K$ existe. Este fato será usado implicitamente na demonstração do Teorema 3.2 .2 adiante.

É importante relembrar que nosso contexto permite comutar os símbolos de derivação.

\subsection{Positividade e a derivada de Laplace-Beltrami}

O resultado principal nesta seção apresenta uma fórmula que descreve a ação de certos operadores definidos por derivadas de Laplace-Beltrami sobre núcleos suaves. Como um bônus, obtemos positividade definida do núcleo resultante a partir da positividade definida do núcleo original. Por enquanto, os resultados se referem a núcleos suaves e não requerem suas representações em séries.

A princípio, precisamos de uma extensão da seguinte propriedade originalmente provada em [53]: se $U$ é um subconjunto aberto de $\mathbb{R}^{m+1}, K \in C^{r, r}(U \times U)$ e $(x, y) \in$ $U \times U$, então

$$
D^{\alpha, \beta} K(x, y)=\lim _{h \rightarrow 0} \frac{1}{h^{|\alpha+\beta|}} \prod_{i=1}^{m+1} \Delta_{x_{i}, h}^{\alpha_{i}} \prod_{j=1}^{m+1} \Delta_{y_{j}, h}^{\beta_{j}} K(x, y), \quad|\alpha|,|\beta| \leq r .
$$

Os operadores diferença aparecendo nesta fórmula são definidos por

$$
\begin{array}{r}
\Delta_{x_{i}, h} K(x, y):=K\left(x+h e_{i}, y\right)-K(x, y), \quad i=1, \ldots, m+1, \\
\Delta_{y_{j}, h} K(x, y):=K\left(x, y+h e_{j}\right)-K(x, y), \quad j=1, \ldots, m+1,
\end{array}
$$


e não têm relação com o operador de Laplace-Beltrami introduzido anteriormente. O incremento $h$ pode assumir qualquer valor, desde que o argumento permaneça no domínio $U$. Claramente, este é o caso quando $h$ está próximo de 0 . Para $h$ suficientemente pequeno, vê-se que os símbolos comutam, isto é, $\Delta_{x_{i}, h} \circ \Delta_{y_{j}, h}=\Delta_{y_{j}, h} \circ \Delta_{x_{i}, h}$. Estamos identificando $\Delta_{x_{i}, h}^{0}$ e $\Delta_{y_{j}, h}^{0}$ com o operador identidade e, se $r$ é um inteiro positivo, escrevendo $\Delta_{x_{i}, h}^{r}=\Delta_{x_{i}, h} \circ \cdots \circ \Delta_{x_{i}, h}(r$ vezes $)$.

A demonstração da fórmula limite anterior pode ser obtida tomando-se o limite na seguinte relação, obtida como uma aplicação do Teorema do Valor Médio usual:

$$
\prod_{i=1}^{m+1} \Delta_{x_{i}, h}^{\alpha_{i}} \prod_{j=1}^{m+1} \Delta_{y_{j}, h}^{\beta_{j}} K(x, y)=h^{|\alpha+\beta|} D^{\alpha, \beta} K\left(x+\Theta_{x}, y+\Theta_{y}\right)
$$

Aqui, $\alpha=\left(\alpha_{1}, \ldots, \alpha_{m+1}\right), \beta=\left(\beta_{1}, \ldots, \beta_{m+1}\right)$ e

$$
\Theta_{x}=\sum_{\alpha_{i} \neq 0} \sum_{\mu=1}^{\alpha_{i}} \theta_{\mu, i}^{x} e_{i}
$$

enquanto que $\theta_{\mu, i}^{x} \in[0, h], \mu=1, \ldots, \alpha_{i}$. Os números $\Theta_{x}$ e $\theta_{\mu, i}^{x}$ não dependem de $x$. Utilizamos os índices superiores e inferiores $x$ para chamar a atenção para a componente envolvida em cada passo e evitar a introdução de uma letra adicional na fórmula. Semelhante observação é aplicável quando o índice é $y$. A fórmula limite

$$
\lim _{h \rightarrow 0} D^{\alpha, \beta} K\left(x+\Theta_{x}, y+\Theta_{y}\right)=D^{\alpha, \beta} K(x, y)
$$

é uma consequência imediata. Se $r \leq m+1$, uma versão estendida de (3.1.4) toma a forma

$$
\begin{aligned}
\prod_{i_{r}=1}^{m+1} \Delta_{x_{i_{r}, h}}^{\alpha_{i_{r}}^{r}} \prod_{j_{r}=1}^{m+1} \Delta_{y_{j_{r}, h}}^{\beta_{j_{r}}^{r}} \cdots \prod_{i_{1}=1}^{m+1} \Delta_{x_{i_{1}}, h}^{\alpha_{i_{1}}^{1}} \prod_{j_{1}=1}^{m+1} \Delta_{y_{j_{1}}, h}^{\beta_{j_{1}}^{1}} K(x, y)=h^{\left|\alpha_{l}+\cdots+\alpha_{1}+\beta_{l}+\cdots+\beta_{1}\right|} \times \\
D^{\alpha_{r}, \beta_{r}} \ldots D^{\alpha_{1}, \beta_{1}} K\left(x+\Theta_{x}^{r}+\cdots+\Theta_{x}^{1}, y+\Theta_{y}^{r}+\cdots+\Theta_{y}^{1}\right),
\end{aligned}
$$

onde $\alpha_{l}=\left(\alpha_{l_{1}}^{l}, \ldots, \alpha_{l_{m+1}}^{l}\right), \beta_{l}=\left(\beta_{l_{1}}^{l}, \ldots, \beta_{l_{m+1}}^{l}\right), l=1, \ldots, r$ e $\Theta_{x}^{i}$ e $\Theta_{y}^{j}$ são definidos seguindo um procedimento semelhante ao de 3.1.5.

Isto garante a validade do seguinte resultado.

Teorema 3.1.1. Sejam $r$ um inteiro positivo e $K \in C^{s, s}(U \times U)$. Se $\alpha_{l}, \beta_{l}, l=1, \ldots, r$, são multi-índices satisfazendo $\sum_{l=1}^{r}\left|\alpha_{l}\right| \leq s$ e $\sum_{l=1}^{r}\left|\beta_{l}\right| \leq s$, então

$$
D^{\alpha_{r}, \beta_{r}} \ldots D^{\alpha_{1}, \beta_{1}} K=\lim _{h \rightarrow 0} \prod_{l=1}^{r} \frac{1}{h^{\left|\alpha_{l}+\beta_{l}\right|}} \prod_{i_{r}=1}^{m+1} \Delta_{x_{i_{r}}, h}^{\alpha_{i_{r}}^{r}} \prod_{j_{r}=1}^{m+1} \Delta_{y_{j_{r}}, h}^{\beta_{j_{r}}^{r}} \ldots \prod_{i_{1}=1}^{m+1} \Delta_{x_{i_{1}}, h}^{\alpha_{i_{1}}^{1}} \prod_{j_{1}=1}^{m+1} \Delta_{y_{j_{1}}, h}^{\beta_{j_{1}}^{1}} K
$$


A seguir, usamos a fórmula anterior para deduzir uma expressão em forma de limite para calcular o núcleo $K_{r}$ dado por

$$
K_{r}:=\sum_{p_{r}=1}^{m+1} \cdots \sum_{p_{1}=1}^{m+1} D_{x}^{2\left(e_{p_{r}}+\cdots+e_{p_{1}}\right)} \sum_{q_{r}=1}^{m+1} \cdots \sum_{q_{1}=1}^{m+1} D_{y}^{2\left(e_{q_{r}}+\cdots+e_{q_{1}}\right)} K .
$$

Teorema 3.1.2. Se $K \in C^{2 r, 2 r}(U \times U)$, então

$$
K_{r}=\lim _{h \rightarrow 0} \frac{1}{h^{4 r}} \sum_{p_{r}, q_{r}=1}^{m+1} \cdots \sum_{p_{1}, q_{1}=1}^{m+1} \Delta_{x_{p_{r}}, h}^{2} \Delta_{y_{q_{r}}, h}^{2} \cdots \Delta_{x_{p_{1}}, h}^{2} \Delta_{y_{q_{1}}, h}^{2} K .
$$

Demonstração: Aplicando (3.1.6), vemos que

$$
\begin{aligned}
K_{r}(x, y) & =\sum_{p_{r}, q_{r}=1}^{m+1} \cdots \sum_{p_{1}, q_{1}=1}^{m+1} D_{x}^{2\left(e_{p_{r}}+\cdots+e_{p_{1}}\right)} D_{y}^{2\left(e_{q_{r}}+\cdots+e_{q_{1}}\right)} K(x, y) \\
& =\sum_{p_{r}, q_{r}=1}^{m+1} \cdots \sum_{p_{1}, q_{1}=1}^{m+1} D^{2\left(e_{p_{r}}+\cdots+e_{p_{1}}\right), 2\left(e_{q_{r}}+\cdots+e_{q_{1}}\right)} K(x, y) \\
& =\sum_{p_{r}, q_{r}=1}^{m+1} \cdots \sum_{p_{1}, q_{1}=1}^{m+1} \lim _{h \rightarrow 0} D^{2\left(e_{p_{r}}+\cdots+e_{p_{1}}\right), 2\left(e_{q_{r}}+\cdots+e_{q_{1}}\right)} K\left(x+\Theta_{x}, y+\Theta_{y}\right) \\
& =\lim _{h \rightarrow 0} \sum_{p_{r}, q_{r}=1}^{m+1} \cdots \sum_{p_{1}, q_{1}=1}^{m+1} D^{2\left(e_{p_{r}}+\cdots+e_{p_{1}}\right), 2\left(e_{q_{r}}+\cdots+e_{q_{1}}\right)} K\left(x+\Theta_{x}, y+\Theta_{y}\right),
\end{aligned}
$$

para cada $x, y \in U$. Recorrendo a (3.1.4), obtemos

$$
\begin{aligned}
K_{r}(x, y) & =\lim _{h \rightarrow 0} \sum_{p_{r}, q_{r}=1}^{m+1} \cdots \sum_{p_{1}, q_{1}=1}^{m+1} \frac{1}{h^{4 r}} \prod_{i=1}^{m+1} \Delta_{x_{i}, h}^{\left[2\left(e_{p_{r}}+\cdots+e_{p_{1}}\right)\right]_{i}} \prod_{j=1}^{m+1} \Delta_{y_{j}, h}^{\left[2\left(e_{q_{r}}+\cdots+e_{q_{1}}\right)\right]_{j}} K(x, y) \\
& =\lim _{h \rightarrow 0} \frac{1}{h^{4 r}} \sum_{p_{r}, q_{r}=1}^{m+1} \cdots \sum_{p_{1}, q_{1}=1}^{m+1} \Delta_{x_{p_{r}}, h}^{2} \Delta_{y_{q_{r}}, h}^{2} \cdots \Delta_{x_{p_{1}}, h}^{2} \Delta_{y_{q_{1}}, h}^{2} K(x, y), \quad x, y \in U,
\end{aligned}
$$

que é a expressão que procurávamos.

Como

$$
\Delta_{y_{l}, h}^{2} K(x, y)=\sum_{\nu_{l}=0}^{2} d_{\nu_{l}} K\left(x, y+h \nu_{l} e_{l}\right), \quad x, y \in U
$$

onde

$$
d_{r}=(-1)^{r}\left(\begin{array}{l}
2 \\
r
\end{array}\right), \quad r=0,1,2
$$

vemos que

$$
\Delta_{x_{k}, h}^{2} \Delta_{y_{l}, h}^{2} K(x, y)=\sum_{\mu_{k}, \nu_{l}=0}^{2} d_{\mu_{k}} d_{\nu_{l}} K\left(x+h \mu_{k} e_{k}, y+h \nu_{l} e_{l}\right), \quad x, y \in U .
$$

Isto é o que precisamos para justificar o corolário a seguir. 
Corolário 3.1.3. A fórmula no Teorema 3.1.2 pode ser re-escrita como

$$
K_{r}(x, y)=\lim _{h \rightarrow 0} \frac{1}{h^{4 r}} \sum_{p_{r}, q_{r}=1}^{m+1} d_{\mu_{r}, \nu_{r}} \ldots \sum_{p_{1}, q_{1}=1}^{m+1} d_{\mu_{1}, \nu_{1}} K\left(x+h \sum_{k=1}^{r} \mu_{k} e_{p_{k}}, y+h \sum_{l=1}^{r} \nu_{l} e_{q_{l}}\right),
$$

para $x, y \in U$, onde

$$
d_{\mu_{j}, \nu_{j}}=\sum_{\mu_{j}, \nu_{j}=0}^{2} d_{\mu_{j}} d_{\nu_{j}}, \quad j=1, \ldots, r
$$

Antes de seguir em frente, relembramos o seguinte resultado técnico provado em [53].

Lema 3.1.4. Sejam $X$ e $Y$ conjuntos não vazios e $K$ um núcleo sobre $X \times X$. Tome $N+1$ funções $g_{\mu}: Y \rightarrow X, \mu=0, \ldots, N$, números complexos $\lambda_{0}, \ldots, \lambda_{N}$, e defina

$$
L(x, y):=\sum_{k, l=0}^{N} \lambda_{k} \overline{\lambda_{l}} K\left(g_{k}(x), g_{l}(y)\right), \quad x, y \in Y .
$$

Se $K$ é positivo definido sobre $X \times X$, então $L$ é positivo definido sobre $Y \times Y$.

Agora, retornamos ao caso esférico com a derivada de Laplace-Beltrami para apresentar o principal resultado desta seção.

Teorema 3.1.5. Seja r um inteiro positivo. Se $K \in C^{2 r, 2 r}\left(S^{m} \times S^{m}\right)$ é positivo definido, então $\mathcal{D}^{r, r} K$ é positivo definido.

Demonstração: Uma aplicação do Teorema 1.6.2 permite escrevermos

$$
\begin{aligned}
\mathcal{D}^{r, r} K & =m^{-2 r}\left(\Delta_{m}^{x} \Delta_{m}^{y}\right)^{r} K \\
& =m^{-2 r} \sum_{p_{1}=1}^{m+1} \cdots \sum_{p_{r}=1}^{m+1} D_{x}^{2\left(e_{p_{1}}+\cdots+e_{p_{r}}\right)} \sum_{q_{1}=1}^{m+1} \cdots \sum_{q_{r}=1}^{m+1} D_{y}^{2\left(e_{q_{1}}+\cdots+e_{q_{r}}\right)} K .
\end{aligned}
$$

Agora, relembrando como definimos a derivada usual sobre a esfera, vemos que a expressão na última linha acima define o núcleo $\widehat{K_{r}}$, que é um múltiplo não negativo de $K_{r}$ (o conjunto aberto $U$ é $\mathbb{R}_{*}^{m+1}$ ), com a característica particular de que $p_{k}=q_{k}$, $k=1, \ldots, r$. Neste caso, ao olharmos para a expressão de $K_{r}$ dada no Corolário 3.1.3. vemos que o núcleo aparecendo no limite coincide com a descrição dada no Lema 3.1.4. Portanto, aplicando o lema $r$ vezes concluímos que $\widehat{K_{r}}$ é não negativo definido, pois é limite pontual de uma sequência de núcleos positivos definidos. 


\subsection{Derivação termo a termo}

Supondo que o núcleo se encaixa na descrição dada no início do capítulo, analisamos agora sua diferenciabilidade termo a termo no sentido de Laplace-Beltrami. Estamos especialmente interessados na convergência da série resultante para a derivada do núcleo original. Resultados desta natureza têm importância técnica na análise de taxas de decaimento dos autovalores do operador integral gerado pelo núcleo. Antes de enunciar e provar cada resultado, enunciamos uma proposição contendo o resultado correspondente para derivadas usuais. Cada uma destas proposições pode ser encontrada demonstrada em [53].

Proposição 3.2.1. Sejam $\alpha$ um multi-índice não nulo e $K \in C^{|\alpha|,|\alpha|}\left(S^{m} \times S^{m}\right)$. Então, $\sum_{k=0}^{\infty} a_{k} D^{\mu} Y_{k}(x) D^{\nu} \overline{Y_{k}}(y)$ converge uniformemente para $D^{\mu, \nu} K(x, y)$ em $S^{m} \times S^{m}$, desde que $|\mu|,|\nu| \leq|\alpha|$.

Teorema 3.2.2. Sejam $\alpha$ um multi-índice não nulo e $K \in C^{|\alpha|,|\alpha|}\left(S^{m} \times S^{m}\right)$. Então, $\sum_{k=0}^{\infty} a_{k} \mathcal{D}^{s} Y_{k}(x) \mathcal{D}^{t} \overline{Y_{k}}(y)$ converge uniformemente para $\mathcal{D}^{s, t} K$ em $S^{m} \times S^{m}$, desde que $2 s, 2 t \leq|\alpha|$.

Demonstração: Suponhamos que $s$ e $t$ sejam tais que $2 s, 2 t \leq r$. Procedendo como na demonstração do Teorema 3.1.5, obtemos

$$
\mathcal{D}^{s, t} K=(-m)^{-(s+t)} \sum_{p_{1}=1}^{m+1} \cdots \sum_{p_{s}=1}^{m+1} \sum_{q_{1}=1}^{m+1} \cdots \sum_{q_{t}=1}^{m+1} D_{x}^{2\left(e_{p_{1}}+\cdots+e_{p_{s}}\right)} D_{y}^{2\left(e_{q_{1}}+\cdots+e_{q_{t}}\right)} K .
$$

Como $\left|e_{p_{1}}+\cdots+e_{p_{s}}\right|=s$ e $\left|e_{q_{1}}+\cdots+e_{q_{t}}\right|=t$, a Proposição 3.2.1 assegura que cada série

$$
\sum_{k=0}^{\infty} a_{k} D^{2\left(e_{p_{1}}+\cdots+e_{p_{s}}\right)} Y_{k} D^{2\left(e_{q_{1}}+\cdots+e_{q_{t}}\right)} \overline{Y_{k}}
$$

converge uniformemente para $D_{x}^{2\left(e_{p_{1}}+\cdots+e_{p_{s}}\right)} D_{x}^{2\left(e_{q_{1}}+\cdots+e_{q_{t}}\right)} K$ em $S^{m} \times S^{m}$. Consequentemente,

$$
\sum_{k=0}^{\infty} a_{k} \sum_{p_{1}=1}^{m+1} \cdots \sum_{p_{s}=1}^{m+1} \sum_{q_{1}=1}^{m+1} \cdots \sum_{q_{t}=1}^{m+1} D^{2\left(e_{p_{1}}+\cdots+e_{p_{s}}\right)} Y_{k} D^{2\left(e_{q_{1}}+\cdots+e_{q_{t}}\right)} \overline{Y_{k}}
$$

converge uniformemente para $D_{x}^{2\left(e_{p_{1}}+\cdots+e_{p_{s}}\right)} D_{x}^{2\left(e_{q_{1}}+\cdots+e_{q_{t}}\right)} K$ em $S^{m} \times S^{m}$, isto é, a série $\sum_{k=0}^{\infty} a_{k} \mathcal{D}^{s} Y_{k}(x) \mathcal{D}^{t} \overline{Y_{k}}(y)$ converge uniformemente para $\mathcal{D}^{s, t} K$ em $S^{m} \times S^{m}$.

O corolário a seguir é um análogo do Teorema 3.1.5 com uma pequena alteração nas hipóteses.

Corolário 3.2.3. Sejam $\alpha$ um multi-índice não nulo e $K \in C^{|\alpha|,|\alpha|}\left(S^{m} \times S^{m}\right)$. Se $2 s \leq|\alpha|$ e $K$ é positivo definido, então $\mathcal{D}^{s, s} K$ é positivo definido. 
A seguir, pretendemos analisar a recíproca do Teorema 3.2.2.

Proposição 3.2.4. Seja $\alpha$ um multi-índice não nulo para o qual $Y_{k} \in C^{|\alpha|}\left(S^{m}\right), k=$ $0,1, \ldots$ Sejam $\mu$ e $\nu$ multi-indices tais que $|\mu|,|\nu| \leq|\alpha|$. Se $\sum_{k=0}^{\infty} a_{k} D^{\mu} Y_{k}(x) D^{\nu} \overline{Y_{k}}(y)$ converge uniformemente em $S^{m} \times S^{m}$, então $D^{\mu, \nu} K(x, y)$ existe, é contínuo e coincide com a série.

Teorema 3.2.5. Suponhamos que cada $Y_{k}$ pertence a $C^{2 r}\left(S^{m}\right)$, para algum inteiro não negativo $r$. Sejam s e $t$ inteiros tais que $2 s, 2 t<r$. Se $\sum_{k=0}^{\infty} a_{k} D^{2 \alpha} Y_{k}(x) D^{2 \beta} \overline{Y_{k}}(y)$ converge uniformemente quando $|\alpha|<s$ e $|\beta|<t$, então $\mathcal{D}^{s, t} K(x, y)$ existe, é contínuo $e$

$$
\mathcal{D}^{s, t} K(x, y)=\sum_{k=0}^{\infty} a_{k} \mathcal{D}^{s} Y_{k}(x) \mathcal{D}^{t} \overline{Y_{k}}(y)
$$

Demonstração: Seja $S=\sum_{k=0}^{\infty} a_{k} \mathcal{D}^{s} Y_{k}(x) \mathcal{D}^{t} \overline{Y_{k}}(y)$. Como cada $Y_{k}$ é suficientemente suave, podemos escrever

$$
\begin{aligned}
S & :=(-m)^{-(s+t)} \sum_{k=0}^{\infty} a_{k}\left(\Delta_{m}\right)^{s} Y_{k}(x)\left(\Delta_{m}\right)^{t} \overline{Y_{k}}(y) \\
& =(-m)^{-(s+t)} \sum_{k=0}^{\infty} a_{k} \sum_{p_{1}=1}^{m+1} \cdots \sum_{p_{s}=1}^{m+1} D^{2\left(e_{p_{1}}+\cdots+e_{p_{s}}\right)} Y_{k}(x) \sum_{q_{1}=1}^{m+1} \cdots \sum_{q_{t}=1}^{m+1} D^{2\left(e_{q_{1}}+\cdots+e_{q_{t}}\right)} \overline{Y_{k}}(y) .
\end{aligned}
$$

Se $\sum_{k=0}^{\infty} a_{k} D^{2 \alpha} Y_{k}(x) D^{2 \beta} \overline{Y_{k}(y)}$ converge uniformemente quando $|\alpha| \leq s$ and $|\beta| \leq t$, podemos dar mais um passo e obter

$$
S=(-m)^{-(s+t)} \sum_{p_{1}=1}^{m+1} \cdots \sum_{p_{s}=1}^{m+1} \sum_{q_{1}=1}^{m+1} \cdots \sum_{q_{t}=1}^{m+1} \sum_{k=0}^{\infty} a_{k} D^{2\left(e_{p_{1}}+\cdots+e_{p_{s}}\right)} Y_{k}(x) D^{2\left(e_{q_{1}}+\cdots+e_{q_{t}}\right)} \bar{Y}_{k}(y) .
$$

Finalmente, uma aplicação da Proposição 3.2.4 nos leva a

$$
S=(-m)^{-(s+t)} \sum_{p_{1}=1}^{m+1} \cdots \sum_{p_{s}=1}^{m+1} \sum_{q_{1}=1}^{m+1} \cdots \sum_{q_{t}=1}^{m+1} D^{2\left(e_{p_{1}}+\cdots+e_{p_{s}}\right)} D^{2\left(e_{q_{1}}+\cdots+e_{q_{t}}\right)} K(x, y) .
$$

Como o lado direito da última identidade coincide com

$$
(-m)^{-(s+t)}\left(\Delta_{m}^{x}\right)^{s}\left(\Delta_{m}^{y}\right)^{t} K(x, y)=\mathcal{D}^{s, t} K(x, y)
$$

a demonstração está completa.

As hipóteses sobre $K$ nos Teoremas 3.2.2 e 3.2.5, e no Corolário 3.2.3, não envolvem a derivada de Laplace-Beltrami do núcleo. Preferimos manter os resultados desta forma porque assim os enunciados ficam mais simples. Para encerrar a seção, vamos explicar 
como trocar as hipóteses de derivação usual por outras envolvendo derivação no sentido de Laplace-Beltrami. Para isto, definimos o espaço do tipo Sobolev (veja [51, p. 37]).

$$
W_{2, m}^{2 r}:=\left\{f \in X:\left(-\Delta_{m}\right)^{r}(f) \in L^{2}\left(S^{m}\right)\right\} .
$$

O espaço $W_{2}^{r}$ pode ser alternativamente caracterizado como o espaço de todas as funções complexas $f$ sobre $S^{m}$ para as quais existe $g \in L^{2}\left(S^{m}\right)$ tal que

$$
\mathcal{Y}_{n}(g)=\left(\frac{n(n+m-1)}{m}\right)^{r} \mathcal{Y}_{n}(f), \quad n=0,1, \ldots
$$

Relembrando (1.6.7), vemos que se $f \in W_{2}^{r}$ e $g$ é a correspondente função fornecida pela caracterização recém descrita, então $\left(-\Delta_{m}\right)^{r}(f)=(m)^{r} g$. Em outras palavras, provamos a validade da seguinte inclusão: $W_{2}^{r} \subset W_{2, m}^{2 r}$. Finalmente, usando o clássico Teorema da Imersão de Sobolev ([58]), vemos que se $r \geq s+m / 2$, então $W_{2, m}^{r}$ pode ser continuamente imerso em $C^{s}\left(S^{m}\right)$. Assim, concluímos que $W_{2}^{r}$ pode ser continuamente imerso em $C^{s}\left(S^{m}\right)$, desde que $2 r \geq s+m / 2$. Desta forma, por exemplo, uma hipótese razoável sobre $K$ no Teorema 3.2 .2 seria $\mathcal{D}_{x}^{\gamma} K, \mathcal{D}_{y}^{\gamma} K \in L^{2}\left(S^{m}\right)$, sempre que $2|\gamma| \geq$ $|\alpha|+m / 2$. 



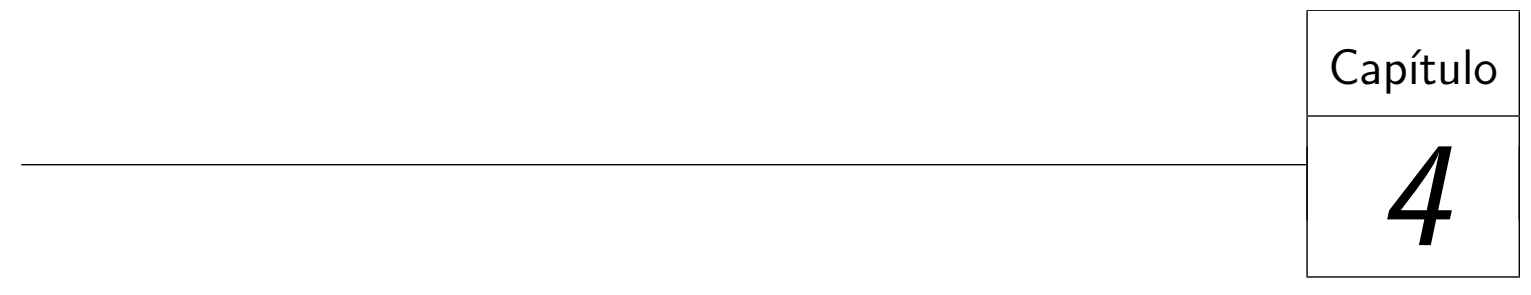

\section{Decaimento de autovalores}

Neste capítulo apresentamos os principais resultados da tese, onde obtemos taxas de decaimento para valores singulares e autovalores de operadores integrais gerados por núcleos com quadrado integrável sobre a esfera unitária em $\mathbb{R}^{m+1}$, para $m \geq 2$, utilizando hipóteses sobre certas derivadas do núcleo gerador ou sobre o operador integral gerado por tais derivadas. Este tipo de problema é comum na literatura, mas as hipóteses básicas de suavidade geralmente são definidas usando-se condições de diferenciabilidade usual em $\mathbb{R}^{m+1}$. No que diz respeito aos resultados seguintes, estas hipóteses básicas são definidas via diferenciabilidade de Laplace-Beltrami, um conceito genuinamente esférico. Fica claro nos resultados que as taxas alcançadas dependem da ordem de diferenciabilidade usada para definir as condições de suavidade e da dimensão $m$ da esfera. Também provamos que, em determinando sentido, tais taxas de decaimento são ótimas.

\subsection{Resultados principais}

Começamos introduzindo algumas notações para facilitar o entendimento dos resultados e o trabalho nas demonstrações. Para $r \in \mathbb{Z}_{+}$, achamos conveniente escrever

$$
K_{0, r}(x, y):=\mathcal{D}_{y}^{r} K(x, y), \quad x, y \in S^{m}
$$

e abandonar a notação de derivada. Seguindo esta notação, o operador integral gerado pelo núcleo $K_{0, r}$ será denotado por $\mathcal{K}_{0, r}$. Neste ponto, também torna-se conveniente 
introduzir a seguinte notação: um núcleo $K \in L^{2}\left(S^{m} \times S^{m}\right)$ pertence ao espaço $W_{2}^{r}$, se $K(x, \cdot) \in W_{2}^{r}, x \in S^{m}$ q.s..

Para seguir em frente, precisamos ordenar os autovalores do operador $J^{r}$ de acordo com o Teorema de Hilbert-Schmidt (Teorema 1.3.14). Em outras palavras, relembrando (1.7.1), supomos que eles estão ordenados em ordem decrescente contando as repetições impostas pelas fórmulas $J^{r} 1=1 \mathrm{e}$

$$
J^{r} Y=\frac{m^{r}}{n^{r}(n+m-1)^{r}} Y, \quad Y \in \mathcal{H}_{n}^{m+1}, \quad n=1,2, \ldots
$$

Assim, de acordo com 1.4.5), vemos que a sequência $\left\{\lambda_{n}\left(J^{r}\right)\right\}$ dos autovalores de $J^{r}$ é ordenada em blocos de tal forma que o primeiro bloco contém o autovalor 1 e o $(n+1)$-ésimo bloco $(n \geq 1)$ contém $N(m, n)$ entradas iguais a $m^{r} n^{-r}(n+m-1)^{-r}$. Para futuras referências, relembramos 1.4.7) e observamos que a primeira entrada do $(n+1)$-ésimo bloco corresponde ao índice

$$
N(m, 0)+N(m, 1)+\cdots+N(m, n-1)+1=N(m+1, n-1)+1, \quad n \geq 1 .
$$

Analogamente, a última entrada corresponde ao índice

$$
N(m, 0)+N(m, 1)+\cdots+N(m, n-1)+N(m, n)=N(m+1, n), \quad n \geq 1 .
$$

O próximo lema é ponto chave nas demonstrações dos resultados principais deste capítulo. Supondo $K$ suficientemente suave, ele auxilia na obtenção de uma estimativa para os valores singulares de $\mathcal{K}$ em função dos autovalores do operador integral gerado por uma derivada de $K$ e dos autovalores do operador de integral de Laplace-Beltrami.

Lema 4.1.1. Seja $K$ um elemento de $W_{2}^{r}$. Se $\mathcal{K}_{0, r}$ é limitado, então

$$
s_{n+1}(\mathcal{K}) \leq s_{n}\left(\mathcal{K}_{0, r} J^{r}\right), \quad n=1,2, \ldots
$$

Demonstração: Consideremos a projeção ortogonal $Q$ de $L^{2}\left(S^{m}\right)$ sobre $\oplus_{\ell=1}^{\infty} \mathcal{H}_{\ell}^{m+1}$. Como $I-Q$ é uma projeção sobre o complemento ortogonal de $\oplus_{\ell=1}^{\infty} \mathcal{H}_{\ell}^{m+1}$, então $\mathcal{K}-\mathcal{K} Q$ é um operador sobre $L^{2}\left(S^{m}\right)$ de posto no máximo 1. Usando o item (ii) do Teorema 1.3.17, deduzimos que

$$
s_{n+1}(\mathcal{K}) \leq s_{n}(\mathcal{K}-\mathcal{K}(I-Q))=s_{n}(\mathcal{K} Q), \quad n=1,2, \ldots
$$

Para prosseguir, precisamos de uma decomposição conveniente para o operador $\mathcal{K} Q$. Analisando sua ação sobre um elemento genérico $f$ de $L^{2}\left(S^{m}\right)$ e usando 1.7 .2 , vemos que

$$
\mathcal{K} Q(f)=\int_{S^{m}} K(\cdot, y) Q f(y) d \sigma_{m}(y)=\int_{S^{m}} K(\cdot, y) \mathcal{D}^{r} J^{r} Q f(y) d \sigma_{m}(y)
$$


Como $K \in W_{2}^{r}$, empregamos 1.6.5 para obter

$$
\mathcal{K} Q(f)=\int_{S^{m}} K_{0, r}(\cdot, y) J^{r}(Q f)(y) d \sigma_{m}(y)=\mathcal{K}_{0, r} J^{r} Q(f),
$$

isto é, $\mathcal{K} Q=\mathcal{K}_{0, r} J^{r} Q$. Agora, supondo que $\mathcal{K}_{0, r}$ é limitado, podemos aplicar 4.1.5 e o item (i) do Teorema 1.3 .17 para garantir que

$$
s_{n+1}(\mathcal{K}) \leq s_{n}(\mathcal{K} Q) \leq\|Q\| s_{n}\left(\mathcal{K}_{0, r} J^{r}\right) \leq s_{n}\left(\mathcal{K}_{0, r} J^{r}\right), \quad n=1,2, \ldots
$$

A demonstração está completa.

A seguir, destacamos algumas desigualdades técnicas a serem usadas nas demonstrações seguintes. A primeira é um refinamento da ordem de convergência $N(m, n)=$ $O\left(n^{m-1}\right)$.

Lema 4.1.2. Existe um inteiro $\beta(m) \geq 1$ tal que

$$
N(m+1, n) \leq 2 n^{m}, \quad n \geq \beta(m) .
$$

Demonstração: Segue da equação 1.4.6 que

$$
\lim _{n \rightarrow \infty} \frac{N(m+1, n)}{n^{m}}=\frac{2}{m !} \leq 1, \quad m \geq 2 .
$$

E isto é suficiente para garantir a veracidade do resultado .

Lema 4.1.3. Se $m$ é um inteiro maior ou igual a 2, então existe um inteiro $\gamma(m) \geq 1$ tal que

$$
(n+1)^{m}-\left(n^{m}+1\right)+1 \leq n^{m}, \quad n \geq \gamma(m) .
$$

Demonstração: Isto segue da equação

$$
\frac{(n+1)^{m}-\left(n^{m}+1\right)+1}{n^{m}}=\sum_{j=0}^{m-1}\left(\begin{array}{c}
m \\
j
\end{array}\right) n^{j-m},
$$

após observarmos que a soma do lado direito se aproxima de 0 , quando $n \rightarrow \infty$.

Lema 4.1.4. Sejam $m$ um inteiro maior ou igual a 2, $r$ um inteiro não negativo $e$ $\epsilon \in(0,1)$. Então, existe $\delta=\delta(m, \epsilon) \geq 1$ tal que

$$
(1-\epsilon) n^{2 r} \leq(n-1)^{r}(n+m-2)^{r}, \quad n \geq \delta .
$$

Demonstração: Nos casos $m=2,3$, é suficiente observar que $(n-1)^{r}(n+m-2)^{r} n^{-2 r}$ se aproxima de 1 pela esquerda, quando $n \rightarrow \infty$. Para o caso $m>3$, a sequência $(n-1)^{r}(n+m-2)^{r} n^{-2 r}$ se aproxima de 1 pela direita, quando $n \rightarrow \infty$. Logo, a propriedade segue da desigualdade $1-\epsilon<1$. 
Lema 4.1.5. Seja $m$ um inteiro maior ou igual a 2. Então, existe uma constante $c=c(m)>0$ tal que

$$
n^{m-1} \leq c N(m, n), \quad n=1,2, \ldots
$$

Demonstração: Lembrando (1.4.6), vemos que

$$
\lim _{n \rightarrow \infty} \frac{n^{m-1}}{N(m, n)}=\frac{(m-1) !}{2} .
$$

De acordo com a Definição 1.1.8, temos que $n^{m-1}=O(N(m, n))$. Portando, o resultado segue da Proposição 1.1.9.

Estamos prontos para atacar os principais problemas propostos para esta tese. É importante enfatizar que os resultados a seguir levam em consideração a ordenação dos autovalores e dos valores singulares da forma como mencionamos anteriormente. Primeiro, provamos um teorema sem a hipótese de $L^{2}$-positividade definida sobre $K$ e obtemos uma taxa de decaimento para a sequência dos valores singulares de $\mathcal{K}$.

Teorema 4.1.6. Sejam $r$ um inteiro positivo maior ou igual a $(m+2) / 2, K$ um elemento de $W_{2}^{r}$ e $p \in(m+1,2 r]$. Se $\mathcal{K}_{0, r}$ é limitado, então

$$
s_{n}(\mathcal{K})=o\left(n^{-1-(2 r-p) / m}\right) .
$$

Demonstração: Começamos supondo que $\mathcal{K}_{0, r}$ é limitado. Nosso objetivo é provar que

$$
\sum_{n=1}^{\infty} n^{(2 r-p) / m} s_{n}(\mathcal{K})<\infty,
$$

pois assim o Teorema 1.1.11 garante o resultado. Na primeira metade da demonstração pretendemos provar a convergência da série

$$
\sum_{n=1}^{\infty} n^{2 r+m-p} s_{n^{m}}(\mathcal{K}) .
$$

Uma aplicação do Teorema 1.3.17.(i) na desigualdade do Lema 4.1.1 mostra que

$$
s_{n+1}(\mathcal{K}) \leq\left\|\mathcal{K}_{0, r}\right\| s_{n}\left(J^{r}\right), \quad n=1,2, \ldots
$$

Como $J^{r}$ é autoadjunto e seus autovalores são positivos, o Teorema 1.3 .18 implica que

$$
s_{n+1}(\mathcal{K}) \leq \lambda_{n}\left(J^{r}\right)\left\|\mathcal{K}_{0, r}\right\|, \quad n=1,2, \ldots
$$

Mantendo em mente as equações 4.1.3 e 4.1.4 e lembrando da forma em blocos como os autovalores de $J^{r}$ estão dispostos, podemos escrever

$$
\begin{aligned}
\sum_{k=N(m+1, n-2)+1}^{N(m+1, n-1)} s_{k+1}(\mathcal{K}) & \leq \frac{m^{r}}{(n-1)^{r}(n+m-2)^{r}} \sum_{k=N(m+1, n-2)+1}^{N(m+1, n-1)}\left\|\mathcal{K}_{0, r}\right\| \\
& =\frac{m^{r}}{(n-1)^{r}(n+m-2)^{r}}\left\|\mathcal{K}_{0, r}\right\| N(m, n-1),
\end{aligned}
$$


para $n=2,3, \ldots$ Logo,

$$
(n-1)^{r}(n+m-2)^{r} \sum_{k=N(m+1, n-2)+1}^{N(m+1, n-1)} s_{k+1}(\mathcal{K}) \leq m^{r}\left\|\mathcal{K}_{0, r}\right\| N(m, n-1), \quad n=2,3, \ldots
$$

e usando o fato de a sequência $\left\{s_{n}(\mathcal{K})\right\}$ ser decrescente, podemos estimar na desigualdade anterior para obter

$$
(n-1)^{r}(n+m-2)^{r} s_{N(m+1, n-1)+1}(\mathcal{K}) \leq m^{r}\left\|\mathcal{K}_{0, r}\right\|, \quad n=2,3, \ldots
$$

Invocando o Lema 4.1.2, podemos selecionar $\beta(m) \geq 1$ tal que

$$
N(m+1, n-1)+1 \leq 2(n-1)^{m}+1 \leq(2 n)^{m}, \quad n \geq \beta(m),
$$

e reduzir a desigualdade (4.1.8) a

$$
(n-1)^{r}(n+m-2)^{r} s_{(2 n)^{m}}(\mathcal{K}) \leq m^{r}\left\|\mathcal{K}_{0, r}\right\|, \quad n \geq \beta(m) .
$$

Este é o ponto onde escolhemos $\epsilon \in(0,1)$ e tomamos $\delta$ da forma descrita no Lema 4.1.4 para escrever

$$
(1-\epsilon) n^{2 r} s_{(2 n)^{m}}(\mathcal{K}) \leq m^{r}\left\|\mathcal{K}_{0, r}\right\|, \quad n \geq \max \{\delta, \beta(m)\}
$$

Então, fica claro que

$$
\sum_{n \geq \max \{\delta, \beta(m)\}} n^{2 r+m-p} s_{(2 n)^{m}}(\mathcal{K}) \leq \frac{m^{r}}{1-\epsilon}\left\|\mathcal{K}_{0, r}\right\| \sum_{n \geq \max \{\delta, \beta(m)\}} n^{m-p}<\infty,
$$

pois $p-m>1$. Consequentemente,

$$
\sum_{n \geq \max \{\delta, \beta(m)\}}(2 n)^{2 r+m-p} s_{(2 n)^{m}}(\mathcal{K}) \leq 2^{2 r+m} \sum_{n \geq \max \{\delta, \beta(m)\}} n^{2 r+m-p} s_{(2 n)^{m}}(\mathcal{K})<\infty
$$

e

$$
\sum_{n \geq \max \{\delta, \beta(m)\}}(2 n+1)^{2 r+m-p} s_{(2 n+1)^{m}}(\mathcal{K}) \leq 4^{2 r+m} \sum_{n \geq \max \{\delta, \beta(m)\}} n^{2 r+m-p} s_{(2 n)^{m}}(\mathcal{K})<\infty .
$$

A convergência da série em 4.1.7) segue, visto que

$$
\sum_{n=2}^{\infty} n^{2 r+m-p} s_{n^{m}}(\mathcal{K})=\sum_{n=1}^{\infty}\left[(2 n)^{2 r+m-p} s_{(2 n)^{m}}(\mathcal{K})+(2 n+1)^{2 r+m-p} s_{(2 n+1)^{m}}(\mathcal{K})\right]<\infty
$$

Para encerrar a demonstração, usamos esta convergência para mostrar que

$$
\sum_{n=l^{m+1}}^{\infty} n^{(2 r-p) / m} s_{n}(\mathcal{K})<\infty,
$$


quando $l$ é suficientemente grande. Para fazer isto, mostramos que a seguinte reordenação de 4.1.9

$$
\sum_{n=l}^{\infty} \sum_{k=0}^{(n+1)^{m}-\left(n^{m}+1\right)}\left(n^{m}+k\right)^{(2 r-p) / m} s_{n^{m}+k}(\mathcal{K})
$$

converge quando $l$ é grande o suficiente. Chamando a soma interna em 4.1.10 de $S(n)$, segue do Lema 4.1 .3 que

$$
S(n) \leq\left[(2 n)^{m}\right]^{(2 r-p) / m} \sum_{k=0}^{(n+1)^{m}-\left(n^{m}+1\right)} s_{n^{m}+k}(\mathcal{K}), \quad n \geq \gamma(m) .
$$

Como a sequência $\left\{s_{n}(\mathcal{K})\right\}$ é decrescente, segue de 4.1.11) que

$$
S(n) \leq(2 n)^{2 r-p} s_{n^{m}}(\mathcal{K})\left[(n+1)^{m}-\left(n^{m}+1\right)+1\right], \quad n \geq \gamma(m) .
$$

Recorrendo novamente ao Lema 4.1 .3 , vemos que

$$
S(n) \leq 4^{r} n^{2 r+m-p} s_{n^{m}}(\mathcal{K}), \quad n \geq \gamma(m)
$$

Portanto, se $l \geq \max \{\delta, \beta(m), \gamma(m)\}$, evocamos 4.1.7 e concluímos que

$$
\sum_{n=l}^{\infty} S(n) \leq 4^{r} \sum_{n=l}^{\infty} n^{2 r+m-p} s_{n^{m}}(\mathcal{K})<\infty
$$

Uma aplicação do Teorema 1.1.11 encerra a demonstração.

Antes de seguir em frente, observamos que o fato de existirem as derivadas $\mathcal{D}_{y}^{r} K(x, \cdot)$ para quase todo $x \in S^{m}$ não implica que $\mathcal{K}_{0, r}$ é limitado. Desta forma, vemos que é razoável a hipótese sobre $\mathcal{K}_{0, r}$ no teorema anterior. Além disso, é fácil ver que quanto menor o parâmetro $p$, melhor a estimativa.

Nos próximos dois resultados incorporamos a $L^{2}$-positividade definida como hipótese. Assim, eles descreverão taxas de decaimento para os autovalores de $\mathcal{K}$ sob certas hipóteses que impomos para $K_{0, r}$ e $\mathcal{K}_{0, r}$, respectivamente.

Teorema 4.1.7. Sejam $K$ um núcleo $L^{2}$-positivo definido em $W_{2}^{r}$. Se $K_{0, r}$ pertence a $L^{2}\left(S^{m} \times S^{m}\right)$, então

$$
\lambda_{n}(\mathcal{K})=o\left(n^{-1 / 2-(4 r-1) / 2 m}\right)
$$

Demonstração: Procedemos como na demonstração do Teorema 4.1.6. Começamos supondo que $K_{0, r} \in L^{2}\left(S^{m} \times S^{m}\right)$ com o objetivo de provar que

$$
\sum_{n=1}^{\infty} n^{(4 r-1) / m} \lambda_{n}^{2}(\mathcal{K})<\infty
$$


Combinando o Lema 4.1.1 com o item (iii) do Teorema 1.3.17, deduzimos as desigualdades

$$
s_{n+k}(\mathcal{K}) \leq s_{n+k-1}\left(\mathcal{K}_{0, r} J^{r}\right) \leq s_{k}\left(\mathcal{K}_{0, r}\right) s_{n}\left(J^{r}\right), \quad n, k=1,2, \ldots
$$

Além disso, os operadores $\mathcal{K}$ e $J^{r}$ são autoadjuntos e positivos, o que permite que apliquemos o Teorema 1.3 .18 para escrever

$$
\lambda_{n+k}(\mathcal{K}) \leq s_{k}\left(\mathcal{K}_{0, r}\right) \lambda_{n}\left(J^{r}\right), \quad n, k=1,2, \ldots
$$

Nada impede que, por conveniência, re-escrevamos 4.1 .13 na forma

$$
\lambda_{N(m+1, n)+k}(\mathcal{K}) \leq s_{k}\left(\mathcal{K}_{0, r}\right) \lambda_{N(m+1, n)}\left(J^{r}\right), \quad n, k=1,2, \ldots
$$

A seguir, quadramos ambos os lados da desigualdade (4.1.14 e somamos em $k$, deixando o contador correr dentro do $(n+1)$-ésimo bloco da sequência de autovalores de $J^{r}$. Isto quer dizer que para todo inteiro $n$ maior ou igual a 1 ,

$$
\begin{aligned}
\sum_{k=N(m+1, n-1)+1}^{N(m+1, n)} \lambda_{N(m+1, n)+k}^{2}(\mathcal{K}) & \leq \lambda_{N(m+1, n)}^{2}\left(J^{r}\right) \sum_{k=N(m+1, n-1)+1}^{N(m+1, n)} s_{k}^{2}\left(\mathcal{K}_{0, r}\right) \\
& =\frac{m^{2 r}}{n^{2 r}(n+m-1)^{2 r}} \sum_{k=N(m+1, n-1)+1}^{N(m+1, n)} s_{k}^{2}\left(\mathcal{K}_{0, r}\right) .
\end{aligned}
$$

Resumindo, para todo inteiro $n$ maior ou igual a 1 , temos

$$
n^{2 r}(n+m-1)^{2 r} \sum_{k=N(m+1, n-1)+1}^{N(m+1, n)} \lambda_{N(m+1, n)+k}^{2}(\mathcal{K}) \leq m^{2 r} \sum_{k=N(m+1, n-1)+1}^{N(m+1, n)} s_{k}^{2}\left(\mathcal{K}_{0, r}\right) .
$$

Estimando o lado esquerdo da última desigualdade obtida, vemos que

$$
n^{4 r} \sum_{k=N(m+1, n-1)+1}^{N(m+1, n)} \lambda_{N(m+1, n)+k}^{2}(\mathcal{K}) \leq m^{2 r} \sum_{k=N(m+1, n-1)+1}^{N(m+1, n)} s_{k}^{2}\left(\mathcal{K}_{0, r}\right), \quad n=1,2, \ldots
$$

Somando em $n$ e lembrando do Teorema 1.3.23, obtemos

$$
\sum_{n=1}^{\infty} n^{4 r} \sum_{k=N(m+1, n-1)+1}^{N(m+1, n)} \lambda_{N(m+1, n)+k}^{2}(\mathcal{K}) \leq m^{2 r}\left\|K_{0, r}\right\|_{2}^{2}<\infty
$$

Para proceder, aplicamos o Lema 4.1 .2 para selecionar um inteiro $\beta(m) \geq 1$ tal que

$$
2 N(m+1, n) \leq 2^{2} n^{m} \leq(2 n)^{m}, \quad n \geq \beta(m)
$$


O Lema 4.1.5 e o decrescimento de $\left\{\lambda_{n}(\mathcal{K})\right\}$ implicam

$$
\begin{aligned}
\sum_{n=\beta(m)}^{\infty}(2 n)^{4 r+m-1} \lambda_{(2 n)^{m}}^{2}(\mathcal{K}) & \leq C \sum_{n=\beta(m)}^{\infty}(2 n)^{4 r} N(m, n) \lambda_{(2 n)^{m}}^{2}(\mathcal{K}) \\
& \leq C 2^{4 r} \sum_{n=1}^{\infty} n^{4 r} \sum_{k=N(m+1, n-1)+1}^{N(m+1, n)} \lambda_{N(m+1, n)+k}^{2}(\mathcal{K}),
\end{aligned}
$$

onde $C$ é uma constante dependendo somente de $m$. Logo, segue de 4.1.15 que

$$
\sum_{n=\beta(m)}^{\infty}(2 n)^{4 r+m-1} \lambda_{(2 n)^{m}}^{2}(\mathcal{K})<\infty .
$$

Além disso, 4.1.16 garante que

$$
\begin{aligned}
\sum_{n=\beta(m)}^{\infty}(2 n+1)^{4 r+m-1} \lambda_{(2 n+1)^{m}}^{2}(\mathcal{K}) & \leq 2^{4 r+m} \sum_{n=\beta(m)}^{\infty}(2 n)^{4 r+m-1} \lambda_{(2 n+1)^{m}}^{2}(\mathcal{K}) \\
& \leq 2^{4 r+m} \sum_{n=\beta(m)}^{\infty}(2 n)^{4 r+m-1} \lambda_{(2 n)^{m}}^{2}(\mathcal{K})<\infty .
\end{aligned}
$$

Somando as duas últimas séries, vemos que

$$
\sum_{n=\beta(m)}^{\infty} n^{4 r+m-1} \lambda_{n^{m}}^{2}(\mathcal{K})<\infty
$$

Agora, observamos que

$$
\sum_{n=1}^{\infty} n^{(4 r-1) / m} \lambda_{n}^{2}(\mathcal{K})=\sum_{n=1}^{\infty} \sum_{k=0}^{(n+1)^{m}-\left(n^{m}+1\right)}\left(n^{m}+k\right)^{(4 r-1) / m} \lambda_{n^{m}+k}^{2}(\mathcal{K})
$$

e mostramos que a série dupla acima é convergente. De fato, evocando o Lema 4.1.3 e o decrescimento de $\left\{\lambda_{n}(\mathcal{K})\right\}$, segue de 4.1.17) que

$$
\begin{aligned}
\sum_{n=l}^{\infty} \sum_{k=0}^{(n+1)^{m}-\left(n^{m}+1\right)}\left(n^{m}+k\right)^{(4 r-1) / m} \lambda_{n^{m}+k}^{2}(\mathcal{K}) & \leq \sum_{n=l}^{\infty} \sum_{k=0}^{(n+1)^{m}-\left(n^{m}+1\right)}\left(2 n^{m}\right)^{(4 r-1) / m} \lambda_{n^{m}}^{2}(\mathcal{K}) \\
& \leq 2^{4 r / m} \sum_{n=l}^{\infty} n^{4 r+m-1} \lambda_{n^{m}}^{2}(\mathcal{K}) \\
& <\infty,
\end{aligned}
$$

contanto que $l \geq \max \{\gamma(m), \beta(m)\}$. Isto é suficiente para garantir a convergência da série em 4.1.12 e que uma aplicação do Teorema 1.1.11 encerra a demonstração. 
Ao trocarmos a hipótese básica de $K_{0, r}$ no Teorema 4.1.7 pela nuclearidade de $\mathcal{K}_{0, r}$, obtemos a seguinte melhoria na taxa de decaimento encontrada.

Teorema 4.1.8. Sejam $K$ um núcleo $L^{2}$-positivo definido em $W_{2}^{r}$. Se $\mathcal{K}_{0, r}$ é nuclear, então

$$
\lambda_{n}(\mathcal{K})=o\left(n^{-1-(2 r-1) / m}\right)
$$

Demonstração: Supomos que $\mathcal{K}_{0, r}$ é nuclear e provamos que

$$
\sum_{n=1}^{\infty} n^{(2 r-1) / m} \lambda_{n}(\mathcal{K})<\infty
$$

Seguimos a idéia da demonstração do Teorema 4.1.7 até (4.1.13). A partir deste ponto, podemos escrever

$$
n^{2 r} \lambda_{N(m+1, n)+k}(\mathcal{K}) \leq n^{r}(n+m-1)^{r} \lambda_{N(m+1, n)+k}(\mathcal{K}) \leq m^{r} s_{k}\left(\mathcal{K}_{0, r}\right) \quad n, k=1,2 \ldots
$$

Somando em $k$ e $n$ da mesma forma que fizemos no teorema anterior, encontramos

$$
\sum_{n=2}^{\infty} n^{2 r} \sum_{k=N(m+1, n-1)+1}^{N(m+1, n)} \lambda_{N(m+1, n)+k}(\mathcal{K}) \leq m^{r} \sum_{n=2}^{\infty} \sum_{k=N(m+1, n-1)+1}^{N(m+1, n)} s_{k}\left(\mathcal{K}_{0, r}\right) .
$$

Evocando 1.3.3, vemos que a soma dupla do lado direito de 4.1.20 é no máximo o traço de $\mathcal{K}_{0, r}$, portanto finita por hipótese. Procedendo como na demonstração do teorema anterior, deduzimos que

$$
\sum_{n=\beta(m)}^{\infty} n^{2 r+m-1} \lambda_{n^{m}}(\mathcal{K})<\infty
$$

para algum inteiro positivo $\beta(m)$. Repetindo novamente o truque usado na segunda metade da demonstração do Teorema 4.1.6, provamos a convergência anunciada em 4.1.19.

Para encerrar esta seção, gostaríamos de informar que os resultados anteriores podem ser interpretados como versões esféricas de resultados provados em [34, p.120] e [38, 39], onde os núcleos são definidos em produtos cartesianos de intervalos.

\subsection{Otimalidade dos resultados}

Nesta seção, construímos exemplos que mostram que as taxas de decaimento obtidas nos dois últimos resultados da seção anterior são ótimas, ou seja, com as hipóteses consideradas, as taxas não podem ser melhoradas. Começamos com um resultado que garante a otimalidade do decaimento apresentado pelo Teorema 4.1.8. 
Teorema 4.2.1. Seja $\varepsilon>0$ fixado. Se $r \geq 0$ e $m>\varepsilon^{-1}$, então existe um núcleo $L^{2}$-positivo definido com as seguintes propriedades:

(i) $K$ pertence a $W_{2}^{r}$;

(ii) $\mathcal{K}_{0, r}$ é nuclear;

(iii) $\lambda_{n}(\mathcal{K})=o\left(n^{-1-(2 r-1) / m}\right)$;

(iv) Se $\lim _{n \rightarrow \infty} n^{\varepsilon+(m+2 r-1) / m} \lambda_{n}(\mathcal{K})$ existe, então este limite é positivo.

Demonstração: Suponhamos que $\varepsilon m>1$ e que $K$ é o núcleo com expansão

$$
K(x, y) \sim 1+\sum_{n=1}^{\infty} \frac{N(m, n)}{n^{m(1+\varepsilon)+2 r-1}} P_{n}^{m}(x \cdot y), \quad x, y \in S^{m} .
$$

A expansão em harmônicos esféricos do núcleo é facilmente obtida de 4.2.1 com a ajuda da Fórmula da Adição (Teorema 1.4.1)

$$
\sum_{k=1}^{N(m, n)} Y_{n, k}(x) \overline{Y_{n, k}(y)}=N(m, n) P_{n}^{m}(x \cdot y), \quad n=0,1, \ldots, \quad x, y \in S^{m} .
$$

Como

$$
1+\sum_{n=1}^{\infty} \frac{N(m, n)}{n^{m(1+\varepsilon)+2 r-1}} \leq 1+C \sum_{n=1}^{\infty} \frac{1}{n^{2 r+m \varepsilon}}<\infty,
$$

para alguma constante $C$ que depende só de $m$, segue de (1.4.13) que a expansão em 4.2.1) converge uniformemente para $K(x, y)$. Logo, o núcleo $K$ é contínuo e, consequentemente, $K$ é $L^{2}$-positivo definido. O núcleo $K_{0, r}$ tem a seguinte expansão:

$$
K_{0, r}(x, y) \sim \sum_{n=1}^{\infty} \frac{N(m, n) n^{r}(n+m-1)^{r}}{m^{r} n^{m(1+\varepsilon)+2 r-1}} P_{n}^{m}(x \cdot y), \quad x, y \in S^{m} .
$$

Como

$$
\sum_{n=1}^{\infty} \frac{N(m, n) n^{r}(n+m-1)^{r}}{n^{m(1+\varepsilon)+2 r-1}} \leq C_{1} \sum_{n=1}^{\infty} \frac{1}{m^{r} n^{m \varepsilon}}<\infty,
$$

para alguma constante $C_{1}$ dependendo somente de $m$ e $r$, então $K_{0, r}$ é contínuo. Segue que $K \in W_{2}^{r}$. Além disso, usando a ortonormalidade de $Y_{n, k}$, podemos deduzir que

$$
\begin{aligned}
\int_{S^{m}} K_{0, r}(x, x) d \sigma_{m}(x) & =\sum_{n=1}^{\infty} \frac{n^{r}(n+m-1)^{r}}{m^{r} n^{m(1+\varepsilon)+2 r-1}} \sum_{k=1}^{N(m, n)} \int_{S^{m}}\left|Y_{m, n}(x)\right|^{2} d \sigma_{m}(x) \\
& \leq \sigma_{m} C_{1} \sum_{n=1}^{\infty} \frac{1}{n^{m \varepsilon}}<\infty
\end{aligned}
$$

O Teorema 2.3.1 implica que $\mathcal{K}_{0, r}$ é nuclear, de modo que $K$ satisfaz todas as hipóteses exigidas pelo Teorema 4.1.8, ou seja,

$$
\lim _{n \rightarrow \infty} n^{1+(2 r-1) / m} \lambda_{n}(\mathcal{K})=0 .
$$


Finalmente, vamos analisar a taxa de decaimento da sequência $\left\{\lambda_{n}(\mathcal{K})\right\}$. Ela é composta por blocos, o primeiro deles com uma única entrada igual a 1 e o $(n+1)$-ésimo bloco $(n \geq 1) \operatorname{com} N(m, n)$ entradas iguais a $n^{-(m(\varepsilon+1)+2 r-1)}$. Uma rápida análise revela que, para ratificar a taxa de decaimento obtida pelo Teorema 4.1.8, é suficiente verificar que

$$
\lim _{n \rightarrow \infty} \frac{(1+N(m, 1)+\cdots+N(m, n))^{(m+2 r-1) / m}}{n^{m(1+\varepsilon)+2 r-1}}=0 .
$$

Mas, esta igualdade pode ser facilmente verificada, uma vez que, via (4.1.4), podemos majorar o quociente do limite por

$$
\frac{N(m+1, n)^{(m+2 r-1) / m}}{n^{m(1+\varepsilon)+2 r-1}} \leq C_{2} \frac{1}{n^{m \varepsilon}}
$$

para alguma constante positiva $C_{2}$. Para completar a demonstração, supomos que o limite

$$
\lim _{n \rightarrow \infty} n^{\varepsilon+(m+2 r-1) / m} \lambda_{n}(\mathcal{K})
$$

existe e consideramos a subsequência

$$
s_{n}:=\frac{(2+N(m, 1)+\cdots+N(m, n-1))^{\epsilon+(m+2 r-1) / m}}{n^{m(1+\varepsilon)+2 r-1}}
$$

de $\left\{n^{\varepsilon+(m+2 r-1) / m} \lambda_{n}(\mathcal{K})\right\}$. Não é difícil verificar, evocando 4.1.3 e o Lema 4.1.5, que

$$
s_{n} \geq \frac{N(m+1, n-1)^{\epsilon+(m+2 r-1) / m}}{n^{m(1+\varepsilon)+2 r-1}} \geq C_{3} \frac{(n-1)^{m(1+\epsilon)+2 r-1}}{n^{m(1+\varepsilon)+2 r-1}},
$$

para alguma constante positiva $C_{3}$ que depende somente de $m$ e de $r$. Passando ao limite, vemos que

$$
\lim _{n \rightarrow \infty} s_{n} \geq C_{3}>0
$$

o que completa a demonstração.

Para garantir a otimalidade do decaimento encontrado no Teorema 4.1.7, temos o seguinte resultado.

Teorema 4.2.2. Seja $\varepsilon>0$ fixado. Se $m>1 / 2 \varepsilon$ e $r>m / 4$, então existe um núcleo $L^{2}$-positivo definido com as seguintes características:

(i) $K$ pertence a $W_{2}^{r}$;

(ii) $K_{0, r}$ pertence a $L^{2}\left(S^{m} \times S^{m}\right)$;

(iii) $\lambda_{n}(\mathcal{K})=o\left(n^{-1 / 2-(4 r-1) / 2 m}\right)$;

(iv) Se $\lim _{n \rightarrow \infty} n^{\varepsilon+(m+4 r-1) / 2 m} \lambda_{n}(\mathcal{K})$ existe, então este limite é positivo. 
Demonstração: Procedemos como na demonstração do teorema anterior, supondo $\varepsilon m>1 / 2$ e considerando o núcleo $K$ com a expansão

$$
K(x, y) \sim 1+\sum_{n=1}^{\infty} \frac{N(m, n)}{n^{m(\varepsilon+1 / 2)+2 r-1 / 2}} P_{n}^{m}(x \cdot y), \quad x, y \in S^{m} .
$$

Usando 1.4.13) e o Lema 4.1.5, vemos que

$$
\begin{aligned}
\left|\sum_{n=1}^{\infty} \frac{N(m, n)}{n^{m(\varepsilon+1 / 2)+2 r-1 / 2}} P_{n}^{m}(x \cdot y)\right| & \leq \sum_{n=1}^{\infty} \frac{N(m, n)}{n^{m(\varepsilon+1 / 2)+2 r-1 / 2}}\left|P_{n}^{m}(x \cdot y)\right| \\
& \leq C \sum_{n=1}^{\infty} \frac{n^{m-1}}{n^{m(\varepsilon+1 / 2)+2 r-1 / 2}} \\
& \leq C \sum_{n=1}^{\infty} \frac{1}{n^{m(\varepsilon-1 / 2)+2 r+1 / 2}}<\infty,
\end{aligned}
$$

para alguma constante positiva $C=C(m)$, pois $m(\varepsilon-1 / 2)+2 r+1 / 2>1$, por hipótese. Isto garante que a convergência em 4.2.3) é uniforme. Logo, $K$ é contínuo e, consequentemente, $L^{2}$-positivo definido. Para garantir que $K \in W_{2}^{r}$, resta mostrar que $K_{0, r}(\cdot, y) \in L^{2}\left(S^{m}\right)$. Para isto, vamos usar a segunda parte do Teorema 1.3.3. Utilizando o Teorema da Adição, vemos que

$$
\begin{aligned}
K_{0, r}(\cdot, y) & \sim \sum_{n=1}^{\infty} \frac{n^{r}(n+m-1)^{r}}{m^{r} n^{m(\varepsilon+1 / 2)+2 r-1 / 2}} \sum_{k=1}^{N(m, n)} Y_{n, k} \overline{Y_{n, k}(y)} \\
& =\sum_{n=1}^{\infty} \sum_{k=1}^{N(m, n)}\left(\frac{n^{r}(n+m-1)^{r}}{m^{r} n^{m(\varepsilon+1 / 2)+2 r-1 / 2}} Y_{n, k}\right) \overline{Y_{n, k}(y)}
\end{aligned}
$$

Agora, usamos 1.4.12, o Lema 4.1.5 e novamente o Teorema da Adição para provar que

$$
\begin{aligned}
\sum_{n=1}^{\infty} \sum_{k=1}^{N(m, n)}\left|\frac{n^{r}(n+m-1)^{r}}{m^{r} n^{m(\varepsilon+1 / 2)+2 r-1 / 2}} Y_{n, k}\right|^{2} & \leq C_{1} \sum_{n=1}^{\infty} \frac{n^{4 r}}{n^{m(2 \varepsilon+1)+4 r-1}} \sum_{k=1}^{N(m, n)}\left|Y_{n, k}\right|^{2} \\
& \leq C_{2} \sum_{n=1}^{\infty} \frac{n^{m-1}}{n^{m(2 \varepsilon+1)-1}} \\
& =C_{2} \sum_{n=1}^{\infty} \frac{1}{n^{2 m \varepsilon}}<\infty
\end{aligned}
$$

para alguma constante positiva $C_{2}=C_{2}(m, r)$. Assim garantimos que $K_{0, r}(\cdot, y)$ tem quadrado integrável. Como consequência, temos que $K \in W_{2}^{r}$. Resta mostrar que 
$K_{0, r} \in L^{2}\left(S^{m}\right)$ e isto se faz evocando o Teorema 1.3 .24 observando que

$$
\begin{aligned}
\sum_{n=1}^{\infty} \sum_{k=1}^{N(m, n)}\left|\frac{n^{r}(n+m-1)^{r}}{m^{r} n^{m(\varepsilon+1 / 2)+2 r-1 / 2}}\right|^{2} & \leq C_{2} \sum_{n=1}^{\infty} \frac{n^{m-1+4 r}}{n^{m(2 \varepsilon+1)+4 r-1}} \\
& \leq C_{2} \sum_{n=1}^{\infty} \frac{1}{n^{2 m \varepsilon}}<\infty
\end{aligned}
$$

e usando, novamente, o Teorema 1.3.3. Assim, garantimos que $K$ satisfaz todas as hipóteses exigidas pelo Teorema 4.1.7. Logo, seus autovalores devem satisfazer

$$
\lim _{n \rightarrow \infty} n^{1 / 2+(4 r-1) / 2 m} \lambda_{n}(\mathcal{K})=0 .
$$

A seguir, vemos que, realmente, a sequência $\left\{\lambda_{n}(\mathcal{K})\right\}$ possui a taxa de decaimento imposta pelo teorema. Para isto, lembramos que ela é composta por blocos, o primeiro deles com uma única entrada igual a 1 e o $(n+1)$-ésimo bloco $(n \geq 1)$ com $N(m, n)$ entradas iguais a $n^{-(m(\varepsilon+1 / 2)+2 r-1 / 2)}$. Para ratificar a taxa de decaimento obtida pelo Teorema 4.1.7, basta verificar que

$$
\lim _{n \rightarrow \infty} \frac{(1+N(m, 1)+\cdots+N(m, n))^{(m+4 r-1) / 2 m}}{n^{m(\varepsilon+1 / 2)+2 r-1 / 2}}=0,
$$

o que segue lembrando de (4.1.4) e do Lema 4.1.2, e observando que

$$
\begin{aligned}
\frac{(1+N(m, 1)+\cdots+N(m, n))^{(m+4 r-1) / 2 m}}{n^{m(\varepsilon+1 / 2)+2 r-1 / 2}} & =\frac{N(m+1, n)^{(m+4 r-1) / 2 m}}{n^{m(1+\varepsilon)+2 r-1}} \\
& \leq C_{2} \frac{n^{(m+4 r-1) / 2}}{n^{m(1+\varepsilon)+2 r-1}} \\
& =C_{2} \frac{1}{n^{m \varepsilon+(m-1) / 2}},
\end{aligned}
$$

para alguma constante positiva $C_{2}=C_{2}(m, r)$. Completamos a demonstração supondo que existe o limite

$$
\lim _{n \rightarrow \infty} n^{\varepsilon+(m+4 r-1) / 2 m} \lambda_{n}(\mathcal{K})
$$

e considerando a subsequência

$$
s_{n}:=\frac{(2+N(m, 1)+\cdots+N(m, n-1))^{\epsilon+(m+4 r-1) / 2 m}}{n^{m(\varepsilon+1 / 2)+2 r-1 / 2}}
$$

de $\left\{n^{\varepsilon+(m+4 r-1) / 2 m} \lambda_{n}(\mathcal{K})\right\}$. Relembrando 4.1.3 e o Lema 4.1.5, vemos que

$$
s_{n} \geq \frac{N(m+1, n-1)^{\epsilon+(m+4 r-1) / 2 m}}{n^{m(\varepsilon+1 / 2)+2 r-1 / 2}} \geq C_{3} \frac{(n-1)^{m(\epsilon+1 / 2)+2 r-1 / 2)}}{n^{m(\varepsilon+1 / 2)+2 r-1 / 2}},
$$

para alguma constante positiva $C_{3}=C_{3}(m, r)$. Passando ao limite, vemos que

$$
\lim _{n \rightarrow \infty} s_{n} \geq C_{3}>0
$$

completando a demonstração. 


\subsection{Uma família de exemplos}

Esta seção é inteiramente dedicada à apresentação de uma família de núcleos que possuem as características necessárias exigidas pelos Teoremas 4.1.6.4.1.8. Mas, mais importante do que um exemplo satisfazendo estes resultados, é o fato de que todos os principais resultados da tese são empregados para garantir que as hipóteses dos teoremas são realmente satisfeitas, mostrando a funcionalidade destes resultados. Consideramos o conhecido núcleo do calor $K_{t}(t>0)$, uma versão do núcleo gaussiano usual de espaços euclideanos que surge quando se estuda a equação do calor na esfera, como descrito nas referências [47] e [80]. A próxima proposição mostra a existência desta família de núcleos e algumas de suas propriedades. Seu enunciado completo, com outras importantes características destes núcleos, pode ser encontrado em [47] enquanto que uma demonstração está disponível em [80].

Proposição 4.3.1. Seja $m$ um inteiro maior ou igual a 2. Então, existe um núcleo $K \in C^{\infty}\left(\mathbb{R}_{+} \times S^{m} \times S^{m}\right)$ satisfazendo as seguintes propriedade para todo $x, y \in S^{m}$, com $K_{t}(\cdot, \cdot)=K(t, \cdot, \cdot)$, e $t>0$ :

(i) $K_{t}(x, y)=K_{t}(y, x)$;

(ii) $K_{t}$ é positivo definido.

O Teorema 1.3 .27 mostra que $K_{t}$ é $L^{2}$-positivo definido. Uma consequência imediata da propriedade de diferenciabilidade de $K_{t}$ é

$$
K_{t} \in W_{2}^{r}, \quad t>0, \quad r=1,2, \ldots
$$

Além disso, $\mathcal{D}_{y}^{r} K_{t} \in C^{\infty}\left(S^{m} \times S^{m}\right) \subset L^{2}\left(S^{m} \times S^{m}\right), t>0$, e vemos que todas as hipóteses dos Teoremas 4.1.6 e 4.1.7 são satisfeitas.

Para ver que o Teorema 4.1.8 é aplicável, resta somente mostrar que o operador integral gerado por $\mathcal{D}_{y}^{r} K_{t}$ é nuclear para $r \in \mathbb{Z}_{+}$. A compacidade de $S^{m}$ implica na seguinte representação em série ([74, p.89]):

$$
K_{t}(x, y)=\sum_{n=0}^{\infty} e^{-t \lambda_{m, n}} \sum_{k=1}^{N(m, n)} Y_{n, k}(x) \overline{Y_{n, k}(y)}, \quad x, y \in S^{m}
$$

onde $\lambda_{m, n}=n(m-n+1)$. Uma aplicação do Teorema 3.2 .2 revela que a convergência em (4.3.1) é, como desejávamos, uniforme em $S^{m} \times S^{m}$, um fato que corrobora com a exatidão da taxa de decaimento fornecida pelos Teoremas 4.1.6 e 4.1.7. Para seguir em frente, observe que outra aplicação do Teorema 3.2 .2 assegura que

$$
\mathcal{D}_{y}^{r} K_{t}(x, y)=m^{-r} \sum_{n=0}^{\infty} \frac{\lambda_{m, n}^{r}}{e^{t \lambda_{m, n}}} \sum_{k=1}^{N(m, n)} Y_{n, k}(x) \overline{Y_{n, k}(y)},
$$


com convergência uniforme sobre $S^{m} \times S^{m}$. Claramente, este último núcleo é contínuo e positivo definido e, portanto, é $L^{2}$-positivo definido. Mais ainda, a convergência uniforme da série e a ortonormalidade dos harmônicos esféricos garantem que

$$
\begin{aligned}
\int_{S^{m}} \mathcal{D}_{y}^{r} K_{t}(x, x) d \sigma_{m}(x) & =m^{-r} \sum_{n=0}^{\infty} \frac{\lambda_{m, n}^{r}}{e^{t \lambda_{m, n}}} \sum_{k=1}^{N(m, n)} \int_{S^{m}}\left|Y_{n, k}(x)\right|^{2} d \sigma_{m}(x) \\
& =m^{-r} \sum_{n=0}^{\infty} \frac{\lambda_{m, n}^{r}}{e^{t \lambda_{m, n}}} N(m, n)<\infty
\end{aligned}
$$

e o Teorema 2.3.1 implica que $\left(\mathcal{K}_{t}\right)_{0, r}$ é nuclear. 



\section{Referências Bibliográficas}

[1] Axler, S.; Bourdon, P.; Ramey, W., Harmonic Function Theory. Second edition. Graduate Texts in Mathematics, 137. Springer-Verlag, New York, 2001.

[2] Berens, H.; Butzer, P. L.; Pawelke, S., Limitierungsverfahren von Reihen Mehrdimensionaler Kugelfunktionen und deren Saturationsverhalten, Publ. Res. Inst. Math. Sci. Ser. A 4 (1968) 201-268.

[3] Berg, C.; Christensen, J. P. R.; Ressel, P., Harmonic Analysis on Semigroups: Theory of Positive Definite and related functions, Springer-Verlag, 1984.

[4] Brislawn, C., Kernels of trace class operators. Proc. Amer. Math. Soc. 104 (1988), no. 4, 1181-1190.

[5] Brislawn, C., Traceable integral kernels on countably generated measure spaces. Pacific J. Math. 150 (1991), no. 2, 229-240.

[6] Buescu, J., Positive integral operators in unbounded domains, J. Math. Anal. Appl. 296 (2004), no. 1, 244-255.

[7] Buescu, J.; Paixão, A. C., Eigenvalue distribution of Mercer-like kernels, Math. Nachr. 280 (2007), no. 9-10, 984-995.

[8] Buescu, J.; Paixão, A. C., Eigenvalue distribution of positive definite kernels on unbounded domains, Integral Equations Operator Theory, 57 (2007), no. 1, 19-41.

[9] Buescu, J.; Paixão, A. C., Eigenvalues of positive definite integral operators in unbounded intervals, Positivity, 10 (2006), 627-646.

[10] Buescu, J.; Paixão, A. C.; Garcia, F.; Lourtie, I., Positive-definiteness, integral equations and Fourier transforms, J. Integral Equations Appl., 16 (2004), no. 1, $33-52$. 
[11] Carl, B.; Heinrich, S.; Kühn, T., s-numbers of integral operators with Hölder continuous kernels over metric compacta, J. Funct. Anal. 81 (1988), no.1, 54-73.

[12] Carmeli, C.; De Vito, E.; Toigo, A.,Vector valued reproducing kernel Hilbert spaces of integrable functions and Mercer theorem, Anal. Appl. (Singap.), 4 (2006), no. 4, 377-408.

[13] Castro, M. H., Diferentes Noções de Diferenciabilidade para Funções Definidas na Esfera, Dissertação de mestrado, ICMC-USP, 2007 http://www.teses.usp.br/ teses/disponiveis/55/55135/tde-26042007-120351/pt-br.php.

[14] Castro, M. H.; Menegatto, V. A., Eigenvalue decay of positive integral operators on the sphere, submitted for publication.

[15] Castro, M. H.; Menegatto, V. A.; Oliveira, C. P., Laplace-Beltrami differentiability of positive definite kernels on spheres, submitted for publication.

[16] Castro, M. H.; Menegatto, V. A.; Peron, A. P., Integral operators generated by Mercer-like kernels on topological spaces, submitted for publication.

[17] Chang, C.; Ha, C., On eigenvalues of differentiable positive definite kernels, Integral Equations Operator Theory, 33 (1999), 1-7.

[18] Cobos, F.; Kühn, T., Eigenvalues of integral operators with positive definite kernels satisfying integrated Hölder conditions over metric compacta, J. Approx. Theory, 63 (1990), 39-55.

[19] Cochran, J. A.; Lukas, M. A., Differentiable positive definite kernels and Lipschitz continuity, Math. Proc. Camb. Phil. Soc., 104 (1988), 361-369.

[20] Conway, J. B., A Course in Operator Theory. Graduate Studies in Mathematics, 21. American Mathematical Society, Providence, RI, 2000.

[21] Dikmen, C. M.; Reade, J. B., Factorisation of positive definite operators, Arch. Math. (Basel), 91 (2008), no. 4, 339-343.

[22] Dostanić, M. R.; Bilinear expansions of the kernels of some nonselfadjoint integral operators, Mat. Vesnik, 53 (2001), 117-121.

[23] Dostanić, M. R.; Generalization of Mercer theorem for a class of nonselfadjoint operators, Mat. Vesnik, 41 (1989), 22-29. 
[24] Dostanić, M. R.; Generalization of Mercer theorem, Publ. de I'Inst. Math., Nouv. series, 54(68) (1993), 63-70.

[25] Doob, J. L., Measure Theory. Graduate Texts in Mathematics, 143. SpringerVerlag, New York, 1994.

[26] Dudley, R. M.; González-Barrios, J. M., Conditions for integral and other operators to be of trace class. Bol. Soc. Mat. Mexicana (3)4 (1998), no. 1, 105-114.

[27] Ferreira, J. C., Decaimento dos Autovalores de Operadores Integrais Gerados por Núcleos Positivos Definidos, Dissertação de mestrado, ICMC-USP, 2008 http: //www.teses .usp.br/teses/disponiveis/55/55135/tde-01042008-091207/.

[28] Ferreira, J. C.; Menegatto V. A., Eigenvalues of integral operators defined by smooth positive definite kernels, Integral Equations Operator Theory, 64 (2009), no. $1,61-81$.

[29] Ferreira, J. C.; Menegatto, V. A.; Oliveira, C. P., On the nuclearity of integral operators, Positivity, 13 (2009), no. 3, 519-541.

[30] Ferreira, J. C.; Menegatto, V. A.; Peron, A. P., Integral operators on the sphere generated by positive definite smooth kernels, J. Complexity, 24 (2008), no. 5-6, 632-647.

[31] Folland, G. B., Real analysis: Modern Techniques and their Applications, John Wiley \& Sons, 1999.

[32] Fredholm, I., Sur une classe d'équations fonctionnelles, Acta Math., 27 (1903), no 1, 365-390.

[33] Gel'fond, A. O., On estimation of certain determinants and the application of these estimations to the distribution of eigenvalues. Amer. Math. Soc. Transl. (2) 12 1959, 163-179.

[34] Gohberg, I. C.; Krein, M. G., Introduction to the Theory of Linear Nonselfadjoint Operators. Translated from the Russian by A. Feinstein. Translations of Mathematical Monographs, Vol. 18 American Mathematical Society, Providence, R.I., 1969.

[35] Gohberg, I.; Gohberg, S.; Krupnik, N., Traces and Determinants of Linear Operators, Operator Theory: Advances and Applications, 116, Birkhäuser Verlag, Basel, 2000. 
[36] Groemer, H., Geometric Applications of Fourier Series and Spherical Harmonics. (English summary) Encyclopedia of Mathematics and its Applications, 61. Cambridge University Press, Cambridge, 1996.

[37] Grothendieck, A., La théorie de Fredholm. (French) Bull. Soc. Math. France 84 (1956), 319-384.

[38] Ha, C. W., Eigenvalues of differentiable positive definite kernels, SIAM J. Math. Anal., 17 (1986), no. 2, 415-419.

[39] Han, Y. B., Eigenvalues of higher-dimensional positive definite kernels (Chinese), Acta Math. Sinica, 36 (1993) 188-194.

[40] Hille, E.; Tamarkin, J. D., On the characteristic values of linear integral equations. Acta Math. 57 (1931), no. 1, 1-76.

[41] Kadota, T. T., Term by term differentiability of Mercer's expansion, Proc. Amer. Math. Soc., 18 (1967), 69-72.

[42] Kamp, W. P.; Lorentz, R. A.; Rejto, P. A., On a theorem of Stinespring concerning integral operators of trace class. J. Reine Angew. Math. 393 (1989).

[43] König, H., Eigenvalue Distribution of Compact Operators. Operator Theory: Advances and Applications, 16. Birkhäuser Verlag, Basel, 1986.

[44] Kotljar, B. D., Singular numbers of integral operators. (Russian) Differentsial'nye Uravneniya 14 (1978), no. 8, 1473-1477.

[45] Kühn, T., Eigenvalues of integral operators with smooth positive definite kernels, Arch. Math. (Basel), 49 (1987), no. 6, 525-534.

[46] Kühn, T., Eigenvalues of integral operators generated by positive definite Hölder continuous kernels on metric compacta, Indag. Math., 49 (1987), no. 1, 51-61.

[47] Lafferty, J.; Lebanon, G., Diffusion kernels on statistical manifolds. J. Mach. Learn. Res. 6 (2005), 129-163.

[48] Lax, P. D., Functional Analysis. Pure and Applied Mathematics (New York). Wiley-Interscience, New York, 2002.

[49] Lima, E. L., Curso de Análise - Vol 1, 12a edição, Projeto Euclides. Publicação do IMPA, Rio de Janeiro, 2009. 
[50] Lima, E. L., Espaços Métricos, 4a edição, Projeto Euclides. Publicação do IMPA, Rio de Janeiro, 2009.

[51] Lions, J.-L.; Magenes, E., Non-homogeneous Boundary Value Problems and Applications. Vol. I. Translated from the French by P. Kenneth. Die Grundlehren der mathematischen Wissenschaften, Band 181. Springer-Verlag, New YorkHeidelberg, 1972.

[52] Malliavin, P., Integration and Probability. With the collaboration of Hélène Airault, Leslie Kay and Gérard Letac. Graduate Texts in Mathematics, 157. SpringerVerlag, New York, 1995.

[53] Menegatto, V. A.; Peron, A. P.; Oliveira, C. P., Differentiable positive definite kernels on spheres, J. Appl. Anal., 15 (2009), no. 1, 101-117.

[54] Menegatto, V. A.; Piantella, A. C., Old and new on the Laplace-Beltrami derivative, Numer. Funct. Anal. Optim. 32 (2011), no. 3, 309-341.

[55] Mercer, J., Functions of positive and negative type and their connection with the theory of integral equations, Philos. Trans. R. Soc. Lond. Ser. A, 209 (1909), 415-446.

[56] Morimoto, M., Analytic Functionals on the Sphere. Translations of Mathematical Monographs, 178. American Mathematical Society, Providence, RI, 1998.

[57] Munkres, J. R., Topology, a first course, Prentice-Hall, 1975.

[58] Morton, T. M.; Neamtu, M., Error bounds for solving pseudodifferential equations on spheres by collocation with zonal kernels. J. Approx. Theory 114 (2002), no. 2, 242-268.

[59] Novitskiǔ, I. M., Representation of kernels of integral operators by bilinear series (Russian), Sibirsk. Mat. Zh., 25 (1984), no. 5, 114-118.

[60] Novitskiı̌, I. M.; Romanov, M. A., An extension of Mercer's theorem to unbounded operators (Russian), Dalnevostochnii Matematicheskii Sbornik, 7 (1999), 123-132.

[61] Oliveira, C. R., Introdução à Análise Funcional, IMPA, 2007.

[62] Pal'cev, B. V., Asymptotic behavior of the eigenvalues of convolution integral operators on a finite interval with kernels whose Fourier transforms are rational. Soviet Math. Dokl. 11 (1970) 1299-1302. 
[63] Pal'cev, B. V., Asymptotic behavior of the spectrum and eigenfunctions of convolution operators on a finite interval with the kernel having a homogeneous Fourier transform. Soviet Math. Dokl. 218 (1974), no. 5, 1243-1247.

[64] Paraska, V. I., On asymptotics of eigenvalues and singular numbers of linear operators which increase smoothness. (Russian) Mat. Sb. (N.S.) 68 (110) 1965 623-631. (English translation in American Mathematical Society Translations. Series 2, Vol. 79: Thirteen papers on functional analysis and differential equations. American Mathematical Society, Providence, R.I. 1968)

[65] Parfenov, O. G., Estimates of singular values of integral operators with analytic kernels. Vestnik St. Petersburg University Mathematics, vol. 25 (1992), no. 2, pp. 24-32.

[66] Piantella, A. C.; Aproximação na Esfera por Uma Soma com Pesos de Harmônicos Esféricos, Tese de doutorado, ICMC-USP, 2007, http://www.teses.usp.br/ teses/disponiveis/55/55135/tde-08052007-164553/pt-br.php.

[67] Pietsch, A., Eigenvalues and s-numbers. Cambridge Studies in Advanced Mathematics, 13. Cambridge University Press, Cambridge, 1987.

[68] Porter, D.; Stirling, D. S. G., Integral Equations. A Practical Treatment, from Spectral Theory to Applications. Cambridge Texts in Applied Mathematics. Cambridge University Press, Cambridge, 1990.

[69] Reade, J., Eigenvalues of positive definite kernels, SIAM J. Math. Anal., 14 (1983), no. $1,135-140$.

[70] Reade, J., Eigenvalues of positive definite kernels II, SIAM J. Math. Anal., 15 (1984), no. 1, 137-142.

[71] Reade, J., On the sharpness of Weyl's estimate for eigenvalues of smooth kernels, SIAM J. Math. Anal., 16 (1985), no. 3, 137-142.

[72] Reade, J., On the sharpness of Weyl's estimate for eigenvalues of smooth kernels II, SIAM J. Math. Anal., 19 (1988), no. 3, 137-142.

[73] Reimer, M., Multivariate Polynomial Approximation, Vol. 144, Birkhäuser, Berlin, 2003.

[74] Rosenberg, S., The Laplacian on a Riemannian Manifold. An introduction to analysis on manifolds. London Mathematical Society Student Texts, 31. Cambridge University Press, Cambridge, 1997. 
[75] Rudin, W., Uniqueness theory for Laplace series. Trans. Amer. Math. Soc. 68, (1950), 287-303.

[76] Rudin, W., Principles of Mathematical Analysis. Third edition. International Series in Pure and Applied Mathematics. McGraw-Hill Book Co., New YorkAuckland-Düsseldorf, 1976.

[77] Sato, H., Nuclearity of a nonnegative definite integral kernel on a separable metric space. J. Theoret. Probab., 5 (1992), no. 2, 349-353.

[78] Seeley, R. T., Spherical harmonics. Amer. Math. Monthly 731966 \# 4, part II, 115-121.

[79] Sadovinichi $\check{i}$, A. A., Theory of Operators, Consultants Bureau, New York and London, 1991.

[80] Schoen, R.; Yau, S.-T., Lectures on differential geometry. Conference Proceedings and Lecture Notes in Geometry and Topology I. International Press, Cambridge, MA, 1994.

[81] Stinespring, W. F., A sufficient condition for an integral operator to have a trace. J. Reine Angew. Math. 200 (1958) 200-207.

[82] Sun, H., Mercer theorem for RKHS on noncompact sets, J. Complexity, 21 (2005), no. 3, 337-349.

[83] Raman, S. G.; Rao, R. V., Eigenvalues of integral operators on $L^{2}(I)$ given by analytic kernels. (English summary) Integral Equations Operator Theory 18 (1994), no. 1, 109-117.

[84] Young, N., An Introduction to Hilbert Spaces. Cambridge University Press, 1988.

[85] Weyl, H., Das asymptotische Verteilungsgesetz der Eigenwerte linearer partieller Differentialgleichungen (mit einer Anwendung auf die Theorie der Hohlraumstrahlung). (German) Math. Ann. 71 (1912), no. 4, 441-479.

[86] Wehrens, M., Best approximation on the unit sphere in $\mathbb{R}^{k}$. Functional analysis and approximation (Oberwolfach, 1980), pp. 233-245, Internat. Ser. Numer. Math., 60, Birkhäuser, Basel-Boston, Mass., 1981.

[87] Wehrens, M., Legendre-Transformationsmethoden und Approximation von Funktionen auf der Einheitskugel in $R^{3}$. Doctoral Dissertation, RWTH Aachen, 1980. 



\section{Índice Remissivo}

$D^{\alpha}, 13$

$J, 19$

$L^{2}\left(S^{m}\right), 14$

$N(m, k), 13$

$S^{m}, 13$

$T \geq 0,8$

$T^{*}, 7$

$T^{1 / n}, 8$

$T_{\epsilon}^{m}, 17$

$W_{2}^{r}, 17$

$\Delta, 13$

$\Delta_{X}, 21$

$\mathcal{K}, 10$

$\mathbb{R}_{*}^{m+1}, 15$

$\langle f, g\rangle_{2}, 14$

$\lambda_{n}(T), 9$

$\mathcal{H}, 6$

$\mathcal{L}(\mathcal{X}, \mathcal{Y}), \mathcal{L}(X), 6$

$|T|, 8$

$\|\cdot\|_{\mathcal{X}}, 5$

$\sigma_{m}, 13$

$a_{n}=O\left(b_{n}\right), a_{n}=o\left(b_{n}\right), 2$

$d \sigma_{m}, 13$

$s_{n}(T), 9$

$\operatorname{tr}(T), 10$

Aplicação

diferenciável em $S^{m}, 15$ harmônica, 13

homogênea, 13

sequencialmente contínua, 1

Convolução esférica, 19

Critério de Cauchy, 2, 25

Derivada de Laplace-Beltrami, 17

Desigualdade

de Bessel, 6, 26

de Cauchy-Schwarz, 5, 11, 25

de Hölder, 4, 22

de Minkowsky para integrais, 5

Diagonal de um conjunto, 21

Espaço

$\sigma$-finito, 3

$L^{p}, 4$

de harmônicos esféricos, 13

de medida nula, 3

localmente compacto, 2

primeiro enumerável, 1

Extensão radial, 15

Harmônicos esféricos, 13

Identidade de Parseval, 6, 12

Lema da raíz n-ésima, 8

Medida 
$\sigma$-finita, 3

finita, 3

localmente finita, 3

não degenerada, 3

Multi-índice, 13

Núcleo

$L^{2}(X, \mu)$-positivo definido, 12

gerador de um operador integral, 10

hermitiano, 11

positivo definido, 12

suave, 28

Norma

de um operador linear, 7

em $L^{2}\left(S^{m}\right), 14$

euclidiana, 13

Operador

autoadjunto, 7

compacto, 7

de Laplace-Beltrami, 16

de posto finito, 7

diferencial, 13

integral, 10

integral de Laplace-Beltrami, 19

linear, 7

nuclear, 10

positivo, 8

projeção esférica, 17

raíz n-ésima, 8

raíz quadrada, 8

traço de um, 10

translação esférica, 17

Polinômio de Legendre, 15

Produto interno

em $L^{2}\left(S^{m}\right), 14$

usual em $\mathbb{R}^{m+1}, 13$ q.s., 3

Séries numéricas, 3

Teorema

Critério de Cauchy, 2, 25

da adição, 15

da Convergência Dominada, 4

da Imersão de Sobolev, 41

da representação de Riesz, 7

de Dini, 2, 27

de Fubini, 5, 11

de Hilbert-Schmidt, 9

de Mercer, xvii

de Riesz-Fischer, 6, 26

Traço de um operador, 10

Valores singulares, 9 